\title{
Molecular characterization of differences between the tomato immune receptors Fls3 and Fls2
}

Robyn Roberts ${ }^{1}$, Alexander E. Liu ${ }^{1}$, Lingwei Wan ${ }^{1}$, Annie M. Geiger ${ }^{1}$, Sarah R. Hind ${ }^{1}$, Hernan G. Rosli² and Gregory B. Martin ${ }^{1,3^{*}}$

${ }^{1}$ Boyce Thompson Institute for Plant Research, Ithaca, NY 14853, USA

${ }^{2}$ Instituto de Fisiología Vegetal, INFIVE, Universidad Nacional de La Plata, CONICET, La Plata, Buenos Aires, Argentina.

${ }^{2}$ Plant Pathology and Plant-Microbe Biology Section, School of Integrative Plant Science, Cornell University, Ithaca, NY 14853, USA

\section{*Corresponding author:}

G. B. Martin, Boyce Thompson Institute for Plant Research, Ithaca, NY 14853, USA; Tel. 607-2541208; Email: gbm7@cornell.edu

Running title: Molecular differences between Fls3 and Fls2

Keywords: plant immunity; flagellin; PRR-triggered immunity; bacterial speck disease; tomato 


\begin{abstract}
Plants mount defense responses by recognizing indications of pathogen invasion, including microbe-associated molecular patterns (MAMPs). Flagellin from the bacterial pathogen Pseudomonas syringae pv. tomato (Pst) contains two MAMPs, flg22 and flgll-28, that are recognized by tomato receptors Flagellin sensing 2 (Fls2) and Flagellin sensing 3 (Fls3), respectively. It is unknown to what degree each receptor contributes to immunity and if they promote immune responses using the same molecular mechanisms. Characterization of CRISPR/Cas9-generated Fls2 and Fls3 tomato mutants revealed that the two receptors contribute equally to disease resistance both on the leaf surface and in the apoplast. However, striking differences were observed in certain host responses mediated by the two receptors. Compared to Fls2, Fls3 mediated a more sustained production of reactive oxygen species (ROS) and an increase in transcript abundance of 44 tomato genes, with two genes serving as reporters for Fls3. Fls3 had greater in vitro kinase activity and interacted differently with the Pst effector AvrPtoB as compared to Fls2. Using chimeric Fls2/Fls3 proteins, we found that no receptor domain was solely responsible for the Fls3 sustained ROS, suggesting involvement of multiple structural features. This work reveals differences in the immunity outputs between Fls2 and Fls3, suggesting they use distinct molecular mechanisms to activate pattern-triggered immunity in response to flagellin-derived MAMPs.
\end{abstract}




\section{Introduction}

Pseudomonas syringae pv. tomato (Pst) causes bacterial speck disease on tomato, resulting in small, necrotic lesions on leaves, stems, fruit, and flowers (Jones, 1991). The tomato-Pst pathosystem serves as a model for studying bacterial pathogenesis and plant immunity (Oh and Martin, 2011; Pedley and Martin, 2003). The virulence of Pst is primarily determined by a suite of 36 type III effectors which are delivered into the plant cell during the infection process. Two effectors, AvrPto and AvrPtoB, play major roles in interfering with plant immune responses and promote enhanced multiplication of Pst in the leaf apoplast (Buell et al., 2003; Cunnac et al., 2011; Kvitko et al., 2009; Lin and Martin, 2005; Martin, 2012).

Plants detect pathogens by recognizing conserved microbe-associated molecular patterns (MAMPs) via pattern recognition receptor (PRR)-triggered immunity (PTI), or by recognizing pathogen effectors through nucleotide-binding oligomerization domain like receptors (NLR)-triggered immunity (NTI). For Pst, the motility-associated protein flagellin contains two MAMPs, flg22 and flgll-28, that are recognized by the PRRs Flagellin sensing 2 (Fls2) and Flagellin sensing 3 (Fls3), respectively (Gomez-Gomez and Boller, 2000; Hind et al., 2016; Robatzek et al., 2007). Upon recognition of these and other MAMPs, a suite of molecular events occurs to promote defense, including the production of reactive oxygen species (ROS), activation of a mitogen-activated protein kinase (MAPK) cascade, transcriptional reprogramming, callose deposition at the cell wall, stomatal closure, and calcium fluxes (Couto and Zipfel, 2016; Li et al., 2016). While PTI has generally been associated with a moderate inhibition of pathogen growth ( 10 -fold), a recent study shows that flagellin-mediated PTI plays a major role in immunity on the leaf surface for some tomato accessions (decreasing bacterial populations by 150-fold) (Roberts et al., 2019b).

Fls2 and Fls3 bind flg22 and flgll-28 through their extracellular leucine-rich repeat (LRR) domain. In Arabidopsis, upon binding flg22, FLS2 associates with the co-receptor BRI1ASSOCIATED RECEPTOR KINASE (BAK1) and both FLS2 and BAK1 are transphosphorylated to initiate downstream signaling (Couto and Zipfel, 2016; Saijo et al., 2018; Sun et al., 2013). Tomato has two Fls2 genes, Fls2.1 (Solyc02g070890) and Fls2.2 (a paralog of Fls2.1 located 3.8 
kB away from Fls2.1) (Solyc02g070910). Fls2.1 appears to encode the only functional Fls2 in tomato, as a mutation in Fls2.1 causes a complete loss of flg22 recognition (Jacobs et al., 2017). Previous work has shown that not all solanaceous species have Fls3. While tomato, potato, and pepper respond to flgll-28, Nicotiana benthamiana and petunia do not (Cai et al., 2011; Clarke et al., 2013; Hind et al., 2016). A previous study found some similarities between the components involved in Fls2 and Fls3 signaling in tomato, including that Fls3 can interact with Arabidopsis BAK1 upon flgll-28 induction (Hind et al., 2016). Similar to Fls2.1, Fls3 gene expression is induced in leaves after treatment with flg22, flgll-28, or DC3000 $\Delta a v r P t o \Delta a v r P t o B$ (Hind et al., 2016; Rosli et al., 2013). After flgll-28 treatment, there is an increase in the phosphorylation of MAPKs in protoplasts transfected with Fls3 (Hind et al., 2016). N. benthamiana plants silenced for Bak1 have a reduced flgll-28 ROS response compared to control plants, and Fls3 and Arabidopsis BAK1 interact upon flgll-28 treatment when Fls3 and BAK1 are co-expressed in N. benthamiana leaves (Hind et al., 2016). Together, these observations suggest there may be similar factors involved in Arabidopsis Fls2 and tomato Fls3 signaling, but further analysis is needed to determine the molecular mechanisms of Fls3.

Fls2 and Fls3 belong to a family of non-RD kinases due to their lack of conserved arginine (R) and aspartate (D) residues in the activation loop. In Arabidopsis, FLS2 has been shown to have weak autophosphorylation activity that requires the presence of the entire FLS2 intracellular domain, including the inner juxtamembrane domain and the kinase domain (Cao et al., 2013; Gomez-Gomez et al., 2001; Lu et al., 2010; Xiang et al., 2008). Chimeric constructs containing different domains of PRRs have been made to study which domains are important for receptor functions (Albert and Felix, 2010; Albert et al., 2010; Brutus et al., 2010; Hohmann et al., 2018; Holton et al., 2015; Kouzai et al., 2013; Mueller et al., 2012; Wu et al., 2019; Zhou et al., 2019). Chimeric constructs combining the ectodomain of Arabidopsis receptor-like kinase EFR (which detects the bacterial MAMP Ef-Tu) with the intracellular domain of cell wallassociated kinase AtWAK1 (which recognizes oligogalacturonides released from the cell wall) results in a functional chimeric protein that recognizes Ef-Tu and activates plant defenses. However, combining the AtWAK1 ectodomain with the EFR intracellular domain did not result in sufficient defenses, as it did not cause a significant reduction in bacterial growth compared to 
the negative control (Brutus et al., 2010). Combining the FLS2 ectodomain with the EFR intracellular domain also resulted in a functional protein (Brutus et al., 2010). A chimeric crossspecies protein combining the Arabidopsis EFR extracellular domain with the transmembrane and intracellular domains of rice Xa21 (an LRR-RLK that recognizes the sulfated protein RaxX of Xanthamonas oryzae pv. oryzae and encodes resistance) was also functional (Holton et al., 2015). Chimeric constructs swapping Arabidopsis FLS2 and tomato Fls2.1 LRRs aided the authors in finding specific LRR repeats in tomato that are responsible for recognizing the flg15 peptide, which is not recognized by Arabidopsis Fls2 (Mueller et al., 2012; Robatzek et al., 2007).

$\mathrm{NTI}$ is activated upon the recognition of pathogen effectors and causes a suite of molecular events that includes the activation of a MAPK cascade, transcriptional reprogramming, and localized, controlled cell death (hypersensitive response, HR) that is typically associated with a significant inhibition of pathogen growth ( 100-1000 fold). The Pst effectors AvrPto and AvrPtoB are recognized by the cytoplasmic kinase Pto, which acts with the NLR Prf to activate the immune response and results in cell death (Dong et al., 2009; Gutierrez et al., 2010; Lin and Martin, 2007; Martin, 2012; Mathieu et al., 2014; Mucyn et al., 2009; Salmeron et al., 1996; Xing et al., 2007). AvrPto and AvrPtoB are unrelated effectors, but both can bind to Pto. Certain variants of AvrPtoB are also bound by the Fen kinase which is closely related to Pto (Martin, 2012; Martin et al., 1993; Mathieu et al., 2014; Rosebrock et al., 2007). AvrPtoB contains a Ptointeracting domain (PID) near the $\mathrm{N}$-terminus (within residues 1-307), a Fen-interacting domain (FID) in the middle (residues 307-387), and an E3 ubiquitin ligase at the C-terminus. The E3 ligase degrades Fen when Fen is bound to the FID, but because Pto can bind to the FID or PID, Pto can evade E3 ligase degradation by binding to the PID. Deleting the E3 ligase from AvrPtoB (AvrPtoB 1 -387) allows both Pto and Fen to bind the FID, resulting in a strong cell death response (Mathieu et al., 2014). Using a MAPK suppression assay, AvrPtoB ${ }_{1-359}$ was determined to be the minimally biologically functional region of AvrPtoB, which eliminates the E3 ligase and truncates part of the FID but is still able to bind to Fen (Cheng et al., 2011; Martin, 2012; Martin et al., 1993; Mathieu et al., 2014). 
Importantly, NTI may have evolved in response to effectors blocking the PTI pathway to aid pathogen virulence. AvrPtoB binds to the Arabidopsis BAK1 kinase domain (BAK1-KD) and inhibits BAK1 kinase activity in vitro, likely by competing for the BAK1 substrate binding site via an overlapping binding domain with Fen (AvrPtoB $\left.{ }_{250-359}\right)$ and does not require the $\mathrm{E} 3$ ligase domain (Cheng et al., 2011; Rosebrock et al., 2007; Shan et al., 2008). Mutation of AvrPtoB $250-$ 359 to AvrPtoB $_{250-359}(\mathrm{R} 271 \mathrm{~A} / \mathrm{R} 275 \mathrm{~A})$ results in a loss of interaction of AvrPtoB $250-359$ and BAK1-KD by abolishing the interaction of R271 and R275 with the phosphate group of T450 in BAK1, which is required to maintain BAK1 in its active form (Cheng et al., 2011).

Here, we investigated some of the similarities and differences in the immunity outputs between Fls2 and Fls3 in tomato and present data suggesting there are some important differences in the molecular signaling between these two flagellin-sensing receptors in tomato.

\section{Results}

\section{Fls3 and Fls2 each contribute to disease resistance in tomato}

We used the CRISPR/Cas9 system to develop tomato lines that were insensitive to the peptides flgll-28, flg22, or both flgll-28 and flg22. In the background of Rio Grande-prf3 (RG-prf3, which has a mutation in $\operatorname{Prf}$ that eliminates the defense responses associated with the bacterial effectors AvrPto and AvrPtoB), we generated mutations in Fls3 alone ( $\Delta \mathrm{Fls} 3), F / s 2.1$ alone $(\Delta \mathrm{Fls2} .1)$, both Fls2.1 and its paralog Fls2.2 together ( $\Delta \mathrm{Fls2.1/2.2),} \mathrm{and} \mathrm{Fls2.1,} \mathrm{Fls2.2,} \mathrm{and} \mathrm{Fls3}$ together ( $\Delta \mathrm{Fls} 2.1 / 2.2 / 3)$ and verified the presence of mutations using Sanger sequencing (Table S1, Fig. S1a, and Supplementary Methods). The CRISPR/Cas9-generated mutations resulted in a 1 base pair insertion ( $+1 \mathrm{bp}$ ) in the gRNA region of Fls3 for $\Delta \mathrm{Fls3}$, a -7bp deletion (-7bp) in Fls2.1 for $\Delta$ Fls2.1, a -7bp deletion Fls2.1 and a $1 \mathrm{bp}$ insertion (+1bp) in Fls2.2 for $\Delta \mathrm{Fls} 2.1 / 2.2$, and a $+1 \mathrm{bp}$ insertion in each of the three Fls2.1, Fls2.2, and Fls3 genes for $\Delta \mathrm{Fls} 2.1 / 2.2 / 3$. The loss of flgll-28 or flg22 peptide recognition was confirmed in each mutant line using ROS assays (Fig. S1b and Table S1).

To test the contribution of Fls3 and Fls2 to flagellin recognition on the leaf surface and mounting of defense responses, the four mutant lines and wild-type RG-prf3 were dip inoculated with $P$. syringae pv. tomato (Pst) DC3000 strains deleted for avrPto and avrPtoB 


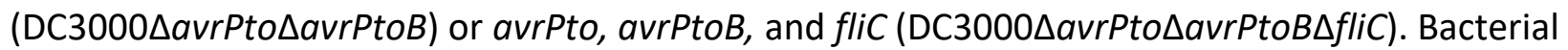
growth in leaves was measured two days after inoculation. In the wild-type, $\Delta$ Fls2.1, $\Delta$ Fls2.1/2.2 and $\Delta$ Fls3 lines we observed significant differences in bacterial growth between the two strains, suggesting that all these lines are able to recognize flagellin. However, in the $\Delta$ Fls $2.1 / 2.2 / 3$ line there was no difference in bacterial growth between DC3000 $\Delta a v r P t o \Delta a v r P t o B$ and DC3000 $a$ avrPto $\Delta a v r P t o B \Delta$ fliC (Fig. 1A).

We next tested whether Fls3 and Fls2 contribute to disease resistance in the leaf apoplast by vacuum infiltrating DC3000 $\Delta a v r P t o \Delta a v r P t o B$ and DC3000 $\Delta a v r P t o \Delta a v r P t o B \Delta$ fliC into $\Delta \mathrm{Fls} 2.1 / 2.2, \Delta \mathrm{Fls} 3$, and $\Delta \mathrm{Fls} 2.1 / 2.2 / 3$ plants and the wild-type line as a control and measuring bacterial growth two days later. Similar to dip-inoculated plants, we observed differences in bacterial growth between the two strains for all of the lines except for $\Delta \mathrm{Fls} 2.1 / 2.2 / 3$. However, the differences in growth observed for the vacuum-inoculated $\Delta \mathrm{Fls} 2.1 / 2.2, \Delta \mathrm{Fls} 3$, and RG-prf3 were much smaller compared to the dip-inoculated plants, with about a 10-fold difference in bacterial growth when vacuum infiltrated versus about a 130-fold difference when dip inoculated (Fig. 1B). This suggests that Fls3 and Fls2 act both on the leaf surface and in the apoplast, but the effect is greater on the leaf surface. We also observed that, compared to RGprf3, speck symptoms were more severe on the plants that are unable to recognize flg22 and/or flgll-28 ( $\Delta \mathrm{Fls} 2.1, \Delta \mathrm{Fls} 2.1 / 2.2, \Delta \mathrm{Fls} 3$, and $\Delta \mathrm{Fls} 2.1 / 2.2 / 3)$. Symptoms were similar on $\Delta \mathrm{Fls} 2.1, \Delta \mathrm{Fls} 2.1 / 2.2$, and $\Delta \mathrm{Fls} 3$ plants, and were slightly more severe on $\Delta \mathrm{Fls} 2.1 / 2.2 / 3$ plants (Fig. 1C). Together, these observations suggest that Fls3 and Fls2 both make important contributions to Pst resistance in tomato.

\section{The ROS response induced by flgll-28 is more sustained compared to that induced by flg22}

We previously observed a more sustained ROS response mediated by Fls3 compared to Fls2 in an accession of the wild species S. pimpinellifolium and hypothesized this might indicate the two PRRs use different molecular mechanisms (Hind et al., 2016). To follow up on this observation, we tested whether tomato (RG-prf3) had a sustained ROS response to flgll-28 compared to flg22. As previously observed, we saw a sustained ROS response for flgll-28 compared to flg22 (Fig. 2A and S2AB). While the amplitude of the flgll-28 and flg22 responses 
was similar, the flgll-28 ROS response continued for up to 100 minutes versus 60 minutes for flg22 (Fig. S2A). To see the generality of this sustained response, we tested another tomato accession (CR293), a tomato heirloom (Galina), and a tomato breeding line (OH05-8144) and found that the flgll-28 response was sustained in all of these lines compared to flg22 (Fig. S2B).

To investigate if this sustained ROS response was specific to tomato, we also tested the flgll-28 ROS response in another solanaceous species, potato (Solanum tuberosum), for which it has been shown that flgII-28 is a major MAMP but not how sustained the associated ROS production was (Clarke et al., 2013; Moroz and Tanaka, 2019). We found that two potato accessions, Snowden and Dakota Crisp, both had a sustained ROS response for flgll-28 compared to flg22, though the amplitude of the response to both MAMPs was similar (Fig. 2B and S2C). A previous study observed a sustained ROS response for pepper (Capsicum annuum cv. 'Jalapeno Early') (Clarke et al., 2013). Because only one variety of eggplant (MM643, Solanum melongena) was previously tested and shown non-responsive to flgll-28 (Clarke et al., 2013), we tested the eggplant variety Shikou for its flgll-28 response and found that it did not respond to flgll-28 (Fig. S3). Together, our data and previous reports suggest that the flgll-28 extended ROS response is conserved in all solanaceous species that respond to flgll-28.

\section{Fls3 specifically regulates expression of a set of defense-related genes in tomato leaves}

Reporter genes are useful for monitoring the activity of specific signaling pathways. From data generated in a previous RNA-Seq analysis of flagellin-induced genes in tomato (Pombo et al., 2017; Rosli et al., 2013) we identified 44 genes whose transcript abundance is significantly increased 6 hours after applying flgll-28, but not flg22 (Table S2). Gene ontology enrichment (GO term) analysis revealed that many of these genes were defense-related, including terms related to proteolysis, peptidase and hydrolase activity, and metabolism (Table S3). Among these genes, the two most highly induced encode a BHLH transcription factor $(B H L H$, Solyc03g114230) and a UDP-glucosyltransferase (UGT, Solyc09g098080). To test whether these genes might serve as specific reporters for Fls3 activity, we used RT-qPCR to estimate gene expression in leaves of the two most highly induced genes, BHLH and UGT, after infiltrating $1 \mu \mathrm{M}$ flgll-28 or flg22, or water as a control. We found that transcript abundance of both $B H L H$ 
and UGT was significantly increased 6 hours after flgll-28 treatment compared to flg22 (Fig. 3).

After flgll-28 treatment, BHLH gene expression was induced by 117-fold and UGT was induced about 303-fold relative to the water treatment. Comparatively, flg22 induced expression of $B H L H$ by 3 -fold and UGT by 12 -fold. These data suggest that BHLH and UGT can serve as specific reporters for the Fls3 response to flgll-28.

\section{Fls3 and Fls2 are inhibited differently by the bacterial effector AvrPtoB}

The effector AvrPtoB blocks PTI by interacting with Bak1 and inhibits Bak1 kinase activity through a mechanism involving the AvrPtoB $250-359$ region (Cheng et al., 2011). We have observed that the Fls3 ROS output is similar between Fls3 natively expressed in tomato and tomato Fls3 that is transiently overexpressed in $N$. benthamiana leaves, making transient expression a useful system to study Fls3 (this study and (Hind et al., 2016)). In Arabidopsis, AvrPtoB $_{1-359}$ binds to the co-receptor BAK1 to inhibit PTI and promote bacterial growth. The mutant, $\operatorname{AvrPtoB}_{1-359}(\mathrm{R} 271 \mathrm{~A} / \mathrm{R} 275 \mathrm{~A})$, is unable to bind to BAK1, and therefore PTI is not suppressed in this pathway. Therefore, if Fls3 and Fls2 both interact with the BAK1 ortholog in tomato (SERK3A/SERK3B), we expect that ROS would be suppressed when Fls3 or Fls2 is coexpressed with AvrPtoB $_{1-359}$, but not with AvrPtoB $_{1-359}(\mathrm{R} 271 \mathrm{~A} / \mathrm{R} 275 \mathrm{~A})$. We tested this by transiently co-expressing Fls3 and Fls2 with AvrPtoB $_{1-359}$ AvrPtoB $_{1-359}(R 271 A / R 275 A)$, or YFP as a control in N. benthamiana that was silenced for Prf using a hairpin construct (hpPrf) (to avoid detection of AvrPtoB by Pto/Prf) and measured ROS after the addition of $100 \mathrm{nM}$ flgll-28 or flg15 peptide, 60 hours after infiltration of the various constructs. We used the flg15 peptide instead of flg22 to circumvent detection of endogenous N. benthamiana Fls2 (flg15 is recognized by tomato Fls2 and not by $N$. benthamiana (Robatzek et al., 2007)). We observed that Fls3 ROS production in response to flgll-28 peptide was similarly inhibited by AvrPtoB 1-359 as the Fls2 ROS response to flg15 (Fig. 4A). We then tested whether co-expressing Fls3 and Fls2 with the mutant AvrPtoB $_{1-359}(R 271 A / R 275 A)$ would fail to suppress PTI in both RLKs, which would lead to a recovery of the Fls3 or Fls2 ROS response. As expected, when Fls2 was coexpressed with AvrPtoB $_{1-359}(\mathrm{R} 271 \mathrm{~A} / \mathrm{R} 275 \mathrm{~A})$ ROS was not inhibited, and we observed similar ROS production as co-infiltrating with the YFP control. Unexpectedly, co-expression of Fls3 with 
AvrPtoB $_{1-359}(\mathrm{R} 271 \mathrm{~A} / \mathrm{R} 275 \mathrm{~A})$ still inhibited Fls3 ROS. Protein expression was confirmed via immunoblot for all proteins except for AvrPtoB $_{1-359}(\mathrm{R} 271 \mathrm{~A} / \mathrm{R} 275 \mathrm{~A})$ (Fig. S4A); however this protein is likely expressed at low levels since it was able to suppress Fls3-associated ROS production in Fig. 4A.

To further investigate whether $\operatorname{AvrPtoB}_{1-359}(\mathrm{R} 271 \mathrm{~A} / \mathrm{R} 275 \mathrm{~A})$ acts differently with Fls2 and Fls3, we tested if bacterial growth was affected. In RG-prf3, DC3000 harboring a plasmid encoding AvrPtoB ${ }_{1-359}(R 271 A / R 275 A)$ (DC3000::AvrPtoB $\left.1-359(R 271 A / R 275 A)\right)$ grew slightly less than the wild type (DC3000::AvrPtoB ${ }_{1-359}$ ) in tomato leaves, though this difference was not statistically significant (Cheng et al., 2011). We vacuum infiltrated DC3000::AvrPtoB 1-359 or DC3000::AvrPtoB 1-359(R271A/R275A) into RG-prf3, $\Delta$ Fls2.1/2.2, $\Delta$ Fls3, or $\Delta$ Fls2.1/2.2/3 plants and measured bacterial populations in leaves 3 days later to test whether Fls3 or Fls2 might interact differently with AvrPtoB when expressed by bacteria. In agreement with the previously published results in (Cheng et al., 2011), we observed a slight, but not statistically significant, reduction in growth of DC3000::AvrPtoB 1 -359(R271A/R275A) compared to DC3000::AvrPtoB 1 -359 in Rio Grande-prf3 (Fig. S4BC). However, in $\Delta$ Fls2.1/2.2 plants the slight difference in growth between the DC3000::AvrPtoB $1-359$ and DC3000::AvrPtoB 1-359(R271A/R275A) was lost, suggesting that PTI is no longer suppressed which allows the DC3000::AvrPtoB 1 ${ }_{359}(\mathrm{R} 271 \mathrm{~A} / \mathrm{R} 275 \mathrm{~A})$ strain to grow more than in wild-type plants. In $\Delta \mathrm{Fls} 3$ plants, a similar difference in bacterial growth of DC3000::AvrPtoB $1-359$ and DC3000::AvrPtoB $1-359(R 271 A / R 275 A)$ was observed as the wild type, suggesting that PTI is not suppressed and growth of DC3000::AvrPtoB ${ }_{1-359}(R 271 A / R 275 A)$ is inhibited. In $\Delta$ Fls2.1/2.2/3 plants, the slight difference in

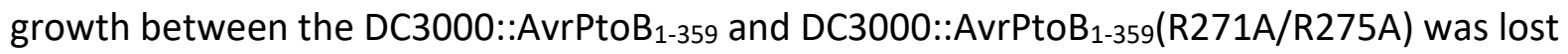
(Fig. S4BC).

Because PTI can have a greater effect on bacterial growth at the leaf surface, we dip inoculated the plants with DC3000::AvrPtoB ${ }_{1-359}$ or DC3000::AvrPtoB 1 -359(R271A/R275A) and measured bacterial growth in leaves to see if the difference in bacterial growth would be greater between the two strains. Although we still did not observe statistically significant differences in bacterial populations by pairwise t-tests in any of the Fls2 or Fls3 mutant or RGprf3, we did observe a similar trend between the vacuum-infiltrated and dip-inoculated plants. 
In the dip-inoculated plants, there was a slight (but not statistically significant) reduction in the bacterial population of DC3000::AvrPtoB 1 -359(R271A/R275A) compared to wild-type DC3000::AvrPtoB 1 -359 in the RG-prf3 and $\Delta$ Fls3 plants, but no reduction was observed in $\Delta \mathrm{Fls} 2.1 / 2.2$ or $\Delta \mathrm{Fls} 2.1 / 2.2 / 3$ plants. We confirmed protein expression of AvrPtoB $1-359$ and AvrPtoB $_{1-359}($ R271A/R275A) in DC3000 via immunoblotting (Fig. S4D). Together, these data suggest that Fls3 and Fls2 interact differently with AvrPtoB in planta.

\section{Fls3 and Fls2 have different kinase activities in vitro}

It is possible that different kinase activities of Fls3 and Fls2 account for their differences in immunity output. It was previously reported that Arabidopsis Fls2 has weak kinase activity in vitro that requires the entire intracellular domain for activity (both the inner juxtamembrane domain and kinase domain) (Cao et al., 2013; Gomez-Gomez et al., 2001; Lu et al., 2010; Xiang et al., 2008). To test whether tomato Fls3 and Fls2 have in vitro kinase activity and if their activity is dependent on the presence of the inner juxtamembrane domain (JM), we made recombinant proteins in BL21 E. coli that contained the Fls3 or Fls2 kinase domain (KD) alone, or the entire cytoplasmic domain containing the JM plus KD (JMKD), expressed in the pDESTHisMBP vector (which has an N-terminal 6xHis-MBP tag), and assayed their kinase activity. As negative controls, we generated variants with substitutions in their ATP binding site, Fls2JMKD(K900Q) and Fls3-JMKD(K877Q), and a control that expressed a short E. coli sequence to allow expression of the His-MBP protein in the PDEST-HisMBP vector. We found that both tomato Fls2 and Fls3 have kinase activity that depends on the presence of the inner juxtamembrane domain (Fig. 5A), and that the in vitro kinase activity for Fls3 is greater than Fls2 (Fig. 5). We added myelin basic protein (MyBP) to the in vitro kinase assays to test whether Fls2 and Fls3 could transphosphorylate this generic substrate. We observed that Fls3, but not Fls2, could transphosphorylate MyBP (Fig. 5B). Because Fls2 and Fls3 both require the inner juxtamembrane domain for in vitro kinase activity, we wanted to know if they required their own cognate inner juxtamembrane domain for activity. We swapped the JM domain between Fls3 and Fls2 (2JM-3KD and 3JM-2KD) and tested them in in vitro kinase assays. We found that swapping the inner juxtamembrane domains between Fls2 and Fls3 completely abolished in 
vitro kinase activity for both proteins (Fig. 5C). These data suggest that Fls3 and Fls2 have differences in their kinase activity that require their corresponding juxtamembrane domain.

\section{Subdomain I contributes to the in vitro differences in kinase activity for Fls3 and Fls2}

We hypothesized that subdomain I of the kinase domain, which is involved in the correct positioning of the ATP substrate in the active site, contributes to the differences in kinase activity we observed between Fls2 and Fls3. We aligned the Fls2 (Fls2.1 and Fls2.2) and Fls3 subdomain I sequences with other immunity-associated tomato kinases and found that Fls2 deviates from the glycine-rich motif (GxGxxG) present in other kinases (Fig. 6A). Fls2 has the conserved, first position glycine, but the second and third glycines are replaced by serines (GxSxxS). Fls3 has the standard GxGxxG motif. We compared the sequence motif of all 42 tomato receptor-like kinases (RLKs) in the Fls2/Fls3 class (class IX-a) (Wei et al., 2015). We aligned the subdomain I kinase domain region of the 42 RLKs to determine the conservation and/or consensus of each of the residues in the GxGxxG motif. Two genes in this class, Solyc10g085110 and Solyc03g118330, likely do not encode active kinases because they lack the subdomain I region and the lysine responsible for binding of the ATP (Fig. S5A). We found that of the 40 RLKs in this class that have a subdomain I, only Fls2 deviates from the GxGxxG motif at the second position glycine (Fig. 6B and Fig. S5A). The third position glycine is more flexible in the class, with 28 RLKs harboring a glycine, 10 RLKs with a serine, 1 RLK with a valine, and 1 RLK with an isoleucine. The first position glycine is conserved in all $40 \mathrm{RLKs}$. To test whether the serines in the Fls2 subdomain I motif impact Fls2 kinase activity, we made substitutions of each serine individually or simultaneously to glycines (GxSxxS to GxGxxS (S881G), GxSxxG (S884G), or GxGxxG (S881G/S884G)). We found that the S881G mutation alone increased the kinase activity of Fls2 (Fig. 6C). In contrast, S884G resulted in a nearly complete loss of kinase activity. However, mutating both residues (S881G/S884G) led to an even stronger increase of kinase activity than S881G alone (Fig. 6C). To see if substituting the glycines to serines in the Fls3 subdomain I motif would impact kinase activity, we mutated the glycines to serines either individually or simultaneously (GxGxxG to GxSxxG (G858S), GxGxxS (G861S), or GxSxxS (G858S/G861S)). We found that the G858S mutation alone caused a near complete loss of in 
vitro kinase activity. The G861S mutation resulted in a strong reduction of kinase activity compared to the wildtype 3JMKD. Mutating both residues (G858S/G861S) also resulted in a reduction of kinase activity that was slightly less than G861S alone (Fig. 6C). These data support the hypothesis that the subdomain I motif contributes to the increased kinase activity of Fls3 compared to Fls2 in vitro.

We next tested whether substitutions in subdomain I of Fls3 and Fls2 affect the ROS output in transient in planta assays in the context of the full-length proteins. We cloned the double mutants Fls2(S881G/S885G) and Fls3(G858S/G861S) into the pGWB417 plant protein expression vector that has a C-terminal myc-tagged protein, cloned the kinase inactive pGWB417::Fls2(K900Q) and pGWB417::Fls3(K877Q) as negative controls, and transiently expressed the proteins in N. benthamiana leaves. ROS responses to $100 \mathrm{nM}$ flgll-28 or flg15 peptide were measured and compared to the wild-type Fls2 or Fls3 proteins. Surprisingly, we found that there was a statistically significant reduction in total ROS production of Fls2(S881G/S885G) compared to wildtype Fls2. There was no statistically significant difference in total ROS production of Fls3(G858S/G861S) compared to wildtype Fls3. We confirmed that all proteins were transiently expressed via immunoblotting (Fig. S5B). These results suggest that although the subdomain I sequence influences in vitro kinase activity, the differences in kinase activity alone do not explain the differences in ROS output between Fls2 and Fls3.

\section{No single domain explains the sustained ROS response of Fls3}

It is also possible that differences in their leucine rich repeat (LRR) domains, which are the extracellular domains that bind the flg22 or flgll-28 peptides, are responsible for the different readouts of these two PRRs. To test this hypothesis, we generated chimeric constructs that swapped the LRR, transmembrane (TM), and kinase domains (KD) between Fls2 and Fls3 for transient expression in $N$. benthamiana. We included the presumed signal peptide and $\mathrm{N}$ terminus within the 'LRR' domain, the inner- and outer-juxtamembrane domains within the 'TM' region, and the C-terminus within the 'KD' (Fig. 7A). We used a low concentration of peptide in the ROS assays (10nM flgll-28 or flg15) to allow detection of subtle differences in the ROS responses of the chimeric constructs. As a control, we also generated the reconstituted 
wild-type constructs, which have the same sequence as the wild-type Fls3 or Fls2 constructs but were generated in the same manner as the chimeric constructs (labeled 3-3-3 or 2-2-2, respectively).

In our transient assays, we found that only the chimeric constructs with the TM and KD from the same receptor responded to peptide (2-3-3 and 3-2-2; Fig. 7B). Specifically, the chimeric construct with the LRR from Fls2 and the TM and KD from Fls3 (2-3-3) responded similarly as the wildtype Fls2 and reconstituted wildtype construct (2-2-2) to the flg15 peptide, both in amplitude and duration of response (Fig. 7B and S6AB). The time at which the maximum ROS response to flg15 peptide occurred was also similar between 2-3-3, Fls2, and 2-2-2 (about 12 minutes after peptide treatment). The normalized ROS responses at the maximum amplitudes were not statistically different between 2-3-3, Fls2, and 2-2-2 (Fig. S6A). Additionally, 45 minutes after peptide treatment there was no significant difference between the ROS responses of 2-3-3, Fls2, and 2-2-2 (Fig. S6B).

When we applied $10 \mathrm{nM}$ of the flgll-28 peptide, only the chimeric construct with the LRR from Fls3 and the TM and KD from Fls2 (3-2-2) activated a ROS response (Fig. 7B). However, while the time at which the maximum response occurred was similar between 3-2-2, wildtype Fls3, and the reconstituted wildtype construct (3-3-3) (about 20 minutes after peptide treatment) and the normalized maximum amplitudes of the ROS responses were not statistically significant (Fig. 7B and Fig. S6A), the ROS response for 3-2-2 was not sustained like Fls3 and 3-3-3 (Fig. 7B and Fig. S6B). At 45 minutes after flgll-28 treatment, the ROS response for 3-2-2 was significantly less than Fls3 and 3-3-3, and was not significantly different from the flgll-28 non-responders (Fig. S6B). All proteins were confirmed to be expressed via immunoblotting, though there were some differences in the levels of expression between the chimeric constructs (Fig. S6C). However, expression of 3-2-2 was similar to Fls3 and 3-3-3, so expression alone does not explain the differences in the sustained ROS responses between Fls3 and 3-3-3 and 3-2-2. Additionally, the expression of 2-3-3 was reduced compared to Fls2 and 22-2 (to levels similar to Fls3 and 3-3-3) but 2-3-3 responded similarly to peptide as Fls2 and 2-22 and this therefore supports that expression levels alone do not account for the differences observed between the chimeric constructs. The data in Fig. 7 and Fig. S6 were normalized to 
the in-plate Fls3 or Fls2 controls to also account for differences in expression between constructs, plants, and technical replicates. Together, these data suggest that no single domain is responsible for the sustained ROS response.

\section{Discussion}

While both Fls2 and Fls3 recognize flagellin-derived MAMPs, their individual contributions to disease resistance was unknown. We used the CRISPR/Cas9 system to generate mutations in the Fls3 and two Fls2 genes in tomato and used them to determine the contributions of the two PRRs to disease resistance. Both Fls3 and Fls2.1 were found to have similar effects on inhibition of Pst growth on the leaf surface and in the leaf apoplast, with the greatest effects occurring on the leaf surface (Fig. 1 and S1). Additionally, we found that a 7 base pair deletion in Fls2.1 alone completely abolished flg22 recognition in tomato, further supporting the idea that Fls2.1 is the only functional receptor for flg22 recognition in tomato leaves as reported previously (Jacobs et al., 2017).

One observation that suggested there may be differences in the molecular mechanisms between Fls2 and Fls3 was that the ROS response to flgll-28 is sustained compared to flg22 (Fig. 2 and S2). We observed this sustained ROS response in both tomato and potato, and data from another study appears to support a sustained ROS response for flgll-28 in pepper (Clarke et al., 2013). While others have reported differences in the amplitude of flgll-28 and flg22 ROS responses in potato (Moroz and Tanaka, 2019), we did not observe these differences under our conditions. This may be attributed to the use of different potato varieties or peptide concentrations in the assays (100nM in our study versus $1 \mu \mathrm{M}$ in (Moroz and Tanaka, 2019)). Additionally, although N. benthamiana does not have an endogenous copy of Fls3, transient overexpression of Fls3 in N. benthamiana also results in a sustained response compared to Fls2 (this study and (Hind et al., 2016)). Previous efforts to express Fls3 in Arabidopsis have been unsuccessful (Hind et al., 2016), so it is currently unknown whether Fls3 can function in species outside the Solanaceae, but future efforts to express Fls3 in more diverse species could determine whether Solanaceous-specific protein partners are involved in the Fls3 ROS response. 
We analyzed RNA-Seq data from previous studies (Pombo et al., 2017; Rosli et al., 2013) and found four genes that were specifically induced upon application of flgll-28 peptide (Fig. 3A). We measured transcript abundance of two of these genes, BHLH and UGT, six hours after treatment of leaves with flgll-28 or flg22 peptide and found that both genes were specifically induced by flgll-28 induction (Fig. 3B). This suggests these two genes are involved in an Fls3specific signaling pathway that is independent of Fls2 and supports the hypothesis that there are differences in the molecular signaling pathways between Fls3 and Fls2. We previously identified three genes whose expression is induced specifically during PTI (Pombo et al., 2014). These genes were not identified as specific Fls3 reporters in the RNA-Seq data indicating they may be induced by both flg22 and flgll-28. While we have yet to find an Fls2-specific reporter gene, future studies using pathway-specific reporter genes may help dissect the differences in the signaling components activated by Fls2 and Fls3.

AvrPtoB inhibits Fls2 by binding to BAK1, and possibly also by directly binding to Fls2 (Cheng et al., 2011; Gohre et al., 2008; Rosebrock et al., 2007; Shan et al., 2008). The R271 and R275 residues in AvrPtoB are important for binding to BAK1, and mutating these residues results in a loss of interaction with BAK1 and a slight reduction in bacterial growth due to the failure of AvrPtoB to suppress PTI (although this reduction is not statistically significant) (Fig. 4 and (Cheng et al., 2011)). We transiently co-expressed Fls2 or Fls3 and AvrPtoB 1 -359 or its variant AvrPtoB $_{1-359}(\mathrm{R} 271 \mathrm{~A} / \mathrm{R} 275 \mathrm{~A})$ in N. benthamiana leaves and found that both Fls2 and Fls3 ROS responses were inhibited upon co-infiltration with AvrPtoB $_{1-359}$ (Fig. 4A). However, when Fls2 and Fls3 were co-infiltrated with AvrPtoB $_{1-359}(R 271 A / R 275 A)$, this AvrPtoB variant still suppressed Fls3 although it did not suppress the Fls2 ROS response and Fls2-driven PTI. These data suggest there may be differences in the molecular interaction between Fls2 and Fls3 and AvrPtoB. Additionally, because AvrPtoB interacts with BAK1 it is possible that Fls2 and Fls3 interact differently with the tomato homologs of BAK1 (SERK3A/SERK3B) and this will be a focus of future studies.

It has been previously reported that Arabidopsis Fls2 has weak autophosphorylation activity in vitro and in vivo that requires the presence of the inner juxtamembrane domain (Cao et al., 2013; Gomez-Gomez et al., 2001; Lu et al., 2010; Xiang et al., 2008). We found that 
tomato Fls2 and Fls3 both have relatively strong autophosphorylation activity in vitro that can be detected after only four hours of exposure to a phosphor-screen. This activity is dependent on the presence of the inner juxtamembrane domain, and it is possible that this may be due to specific important residues within the domain. For example, a previous study examined the requirement of the inner juxtamembrane domain for the rice PRR Xa21 function, and found that the C-terminal region of the inner juxtamembrane domain was required for autophosphorylation (Chen et al., 2010). The authors found a conserved threonine residue (T705) within the juxtamembrane domain at the C-terminal end of the domain that was conserved among plant RLKs, and mutation of this residue (T705A or T705E) resulted in increased susceptibility to Xanthomonas oryzae pv. oryzae. It is still unknown what molecular role this residue and the inner juxtamembrane domain as a whole play in kinase activity, but the authors propose that the threonine may be serving a dual role in receiving and donating a phosphoryl group (Chen et al., 2010). Tomato Fls2 and Fls3 both have this conserved threonine residue, and future research is needed to determine the molecular role of their inner juxtamembrane domains. Some other plant RLK chimeras have been shown to require the cognate inner juxtamembrane domain for their function. For example, a chimeric construct containing the extracellular domain of the rice chitin elicitor receptor CEBiP and the kinase domain from the rice blast resistance protein $\mathrm{Pi}-\mathrm{d} 2$ was only functional if the transmembrane domain originated from Pi-d2 (Kouzai et al., 2013). However, not all RLKs require their cognate inner juxtamembrane domain to function. Swapping the inner juxtamembrane domains of the Arabidopsis RLK CERK1 with BAK1 and Fls2 still resulted in a functional CERK1 protein (Zhou et al., 2019). Our future efforts will focus on understanding why there is a cognate inner juxtamembrane requirement for Fls2 and Fls3.

Subdomain I of the Fls3 kinase domain contributes to the stronger in vitro kinase activity of this protein (Fig. 5,6). A previous study speculated that substituting the second glycine for a serine, as seen in Arabidopsis FLS2 (S879), would lead to a major reduction in kinase activity for FLS2 and supports the data that Arabidopsis has weak autophosphorylation ability (Schwessinger et al., 2011). Therefore, one would predict that changing of S881 to S881G in tomato Fls2 would result in a dramatic increase in kinase activity (Fig. 6C). In fact, when we 
made this substitution we did observe a dramatic increase in kinase activity. However, the S884G substitution resulted in a complete abolishment of kinase activity, yet mutation of both residues (S881G/S884G) caused an even greater increase in kinase activity than S881G alone. Conversely, the G858S substitution in Fls3 caused a complete abolishment of kinase activity, and the G861S and G858S/G861S mutations resulted in a dramatic reduction, but not abolition, of Fls3 kinase activity (Fig. 6C). Further studies are needed to determine why Fls2 requires a serine at residue 884 in the context of GxSxxS or GxGxxS for kinase activity, but these differences between Fls3 and Fls2 suggest there may be differences in the molecular mechanisms of kinase activation between Fls2 and Fls3. Kinase activity alone, however, does not explain the sustained ROS response in planta. When we made the Fls2(S881G/S884G) and Fls3(G858S/G861S) mutations in the context of the full-length protein and overexpressed them in N. benthamiana leaves, the Fls2(S881G/S884G) mutation resulted in a decrease of total ROS production, rather than an increase. For the Fls3(G858S/G861S) mutation, we observed no statistically significant effect of the mutation on total ROS production. It is currently unknown why the in vitro results do not translate to the in planta ROS assays, but future experiments studying the kinase activity in vivo will help uncover the mechanisms of kinase activation in Fls3 and Fls2.

We hypothesized that either the kinase domain or the LRR domain may be responsible for the sustained ROS response observed for Fls3. Therefore, we generated chimeric Fls2 and Fls 3 constructs to test whether either of these domains was solely responsible for the sustained ROS response (Fig. 7 and S6). When we overexpressed the chimeric constructs in $N$. benthamiana leaves and measured the ROS responses to flgll-28 or flg15, we discovered that there is a requirement for the transmembrane domain (TM) and the kinase domain (KD) to be from the same receptor, as only the constructs with a TM and KD originating from the same receptor responded to peptide (Fig. 7 and S6). This observation agrees with the in vitro kinase results that show that kinase activity requires the cognate inner juxtamembrane domain, and suggests that the lack of ROS responses in the chimeric constructs with a TM and KD from different receptors may be due to a lack in kinase activity (Fig. 5C). We also found that neither of the two chimeric constructs that responded to peptide (2-3-3 to flg15 or 3-2-2 to flgll-28) had 
a sustained ROS response (Fig. 7B and S6AB). While the shape of the curve and time at maximum amplitude resembled the receptor matching their LRR domain (12 min for Fls2 and 23-3, and 20 min for Fls3 and 3-2-2), both chimeric constructs were statistically indistinguishable from their negative controls at 45 minutes after peptide treatment, while Fls3 and 3-3-3 maintained the ROS levels at about 50-60\% of their maximum amplitude (Fig. 7 and S6).

Collectively, our data suggest that no single domain is responsible for the sustained ROS response and that multiple structural features unique to Fls3 might contribute to the sustained response. While previous data show that Fls3 has some similar molecular characteristics as Fls2 (Hind et al., 2016), our data suggest that Fls3 acts in a different signaling pathway compared to Fls2 and interacts differently with the bacterial effector AvrPtoB. Determining the different components of the Fls3 and Fls2 pathways and how they differentially interact with Pst effectors may shed light on how Fls3 evolved as a solanaceous-specific flagellin receptor and help us better understand the molecular mechanisms of plant immunity.

\section{Methods}

\section{Plant growth conditions, inoculations, and bacterial growth assays}

Tomato seedlings were grown under the conditions described previously and inoculated as described in (Roberts et al., 2019b) (see the Supplemental Methods for more information). For the dip inoculations, three-week-old seedlings were placed in a $100 \%$ relative humidity chamber for $14 \mathrm{~h}$ prior to inoculation, then dipped into the bacterial suspension of $1 \times 10^{8} \mathrm{CFU}$ $\mathrm{ml}^{-1}$ for 10 seconds. Bacterial populations were quantified on Day 0 and Day 2 or 3 as described previously (Roberts et al., 2019b). See the Supplemental Methods section for more information. For a list of bacterial strains used in this study, see Table S5.

\section{Generation of CRISPR Cas9-mediated knockout lines}

gRNAs were designed to target Fls3, Fls2.1, or both Fls2.1/Fls2.2 as described previously (Jacobs et al., 2017; Zhang et al., 2020) using the tomato genome version SL2.5 (Tomato Genome Consortium, 2012). To induce mutations in both Fls3 and Fls2.1/2.2 in the same plant 
$(\Delta \mathrm{Fls} 2.1 / 2.2 / 3)$, the constructs used to induce the individual mutations were transformed into the Agrobacterium tumefaciens strain LBA4404 and the cultures were mixed 1:1 prior to tomato transformation. Tomato transformations were performed at the Boyce Thompson Institute transformation facility (Gupta and Van Eck, 2016; Van Eck et al., 2019). More information may be found in the Supplemental Methods, Table S1, Table S4-S5, Fig. S1, and on the Plant CRISPR database at plantcrispr.org.

\section{Reactive oxygen species bioassays}

ROS production was measured in response to flgll-28, flg22, or flg15 peptides as described in (Roberts et al., 2019b) using 100nM concentrations for all assays except for the chimeric constructs (10nM flgll-28 and flg15, Fig. 7 and Fig. S6). For Fig. 4 (effector suppression), total ROS accumulation was summed over 45 minutes and the values were normalized to either Fls3 or Fls2 so that all constructs could be directly compared (the samples did not all fit on a single plate). Every experiment was repeated at least three times with four plants per experiment and values represent the means of the four plants, +/- s.d as determined using the Prism 8 program. Shown is one representative replicate for each experiment. See the Supplemental Methods for information about the peptides used in this study.

\section{Reporter genes}

Three, five-week-old Rio Grande-prf3 plants were syringe-infiltrated with $1 \mu \mathrm{M}$ flgll-28 or flg22 peptide or water and sampled $6 \mathrm{~h}$ post infiltration. Biological replicates were taken from each of the three plants infiltrated with peptide or water. RNA extraction, cDNA synthesis, and qRT-PCR was performed as described previously (Pombo et al., 2014), and significance was determined using a pairwise t-test using the Prism 8 program. Primers used for qRT-PCR may be found in Table S4. Gene ontology terms (GO Terms) were determined using the Plant Transcriptional Regulatory Map software (http://plantregmap.cbi.pku.edu.cn/go.php). 


\section{Cloning}

The chimeric constructs were generated via overlap extension PCR. The 2KD, 2JMKD, PCR products were inserted into the entry vector pJLSMART (Mathieu et al., 2014). Chimeric construct ORFs were then recombined into the Gateway vector pGWB417 (Nakagawa et al., 2009; Nakagawa et al., 2007) using the LR Clonase II following the manufacturer's instructions (Thermo Fisher Scientific, https://www.thermofisher.com/us/en/home.html). Mutagenesis of the 2JMKD, 3JMKD, Fls2, and Fls3 clones was performed in the entry vectors using the Q5 SiteDirected Mutagenesis kit following the manufacturer's instructions (New England Biolabs, www.neb.com). For a list of all primers and constructs used in this study, see Table S4 and S5. See the Supplementary Methods for more information.

\section{Agroinfiltrations}

Agroinfiltrations of binary vectors into N. benthamiana were performed as previously described (Hind et al., 2016). All cultures were prepared to a final $\mathrm{OD}_{600}$ of 0.2 . The Fls3- and Fls2- containing bacterial cultures were mixed 1:1 with a construct expressing the $p 19$ viral suppressor of silencing. See the Supplemental Methods for more information.

\section{In vitro kinase assays}

HisMBP-tagged proteins were transformed into BL21 (DE3) pLys Rosetta cells and grown in culture at $37^{\circ} \mathrm{C}$ until the $\mathrm{OD}_{600}$ reached $0.6-0.8$. Protein expression was induced using $1 \mathrm{mM}$ IPTG at $28^{\circ} \mathrm{C}$ for 3-4 h. Cell pellets were suspended in column buffer ( $20 \mathrm{mM}$ Tris- $\mathrm{HCl}, \mathrm{pH} 7.5$, 200 mM NaCl, 1 mM EDTA, 1 mM DTT, 10\% glycerol) supplemented with Complete Easy protease inhibitor cocktail (Millipore Sigma, https://www.sigmaaldrich.com/unitedstates.html), lysed by sonication, mixed with amylose resin (New England BioLabs, https://www.neb.com/), and eluted with $10 \mathrm{mM}$ maltose. In vitro kinase assays were performed using $5 \mu \mathrm{g}$ of each of the various kinase proteins and/or $3 \mu \mathrm{g}$ of myelin basic proteins and conducted as described previously (Roberts et al., 2019a). 


\section{Immunoblotting}

For the transiently-expressed proteins in N. benthamiana, total protein was extracted from Agroinfiltrated leaves, 5-10 $\mu \mathrm{g}$ was run on SDS-PAGE, blotted on PVDF membrane, and detected with anti-Myc antibodies (Genscript, \#A00704 www.genscript.com) and ECL Plus chemiluminescent substrate (Thermo Fisher Scientific, www.thermofisher.com) as described in (Roberts et al., 2019a).

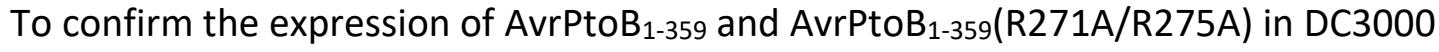
(DC3000::AvrPtoB $1-359$ and DC3000::AvrPtoB $1-359(R 271 A / R 275 A)$, Fig. 4BC and Fig. S4), the hrp promoter on plasmids carrying AvrPtoB $_{1-359}$ or AvrPtoB $_{1-359}(\mathrm{R} 271 \mathrm{~A} / \mathrm{R} 275 \mathrm{~A})$ was induced as described previously (Cheng et al., 2011). Protein was equally loaded on SDS-PAGE for immunoblotting and the HA tag was detected using an anti-HA antibody (Roche, \#11867423001 www.sigmaaldrich.com) and Femto chemiluminescent substrate (Thermo Fisher Scientific, www.thermofisher.com).

\section{Author Contributions}

Conceptualization, R.R. and G.B.M; Methodology, R.R. and G.B.M; Investigation, R.R., A.E.L, L.W., A.M.G., S.R.H, and H.G.R.; Writing-Original Draft, R.R. and G.B.M; Writing-Review and Editing, R.R. and G.B.M.; Funding Acquisition, G.B.M.

\section{Acknowledgments}

We thank Ning Zhang for helpful comments on the manuscript, Jing Zhang, Fabian Giska, and Ning Zhang for performing supporting experiments, Kevin Chen and Ben Carter for experimental assistance, Brian Bell, Jay Miller, and Nick Vail for greenhouse assistance, and Jason Ingram for providing potato tubers used in this study. Funding was provided by National Science Foundation grant IOS-1546625 (GBM). The authors declare no conflicts of interest. 


\section{References}

Albert, M., and Felix, G. (2010). Chimeric receptors of the Arabidopsis thaliana pattern recognition receptors EFR and FLS2. Plant Signal \& Behavior 5:1430-1432.

Albert, M., Jehle, A.K., Mueller, K., Eisele, C., Lipschis, M., and Felix, G. (2010). Arabidopsis thaliana pattern recognition receptors for bacterial elongation factor Tu and flagellin can be combined to form functional chimeric receptors. J Biol Chem 285:19035-19042.

Brutus, A., Sicilia, F., Macone, A., Cervone, F., and De Lorenzo, G. (2010). A domain swap approach reveals a role of the plant wall-associated kinase 1 (WAK1) as a receptor of oligogalacturonides. Proc Natl Acad Sci USA 107:9452-9457.

Buell, C.R., Joardar, V., Lindeberg, M., Selengut, J., Paulsen, I.T., Gwinn, M.L., Dodson, R.J., Deboy, R.T., Durkin, A.S., Kolonay, J.F., et al. (2003). The complete genome sequence of the Arabidopsis and tomato pathogen Pseudomonas syringae pv. tomato DC3000. Proc Natl Acad Sci USA 100:1018110186.

Cai, R.M., Lewis, J., Yan, S.C., Liu, H.J., Clarke, C.R., Campanile, F., Almeida, N.F., Studholme, D.J., Lindeberg, M., Schneider, D., et al. (2011). The plant pathogen Pseudomonas syringae pv. tomato is genetically monomorphic and under strong selection to evade tomato immunity. PLoS Pathog 7:e1002130.

Cao, Y., Aceti, D.J., Sabat, G., Song, J., Makino, S., Fox, B.G., and Bent, A.F. (2013). Mutations in FLS2 Ser938 Dissect Signaling Activation in FLS2-Mediated Arabidopsis Immunity. PLoS Pathog 9:e1003313.

Chen, X., Chern, M., Canlas, P.E., Jiang, C., Ruan, D., Cao, P., and Ronald, P.C. (2010). A conserved threonine residue in the juxtamembrane domain of the XA21 pattern recognition receptor is critical for kinase autophosphorylation and XA21-mediated immunity. J Biol Chem 285:1045410463.

Cheng, W., Munkvold, K.R., Gao, H., Mathieu, J., Schwizer, S., Wang, S., Yan, Y.-b., Wang, J., Martin, G.B., and Chai, J. (2011). Structural Analysis of Pseudomonas syringae AvrPtoB Bound to Host BAK1 Reveals Two Similar Kinase-Interacting Domains in a Type III Effector. Cell Host \& Microbe 10:616-626.

Clarke, C.R., Chinchilla, D., Hind, S.R., Taguchi, F., Miki, R., Ichinose, Y., Martin, G.B., Leman, S., Felix, G., and Vinatzer, B.A. (2013). Allelic variation in two distinct Pseudomonas syringae flagellin epitopes modulates the strength of plant immune responses but not bacterial motility. New Phytol 200:847-860.

Couto, D., and Zipfel, C. (2016). Regulation of pattern recognition receptor signalling in plants. Nat Rev Immunol 16:537-552.

Cunnac, S., Chakravarthy, S., Kvitko, B.H., Russell, A.B., Martin, G.B., and Collmer, A. (2011). Genetic disassembly and combinatorial reassembly identify a minimal functional repertoire of type III effectors in Pseudomonas syringae. Proc Natl Acad Sci USA 108:2975-2980.

Dong, J., Xiao, F., Fan, F., Gu, L., Cang, H., Martin, G.B., and Chai, J. (2009). Crystal structure of the complex between Pseudomonas effector AvrPtoB and the tomato Pto kinase reveals both a shared and a unique interface compared with AvrPto-Pto. Plant Cell 21:1846-1859.

Gohre, V., Spallek, T., Haweker, H., Mersmann, S., Mentzel, T., Boller, T., de Torres, M., Mansfield, J.W., and Robatzek, S. (2008). Plant pattern-recognition receptor FLS2 is directed for degradation by the bacterial ubiquitin ligase AvrPtoB. Curr Biol 18:1824-1832. 
Gomez-Gomez, L., Bauer, Z., and Boller, T. (2001). Both the extracellular leucine-rich repeat domain and the kinase activity of FLS2 are required for flagellin binding and signaling in Arabidopsis. Plant Cell 13:1155-1163.

Gomez-Gomez, L., and Boller, T. (2000). FLS2: an LRR receptor-like kinase involved in the perception of the bacterial elicitor flagellin in Arabidopsis. Mol Cell 5:1003-1011.

Gupta, S., and Van Eck, J. (2016). Modification of plant regeneration medium decreases the time for recovery of Solanum lycopersicum cultivar M82 stable transgenic lines. Plant Cell, Tissue and Organ Culture (PCTOC) 127:417-423.

Gutierrez, J.R., Balmuth, A.L., Ntoukakis, V., Mucyn, T.S., Gimenez-Ibanez, S., Jones, A.M., and Rathjen, J.P. (2010). Prf immune complexes of tomato are oligomeric and contain multiple Pto-like kinases that diversify effector recognition. Plant J 61:507-518.

Hind, S.R., Strickler, S.R., Boyle, P.C., Dunham, D.M., Bao, Z., O'Doherty, I.M., Baccile, J.A., Hoki, J.S., Viox, E.G., Clarke, C.R., et al. (2016). Tomato receptor FLAGELLIN-SENSING 3 binds flgII-28 and activates the plant immune system. Nat Plants 2:16128.

Hohmann, U., Santiago, J., Nicolet, J., Olsson, V., Spiga, F.M., Hothorn, L.A., Butenko, M.A., and Hothorn, M. (2018). Mechanistic basis for the activation of plant membrane receptor kinases by SERKfamily coreceptors. Proc Natl Acad Sci U S A 115:3488-3493.

Holton, N., Nekrasov, V., Ronald, P.C., and Zipfel, C. (2015). The phylogenetically-related pattern recognition receptors EFR and XA21 recruit similar immune signaling components in monocots and dicots. PLoS Pathog 11:e1004602.

Jacobs, T.B., Zhang, N., Patel, D., and Martin, G.B. (2017). Generation of a collection of mutant tomato lines using pooled CRISPR libraries. Plant Physiol 174:2023-2037.

Jones, J.B. (1991). Bacterial speck. In: Compendium of tomato diseases--Jones, J.B., Jones, J.P., Stall, R.E., and Zitter, T.A., eds. St. Paul, MN: APS Press. 26-27.

Kouzai, Y., Kaku, H., Shibuya, N., Minami, E., and Nishizawa, Y. (2013). Expression of the chimeric receptor between the chitin elicitor receptor CEBiP and the receptor-like protein kinase Pi-d2 leads to enhanced responses to the chitin elicitor and disease resistance against Magnaporthe oryzae in rice. Plant Mol Biol 81:287-295.

Kvitko, B.H., Park, D.H., Velasquez, A.C., Wei, C.-F., Russell, A.B., Martin, G.B., Schneider, D.J., and Collmer, A. (2009). Deletions in the repertoire of Pseudomonas syringae pv. tomato DC3000 type III secretion effector genes reveal functional overlap among effectors. PLoS Pathog 5:e1000388.

Li, B., Meng, X., Shan, L., and He, P. (2016). Transcriptional regulation of pattern-triggered immunity in plants. Cell Host \& Microbe 19:641-650.

Lin, N.-C., and Martin, G.B. (2007). Pto- and Prf-mediated recognition of AvrPto and AvrPtoB restricts the ability of diverse Pseudomonas syringae pathovars to infect tomato. Mol Plant-Microbe Interact 20:806-815.

Lin, N.C., and Martin, G.B. (2005). An avrPto/avrPtoB mutant of Pseudomonas syringae pv. tomato DC3000 does not elicit Pto-mediated resistance and is less virulent on tomato. Mol PlantMicrobe Interact 18:43-51.

Lu, D., Wu, S., Gao, X., Zhang, Y., Shan, L., and He, P. (2010). A receptor-like cytoplasmic kinase, BIK1, associates with a flagellin receptor complex to initiate plant innate immunity. Proc Natl Acad Sci USA 107:496-501.

Martin, G.B. (2012). Suppression and activation of the plant immune system by Pseudomonas syringae effectors AvrPto and AvrPtoB. In: Effectors in Plant-Microbe Interactions--Martin, F., and Kamoun, S., eds. Ames, IA: Wiley-Blackwell. 123-154. 
Martin, G.B., Brommonschenkel, S.H., Chunwongse, J., Frary, A., Ganal, M.W., Spivey, R., Wu, T., Earle, E.D., and Tanksley, S.D. (1993). Map-based cloning of a protein kinase gene conferring disease resistance in tomato. Science 262:1432-1436.

Mathieu, J., Schwizer, S., and Martin, G.B. (2014). Pto kinase binds two domains of AvrPtoB and its proximity to the effector E3 ligase determines if it evades degradation and activates plant immunity. PLoS Pathog 10:e1004227.

Moroz, N., and Tanaka, K. (2019). Flgll-28 Is a Major Flagellin-Derived Defense Elicitor in Potato. Mol Plant Microbe Interact:MPMI06190164R.

Mucyn, T.S., Wu, A.J., Balmuth, A.L., Arasteh, J.M., and Rathjen, J.P. (2009). Regulation of tomato Prf by Pto-like protein kinases. Mol Plant-Microbe Interact 22:391-401.

Mueller, K., Bittel, P., Chinchilla, D., Jehle, A.K., Albert, M., Boller, T., and Felix, G. (2012). Chimeric FLS2 receptors reveal the basis for differential flagellin perception in Arabidopsis and tomato. The Plant cell 24:2213-2224.

Nakagawa, T., Ishiguro, S., and Kimura, T. (2009). Gateway vectors for plant transformation. Plant Biotechnology 26:275-284.

Nakagawa, T., Suzuki, T., Murata, S., Nakamura, S., Hino, T., Maeo, K., Tabata, R., Kawai, T., Tanaka, K., Niwa, Y., et al. (2007). Improved Gateway binary vectors: high-performance vectors for creation of fusion constructs in transgenic analysis of plants. Bioscience, biotechnology, and biochemistry 71:2095-2100.

Oh, C.-S., and Martin, G.B. (2011). Effector-triggered immunity mediated by the Pto kinase. Trends in Plant Science 16:132-140.

Pedley, K.F., and Martin, G.B. (2003). Molecular basis of Pto-mediated resistance to bacterial speck disease in tomato. Ann Rev Phytopathol 41:215-243.

Pombo, M.A., Zheng, Y., Fei, Z., Martin, G.B., and Rosli, H.G. (2017). Use of RNA-seq data to identify and validate RT-qPCR reference genes for studying the tomato-Pseudomonas pathosystem. Sci Rep 7:44905.

Pombo, M.A., Zheng, Y., Fernandez-Pozo, N., Dunham, D.M., Fei, Z., and Martin, G.B. (2014). Transcriptomic analysis reveals tomato genes whose expression is induced specifically during effector-triggered immunity and identifies the Epk1 protein kinase which is required for the host response to three bacterial effector proteins. Genome Biol 15:492.

Robatzek, S., Bittel, P., Chinchilla, D., Kochner, P., Felix, G., Shiu, S.H., and Boller, T. (2007). Molecular identification and characterization of the tomato flagellin receptor LeFLS2, an orthologue of Arabidopsis FLS2 exhibiting characteristically different perception specificities. Plant Mol Biol 64:539-547.

Roberts, R., Hind, S.R., Pedley, K.F., Diner, B.A., Szarzanowicz, M.J., Luciano-Rosario, D., Majhi, B.B., Popov, G., Sessa, G., Oh, C.S., et al. (2019a). Mai1 protein acts between host recognition of pathogen effectors and MAPK signaling. Mol Plant Microbe Interact 32:1496-1507.

Roberts, R., Mainiero, S., Powell, A.F., Liu, A.E., Shi, K., Hind, S.R., Strickler, S.R., Collmer, A., and Martin, G.B. (2019b). Natural variation for unusual host responses and flagellin-mediated immunity against Pseudomonas syringae in genetically diverse tomato accessions. New Phytol 223:447461.

Rosebrock, T.R., Zeng, L., Brady, J.J., Abramovitch, R.B., Xiao, F., and Martin, G.B. (2007). A bacterial E3 ubiquitin ligase targets a host protein kinase to disrupt plant immunity. Nature 448:370-374.

Rosli, H.G., Zheng, Y., Pombo, M.A., Zhong, S., Bombarely, A., Fei, Z., Collmer, A., and Martin, G.B. (2013). Transcriptomics-based screen for genes induced by flagellin and repressed by pathogen effectors identifies a cell wall-associated kinase involved in plant immunity. Genome Biol 14:R139. 
Saijo, Y., Loo, E.P., and Yasuda, S. (2018). Pattern recognition receptors and signaling in plant-microbe interactions. Plant J 93:592-613.

Salmeron, J.M., Oldroyd, G.E., Rommens, C.M., Scofield, S.R., Kim, H.S., Lavelle, D.T., Dahlbeck, D., and Staskawicz, B.J. (1996). Tomato Prf is a member of the leucine-rich repeat class of plant disease resistance genes and lies embedded within the Pto kinase gene cluster. Cell 86:123-133.

Schwessinger, B., Roux, M., Kadota, Y., Ntoukakis, V., Sklenar, J., Jones, A., and Zipfel, C. (2011). Phosphorylation-dependent differential regulation of plant growth, cell death, and innate immunity by the regulatory receptor-like kinase BAK1. PLoS Genet 7:e1002046.

Shan, L., He, P., Li, J., Heese, A., Peck, S.C., Nuernberger, T., Martin, G.B., and Sheen, J. (2008). Bacterial effectors target the common signaling partner BAK1 to disrupt multiple MAMP receptorsignaling complexes and impede plant immunity. Cell Host \& Microbe 4:17-27.

Sun, Y., Li, L., Macho, A.P., Han, Z., Hu, Z., Zipfel, C., Zhou, J.M., and Chai, J. (2013). Structural basis for flg22-induced activation of the Arabidopsis FLS2-BAK1 immune complex. Science 342:624-628.

Tomato Genome Consortium. (2012). The tomato genome sequence provides insights into fleshy fruit evolution. Nature 485:635-641.

Van Eck, J., Keen, P., and Tjahjadi, M. (2019). Agrobacterium tumefaciens-Mediated Transformation of Tomato. Methods Mol Biol 1864:225-234.

Wei, Z., Wang, J., Yang, S., and Song, Y. (2015). Identification and expression analysis of the LRR-RLK gene family in tomato (Solanum lycopersicum) Heinz 1706. Genome 58:121-134.

Wu, J., Reca, I.B., Spinelli, F., Lironi, D., De Lorenzo, G., Poltronieri, P., Cervone, F., Joosten, M., Ferrari, S., and Brutus, A. (2019). An EFR-Cf-9 chimera confers enhanced resistance to bacterial pathogens by SOBIR1- and BAK1-dependent recognition of elf18. Mol Plant Pathol 20:751-764.

Xiang, T., Zong, N., Zou, Y., Wu, Y., Zhang, J., Xing, W., Li, Y., Tang, X., Zhu, L., Chai, J., et al. (2008). Pseudomonas syringae effector AvrPto blocks innate immunity by targeting receptor kinases. Curr Biol 18:74-80.

Xing, W., Zou, Y., Liu, Q., Liu, J., Luo, X., Huang, Q., Chen, S., Zhu, L., Bi, R., Hao, Q., et al. (2007). The structural basis for activation of plant immunity by bacterial effector protein AvrPto. Nature 449:243-247.

Zhang, N., Roberts, H.M., Van Eck, J., and Martin, G.B. (2020). Generation and molecular characterization of CRISPR/Cas9-induced mutations in 63 immunity-associated genes in tomato reveals specificity and a range of gene modifications. Frontiers in Plant Science In Press.

Zhou, Q., Liu, J., Wang, J., Chen, S., Chen, L., Wang, J., Wang, H.B., and Liu, B. (2019). The juxtamembrane domains of Arabidopsis CERK1, BAK1, and FLS2 play a conserved role in chitininduced signaling. J Integr Plant Biol.

Figure legends

Figure 1. Both Fls2 and Fls3 contribute to disease resistance on the leaf surface and in the leaf apoplast in tomato. Bacterial populations in tomato leaves in CRISPR/Cas9-generated mutants

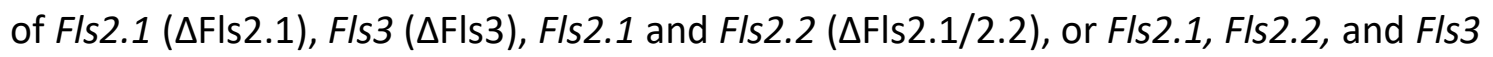
( $\Delta$ Fls2.1/2.2/3) or Rio Grande-prf3 (RG-prf3) were measured 0 and 2 days after (A) dipinoculating plants in bacterial suspensions $\left(1 \times 10^{8} \mathrm{cfu} / \mathrm{mL}\right)$, or $(B)$ vacuum infiltrating plants with bacterial suspensions $\left(1 \times 10^{4} \mathrm{cfu} / \mathrm{mL}\right)$. Shown are the means of three individual plants 
indicated as separate points, and horizontal lines are the means of the three plants +/- s.d.. Statistical significance was determined by pairwise t-test, where ${ }^{*} P<0.05,{ }^{* *} P<0.01$, or $P>0.05$ (not significant, n.s.). (C) Photos of CRISPR/Cas9-generated mutant plants vacuum infiltrated with DC3000 $\triangle$ avrPto $\triangle a v r P t o B$ at $1 \times 10^{4} \mathrm{CFU} / \mathrm{mL}$, taken 1 week post inoculation. Experiments were repeated three times with similar results and data are representative of a single replicate. See also Figure S1 and Tables S1-S2.

Figure 2. The flgll-28 ROS response is sustained in both tomato and potato. Oxidative burst produced over 45 minutes in response to $100 \mathrm{nM}$ flgll-28 or flg22 peptide or water in (A) tomato variety Rio Grande-prf3, or (B) potato variety Snowden. ROS was measured in relative light units (RLU) and results shown are means +/- s.d. ( $n=4$ for tomato, $n=3$ for potato) and are representative of three independent experiments. See also Figures S2 and S3.

Figure 3. Expression of four tomato genes is induced by the Fls3 pathway but not the Fls2 pathway. qRT-PCR of the two most highly induced genes (BHLH transcription factor (BHLH, Solyc03g114230) and a UDP-glucosyltransferase (UGT, Solyc09g098080)) was performed $6 \mathrm{hr}$ after infiltrating $1 \mu \mathrm{M}$ flg22 or flgll-28 peptide or water, and normalized to an internal control (ARD2). Data shown are the average fold change in expression compared to the water control of three individual plants from a single experimental replicate shown as separate points, and horizontal lines are the means of the three plants +/- s.d.. Significance was determined by pairwise t-test and $P$ values are shown above each pairwise comparison. Asterisks represent significance of $* P<0.05$ or $* * P<0.01$. Results are representative of three independent experiments.

Figure 4. Fls3 and Fls2 are inhibited differently by the bacterial effector AvrPtoB. (A) Fls3 or Fls2 or their kinase inactive variants ( $\mathrm{K} 877 \mathrm{Q}$ or $\mathrm{K} 900 \mathrm{Q}$, respectively) were co-infiltrated with YFP or the AvrPtoB variants (AvrPtoB ${ }_{1-359}$ or $A_{v r P t o B}-359$ (R271A/R275A) into N. benthamiana leaves expressing a hairpin construct to knock down expression of $\operatorname{Prf}$ (hpPrf). Constructs were overexpressed under a 35S promoter. Samples were taken for ROS assays 48 hours post infiltration, and data shown are the means of four individual plants indicated as separate points, and horizontal lines are the means of the three plants +/- s.d.. Means are the total ROS values in response to $100 \mathrm{nM}$ flg15 (Fls2) or flgll-28 (Fls3) peptide, accumulated over 45 minutes and normalized to the mean of Fls3 or Fls2 (100\% ROS) to allow direct comparison of the Fls3 and Fls2 ROS values. Data are representative of four independent experiments. Statistical significance was determined using two-way ANOVA with Tukey's multiple comparisons post-test with statistical significance cutoff $P<0.05$. (B) Bacterial populations in tomato leaves of mutants of Fls2.1 and Fls2.2 ( $\Delta \mathrm{Fls} 2.1 / 2.2$ ), Fls3 ( $\Delta \mathrm{Fls} 3)$, or Fls2.1, Fls2.2, and Fls3 ( $\Delta$ Fls2.1/2.2/3) or Rio Grande-prf3 (RG-prf3) were measured 0 and 3 days after dip- 
inoculating plants with bacterial suspensions $\left(1 \times 10^{8} \mathrm{cfu} / \mathrm{mL}\right)$ using a DC3000 strain expressing AvrPtoB $_{1-359}$ (labeled AvrPtoB $_{1-359}$ ) or its variant AvrPtoB $_{1-359}(R 271 A / R 275 A$ ) (labeled $\mathrm{R} 271 \mathrm{~A} / \mathrm{R} 275 \mathrm{~A})$. Shown are the means of three individual plants indicated as separate points, and horizontal lines are the means of the three plants +/-s.d.. There were no statistically significant differences using a pairwise t-test in bacterial growth between the two strains for each plant $(P>0.05)$. (C) Table showing the averages and standard deviations (SD) of the data shown in (B) on Day 3. Data are from a single experiment and are representative of two replicates. See also Figure S4.

Figure 5. Fls3 and Fls2 vary in their kinase activity in vitro. The kinase domain alone (KD) or inner juxtamembrane domain plus the kinase domain (JMKD) of Fls3 and Fls2 were expressed as recombinant proteins in the PDEST-HisMBP vector and tested for in vitro kinase activity. The kinase inactive mutants for Fls3(K877Q) and Fls2(K900Q) were generated in the background of the JMKD constructs. HisMBP protein was used as a negative control. (A) Fls3 or Fls2 kinase domains (KD) or kinase domains plus the inner juxtamembrane domains (JMKD) were tested in in vitro kinase assays. (B) Myelin basic protein (MyBP) was added to the in vitro kinase assays to test the ability of the kinases to transphosphorylate the MyBP substrate. (C) Kinase assays swapping the inner juxtamembrane domains of Fls3 and Fls2 to test the requirement for their cognate inner juxtamembrane domain. 3JM-2KD expresses the inner juxtamembrane domain from Fls3 and the kinase domain from Fls2. 2JM-3KD expresses the inner juxtamembrane domain from Fls2 and the kinase domain from Fls3. Data shown are representative of at least three independent experiments. Below each panel is the exposure length to the phosphorscreen (4 or 14 hours) and the SDS-PAGE gel percentage.

Figure 6. Subdomain I contributes to the differences in kinase activity between Fls 3 and Fls 2 in vitro. (A) Subdomain I in tomato showing the GxGxxG motif. In red is the lysine involved in ATP binding. Highlighted in yellow are the conserved glycines in the motif, and in blue are the deviant serines in Fls2. (B) MEME LOGO showing the prevalence of the GxGxxG motif in class IXa of the tomato RLKs, which includes Fls2 and Fls3. Red arrows indicate the three glycines in the GxGxxG motif. Only Fls2 has a different residue in the second position glycine (red asterisk). (C) In vitro kinase activity of Fls2JMKD and Fls3JMKD containing mutations in subdomain I. Data are representative of three independent replicates. (D) Total ROS accumulated over 45 minutes after applying $100 \mathrm{nM}$ flg15 (Fls2 proteins) or flgll-28 peptide (Fls3 proteins) on leaf discs collected after transient expression of the various proteins in N. benthamiana. Protein overexpression was driven by the CaMV 355 promoter. Shown are the means of four individual plants indicated as separate points, and horizontal lines are the means of the four plants +/s.d.. Data are from a single experiment and are representative of three independent replicates. 
Significance was determined via ANOVA followed by Tukey's post-test with a significance cutoff of $P<0.05$. See also Figure S5.

Figure 7. No single domain governs the sustained ROS response. (A) Schematic diagram showing the design of the chimeric Fls2 and Fls3 constructs. Amino acid numbers corresponding to the domains used to generate the chimeric proteins are shown above each schematic. Leucine rich repeat, LRR; transmembrane domain, TM; and kinase domain, KD. The labels for each chimeric construct are as follows: the ' 2 ' or ' 3 ' indicates whether the domain came from Fls2 (' 2 ') or Fls3 (' 3 '). The first number indicates the LRR, second number indicates the TM, and third number indicates the KD. The reconstituted Fls2 (2-2-2) and Fls3 (3-3-3) were included as controls and are identical in sequence to wildtype Fls2 or Fls3, respectively. (B) ROS curves comparing the chimeric constructs for the sustained response. Constructs were transiently expressed in $N$. benthamiana leaves under control of the CaMV 35 S promoter and ROS activity was measured over 45 minutes after the addition of $10 \mathrm{nM}$ flg15 or flgll-28 peptide. Data are the means of four plants $(n=4)$, and error bars represent $+/$ - s.d. and are representative of three independent experiments. The two chimeric constructs that responded to peptide are highlighted in blue. The unadulterated controls are highlighted in red, and the reconstituted controls are highlighted in gold. See also Figure S6. 
Dip inoculation

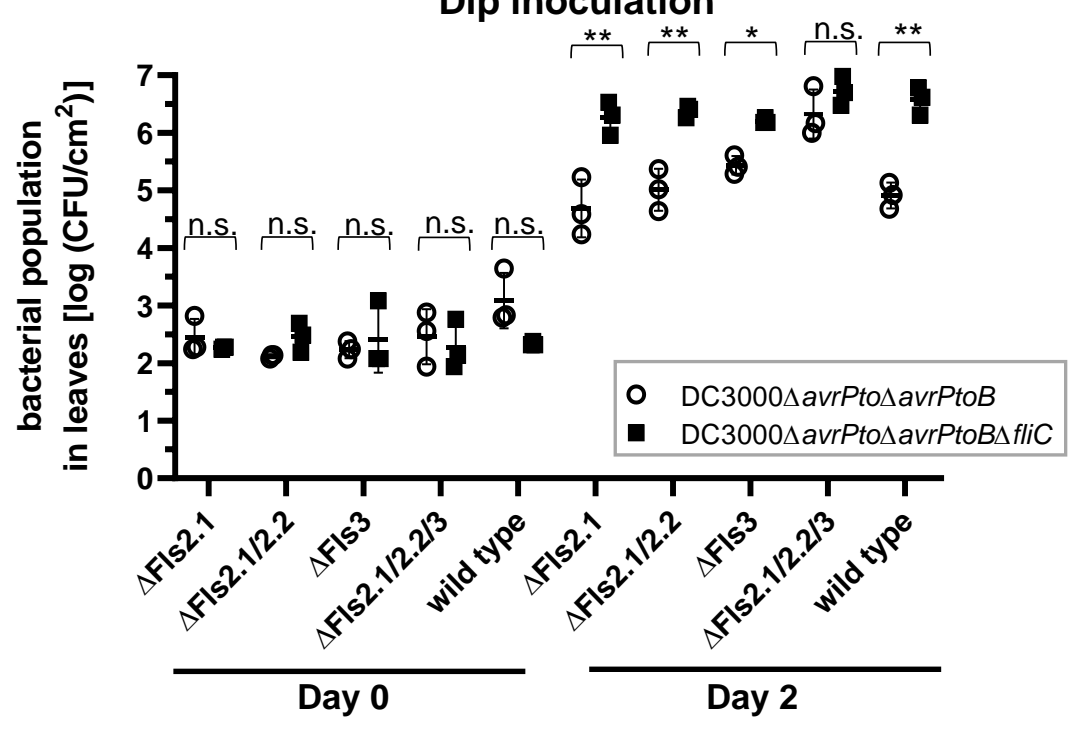

B

\section{Vacuum infiltration}

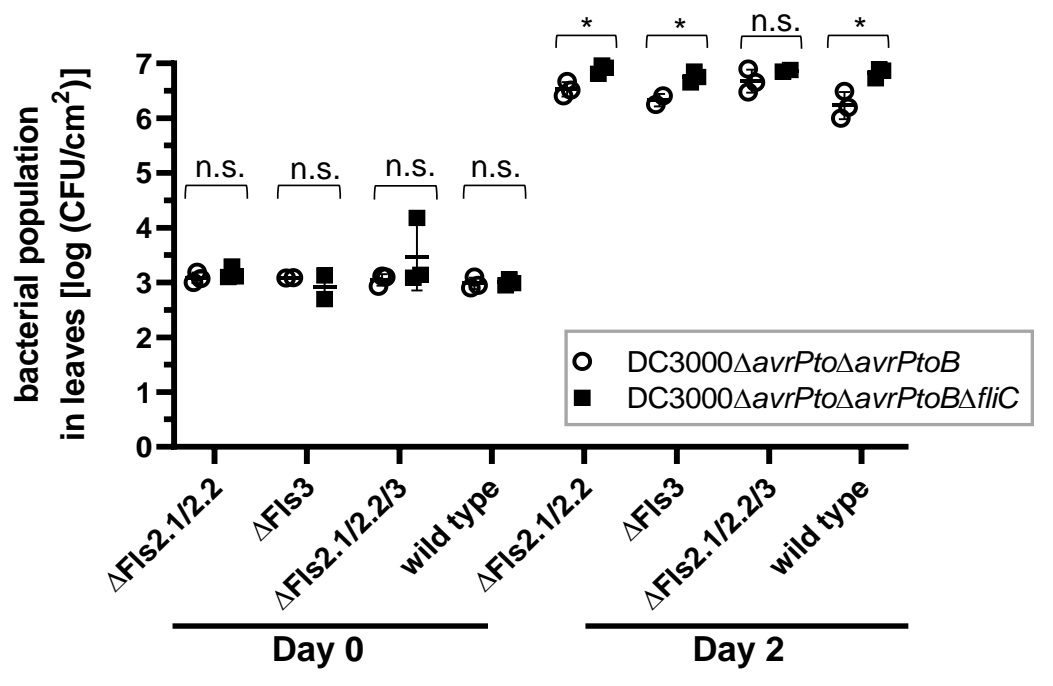

\section{C}

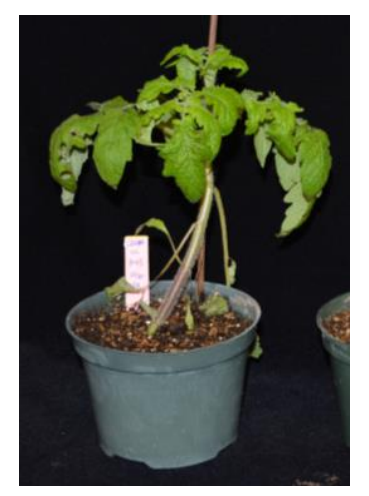

Rio Grande-prf3

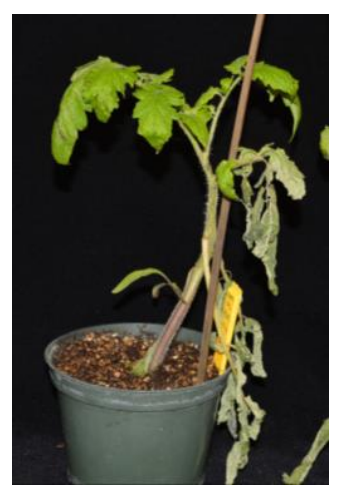

$\Delta$ FIs2.1

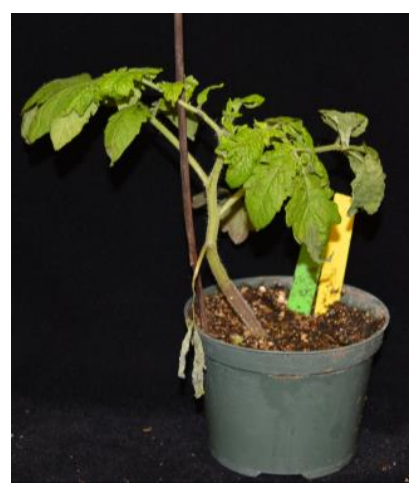

$\Delta$ Fls3

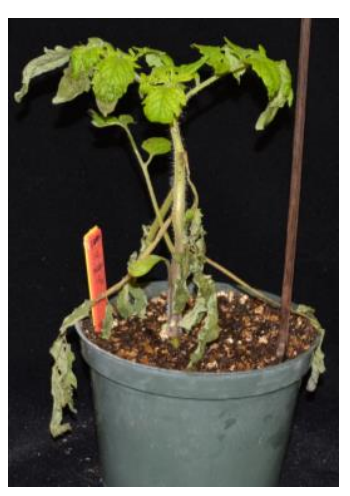

$\Delta \mathrm{FIs} 2.1 / 2.2$

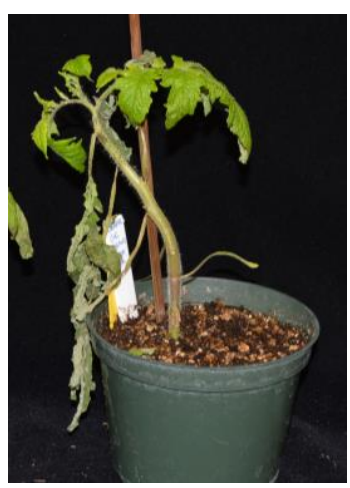

$\Delta \mathrm{FIs} 2.1 / 2.2 / 3$

Figure 1. Both Fls2 and FIs3 contribute to disease resistance on the leaf surface and in the leaf apoplast in tomato. Bacterial populations in tomato leaves in CRISPR/Cas9-generated mutants of Fls2.1 ( $\Delta \mathrm{Fls} 2.1), F l s 3(\Delta \mathrm{Fls} 3), F l s 2.1$ and Fls2.2 ( $\Delta \mathrm{Fls} 2.1 / 2.2)$, or Fls2.1, Fls2.2, and Fls3 ( $\Delta \mathrm{Fls} 2.1 / 2.2 / 3)$ or Rio Grande-prf3 (RG-prf3) were measured 0 and 2 days after (A) dip-inoculating plants in bacterial suspensions $\left(1 \times 10^{8} \mathrm{cfu} / \mathrm{mL}\right)$, or $(B)$ vacuum infiltrating plants with bacterial suspensions $\left(1 \times 10^{4} \mathrm{cfu} / \mathrm{mL}\right)$. Shown are the means of three individual plants indicated as separate points, and horizontal lines are the means of the three plants + /- s.d. Statistical significance was determined by pairwise t-test, where ${ }^{*} P<0.05$, ${ }^{* *} P<0.01$, or $P>0.05$ (not significant, n.s.). (C) Photos of CRISPR/Cas9-generated mutant plants vacuum infiltrated with DC3000 2 avrPto $\triangle a v r P t o B$ at $1 \times 10^{4} \mathrm{CFU} / \mathrm{mL}$, taken 1 week post inoculation. Experiments were repeated three times with similar results and data are representative of a single replicate. See also Figure S1 and 


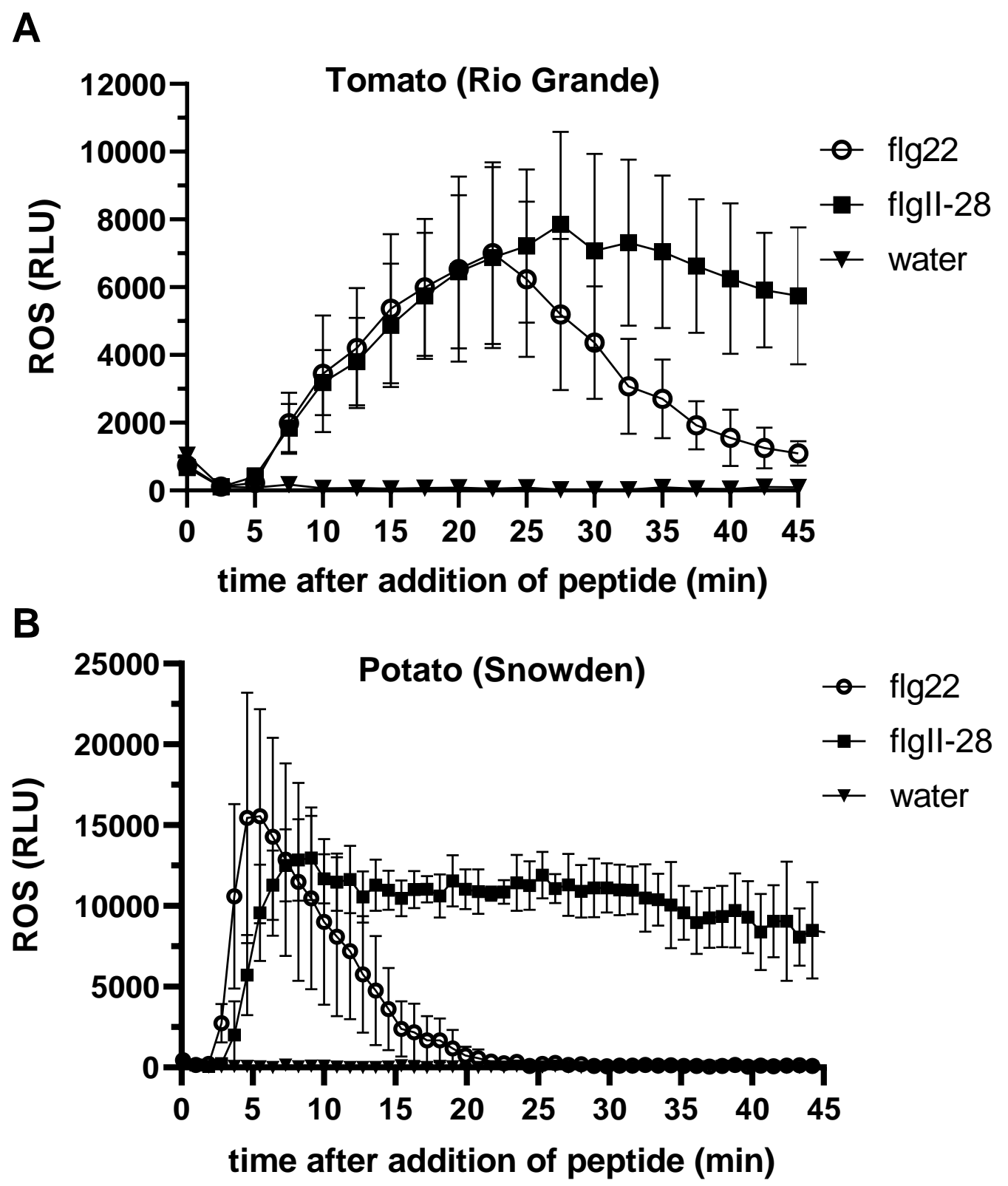

Figure 2. The flgll-28 ROS response is sustained in both tomato and potato.

Oxidative burst produced over 45 minutes in response to $100 \mathrm{nM}$ flgll-28 or flg22 peptide or water in (A) tomato variety Rio Grande-prf3, or (B) potato variety Snowden. ROS was measured in relative light units (RLU) and results shown are means +/- s.d. ( $n=4$ for tomato, $n=3$ for potato) and are representative of three independent experiments. See also Figures S2 and S3. 


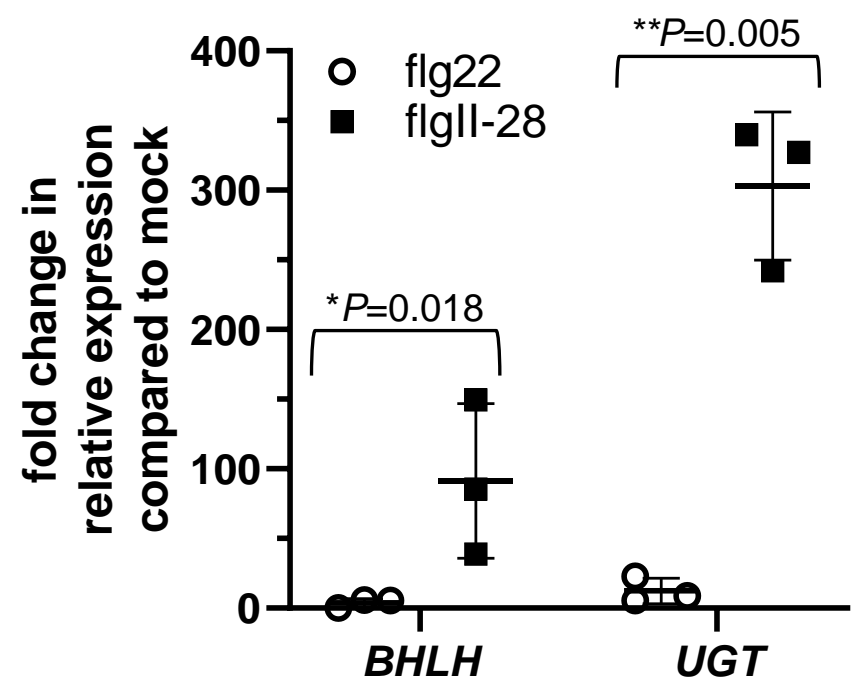

reporter gene

Figure 3. Expression of four tomato genes is induced by the Fls3 pathway but not the Fls2 pathway. qRT-PCR of the two most highly induced genes (BHLH transcription factor (BHLH, Solyc03g114230) and a UDP-glucosyltransferase (UGT, Solyc09g098080)) was performed $6 \mathrm{hr}$ after infiltrating $1 \mu \mathrm{M}$ flg22 or flgll-28 peptide or water, and normalized to an internal control (ARD2). Data shown are the average fold change in expression compared to the water control of three individual plants from a single experimental replicate shown as separate points, and horizontal lines are the means of the three plants $+/-$ s.d.. Significance was determined by pairwise t-test and $P$ values are shown above each pairwise comparison. Asterisks represent significance of ${ }^{*} P<0.05$ or ${ }^{* *} P<0.01$. Results are representative of three independent experiments. 
Figure 4. Fls3 and Fls2 are inhibited differently by the bacterial effector AvrPtoB. (A) Fls3 or Fls2 or their kinase inactive variants (K877Q or K900Q, respectively) were co-infiltrated with YFP or the AvrPtoB variants $\left(\right.$ AvrPtoB $_{1-359}$ or AvrPtoB $_{1-359}(\mathrm{R} 271 \mathrm{~A} / \mathrm{R} 275 \mathrm{~A})$ into N. benthamiana leaves expressing a hairpin construct to knock down expression of Prf (hpPrf). Constructs were overexpressed under a $35 S$ promoter. Samples were taken for ROS assays 48 hours post infiltration, and data shown are the means of four individual plants indicated as separate points, and horizontal lines are the means of the three plants +/- s.d.. Means are the total ROS values in response to $100 \mathrm{nM}$ flg15 (Fls2) or flgll-28 (Fls3) peptide, accumulated over 45 minutes and normalized to the mean of Fls3 or Fls2 (100\% ROS) to allow direct comparison of the Fls3 and Fls2 ROS values. Data are representative of four independent experiments. Statistical significance was determined using two-way ANOVA with Tukey's multiple comparisons post-test with statistical significance cutoff $P<0.05$. (B) Bacterial populations in tomato leaves of mutants of Fls2.1 and Fls2.2 ( $\Delta \mathrm{Fls} 2.1 / 2.2), F l s 3(\Delta \mathrm{Fls} 3)$, or Fls2.1, Fls2.2, and Fls3 $(\Delta \mathrm{Fls} 2.1 / 2.2 / 3)$ or Rio Grande-prf3 (RG-prf3) were measured 0 and 3 days after dip-inoculating plants with bacterial suspensions $\left(1 \times 10^{8} \mathrm{cfu} / \mathrm{mL}\right)$ using a DC3000 strain expressing AvrPtoB 1-359 $_{\text {(labeled }}$ AvrPtoB $_{1-359}$ ) or its variant AvrPtoB $_{1-359}(R 271 A / R 275 A)$ (labeled R271A/R275A). Shown are the means of three individual plants indicated as separate points, and horizontal lines are the means of the three plants +/- s.d.. There were no statistically significant differences using a pairwise t-test in bacterial growth between the two strains for each plant ( $P>0.05)$. (C) Table showing the averages and standard deviations (SD) of the data shown in (B) on Day 3. Data are from a single experiment and are representative of two replicates. See also Figure 54. 
bioRxiv preprint doi: https://doi.org/10.1101/2020.02.04.934133; this version posted February 5 , 2020. The copyright holder for this preprint

A (which was not certified bu peefrey/ \&whighe author/funder, who has granted bioRxiv a license to display the preprint in perpetuita. It is made
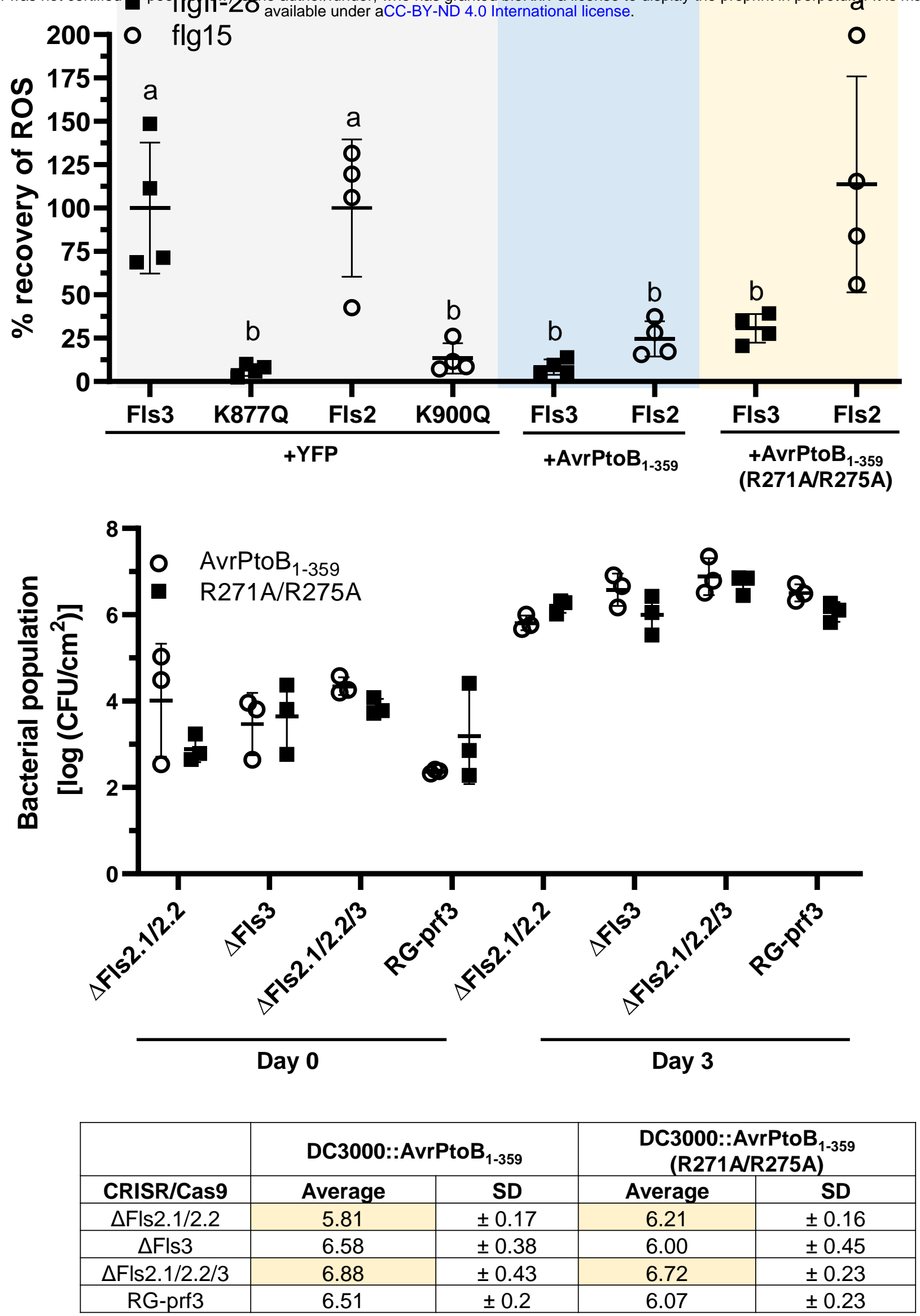

Figure 4. Fls3 and FIs2 are inhibited differently by the bacterial effector AvrPtoB. Figure legend on previous page. See also Figure S4. 


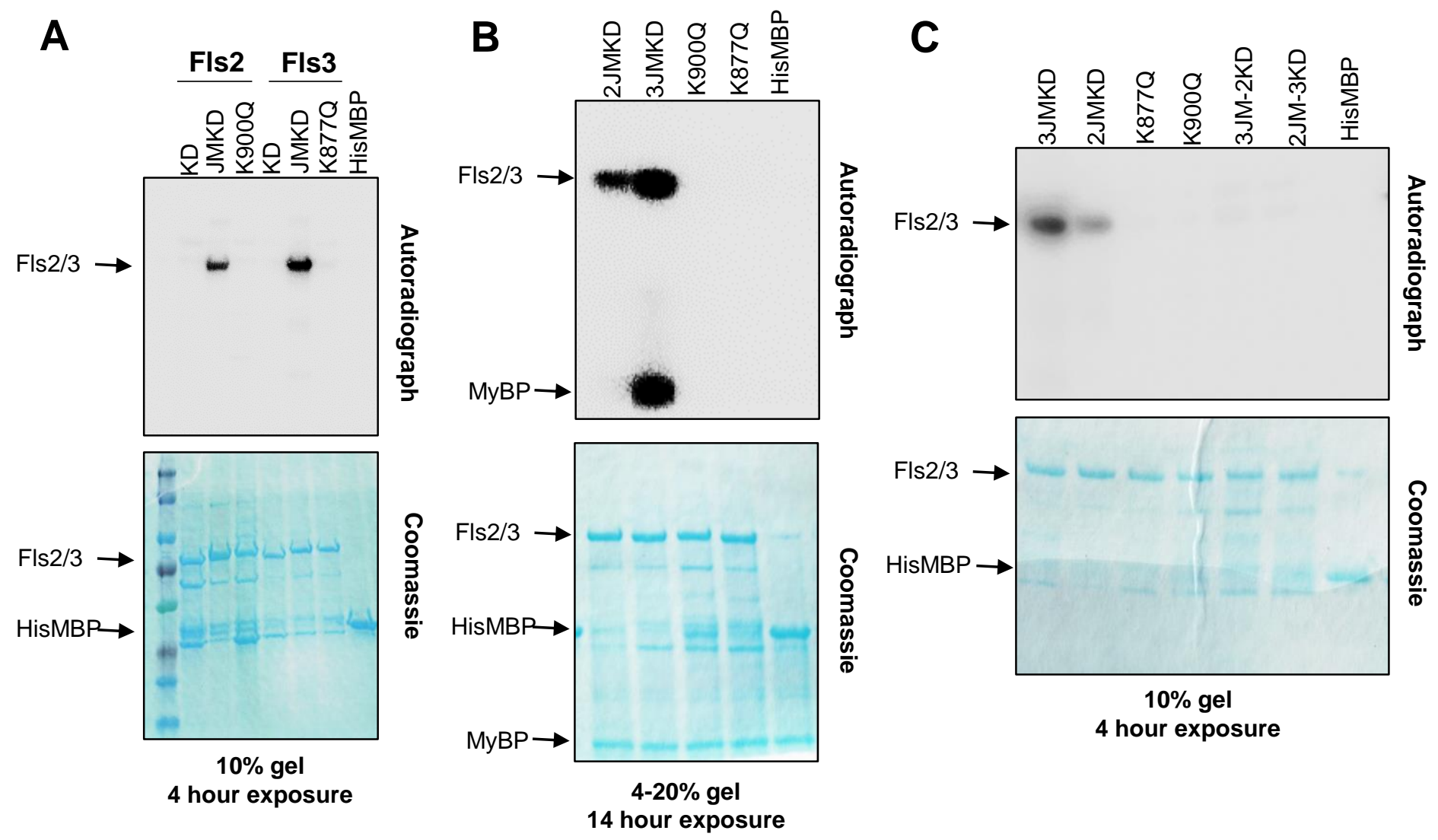

Figure 5. Fls3 and Fls2 vary in their kinase activity in vitro. The kinase domain alone (KD) or inner juxtamembrane domain plus the kinase domain (JMKD) of Fls3 and Fls2 were expressed as recombinant proteins in the PDEST-HisMBP vector and tested for in vitro kinase activity. The kinase inactive mutants for Fls3(K877Q) and Fls2(K900Q) were generated in the background of the JMKD constructs. HisMBP protein was used as a negative control. (A) Fls3 or Fls2 kinase domains (KD) or kinase domains plus the inner juxtamembrane domains (JMKD) were tested in in vitro kinase assays. (B) Myelin basic protein (MyBP) was added to the in vitro kinase assays to test the ability of the kinases to transphosphorylate the MyBP substrate. (C) Kinase assays swapping the inner juxtamembrane domains of Fls3 and Fls2 to test the requirement for their cognate inner juxtamembrane domain. 3JM-2KD expresses the inner juxtamembrane domain from Fls3 and the kinase domain from Fls2. 2JM-3KD expresses the inner juxtamembrane domain from Fls2 and the kinase domain from Fls3. Data shown are representative of at least three independent experiments. Below each panel is the exposure length to the phosphor-screen (4 or 14 hours) and the SDS-PAGE gel percentage. 
bioRxiv preprint doi: https://doi.org/10.1101/2020.02.04.934133; this version posted February 5, 2020. The copyright holder for this preprint (which was not certified by peer review) is the author/funder, who has granted bioRxiv a license to display the prep int in perpetuity. It is made available under aCC-BY-ND 4. Bnternational license.

SIFls2.1 NIIGASSLSTVYKGTLE-DGKIVAVK SIFls2.2 NIIGASSSSNVYKGTLE-GGKIVAVK Fls3 NLIGVGGSGSVYKATLA-SGIVVAIK SlWak1 RILGRGGNGIVYKGILS-DNRIVAIK Bti9 NKIGQGGFGAVYYAELR-GEKA-AIK CORE

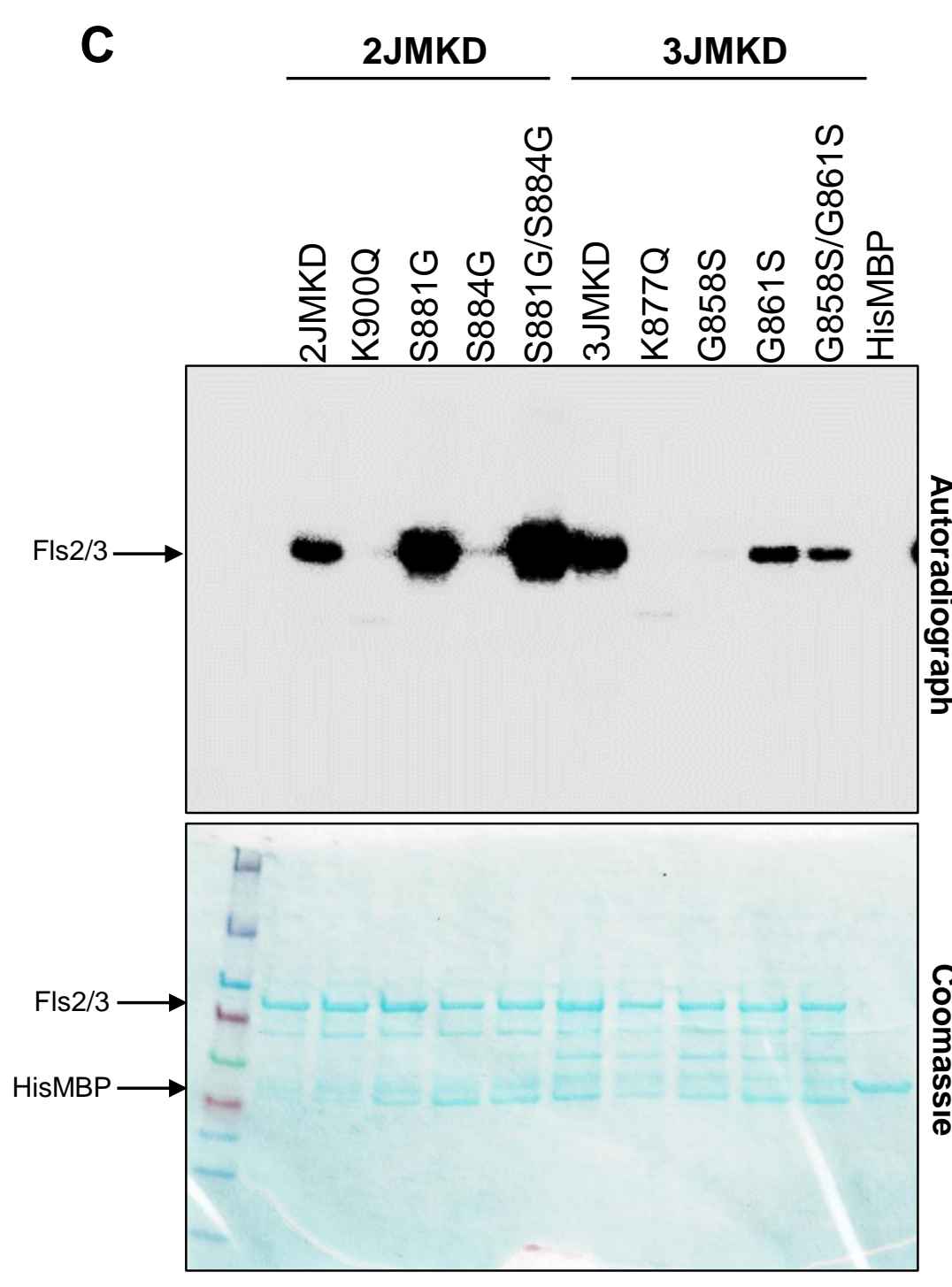

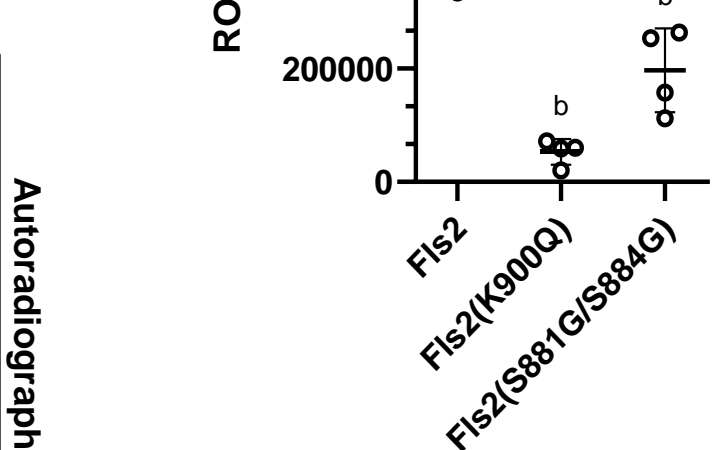

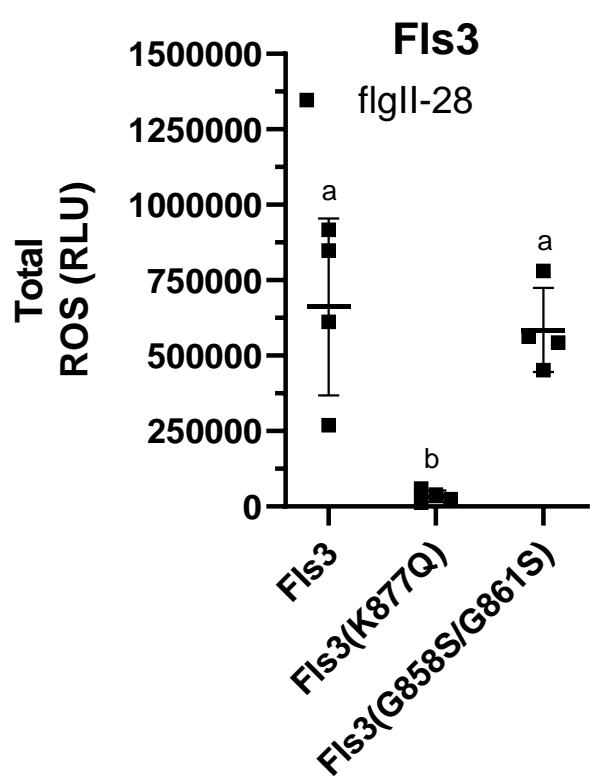

Figure 6. Subdomain I contributes to the differences in kinase activity between Fls3 and Fls2 in vitro. (A) Subdomain I in tomato showing the GxGxxG motif. In red is the lysine involved in ATP binding. Highlighted in yellow are the conserved glycines in the motif, and in blue are the deviant serines in Fls2. (B) MEME LOGO showing the prevalence of the GxGxxG motif in class IXa of the tomato RLKs, which includes Fls2 and Fls3. Red arrows indicate the three glycines in the GxGxxG motif. Only Fls2 has a different residue in the second position glycine (red asterisk). (C) In vitro kinase activity of FIs2JMKD and FIs3JMKD containing mutations in subdomain I. Data are representative of three independent replicates. (D) Total ROS accumulated over 45 minutes after applying $100 \mathrm{nM}$ flg15 (Fls2 proteins) or flgll-28 peptide (Fls3 proteins) on leaf discs collected after transient expression of the various proteins in $N$. benthamiana. Protein overexpression was driven by the CaMV 35S promoter. Shown are the means of four individual plants indicated as separate points, and horizontal lines are the means of the four plants +/- s.d.. Data are from a single experiment and are representative of three independent replicates. Significance was determined via ANOVA followed by Tukey's post-test with a significance cutoff of $P<0.05$. See also Figure S5. 


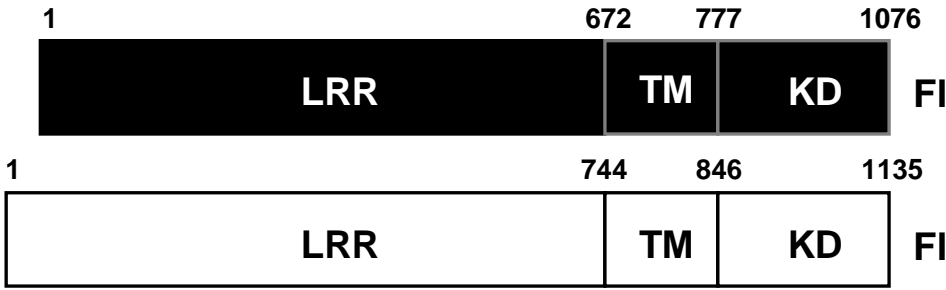

Fls3/3-3-3

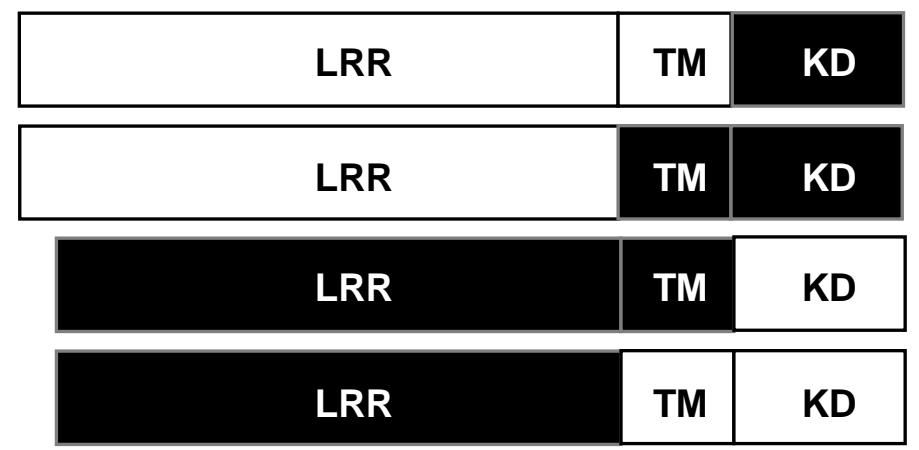

3-3-2

3-2-2

2-2-3

2-3-3

\section{B}
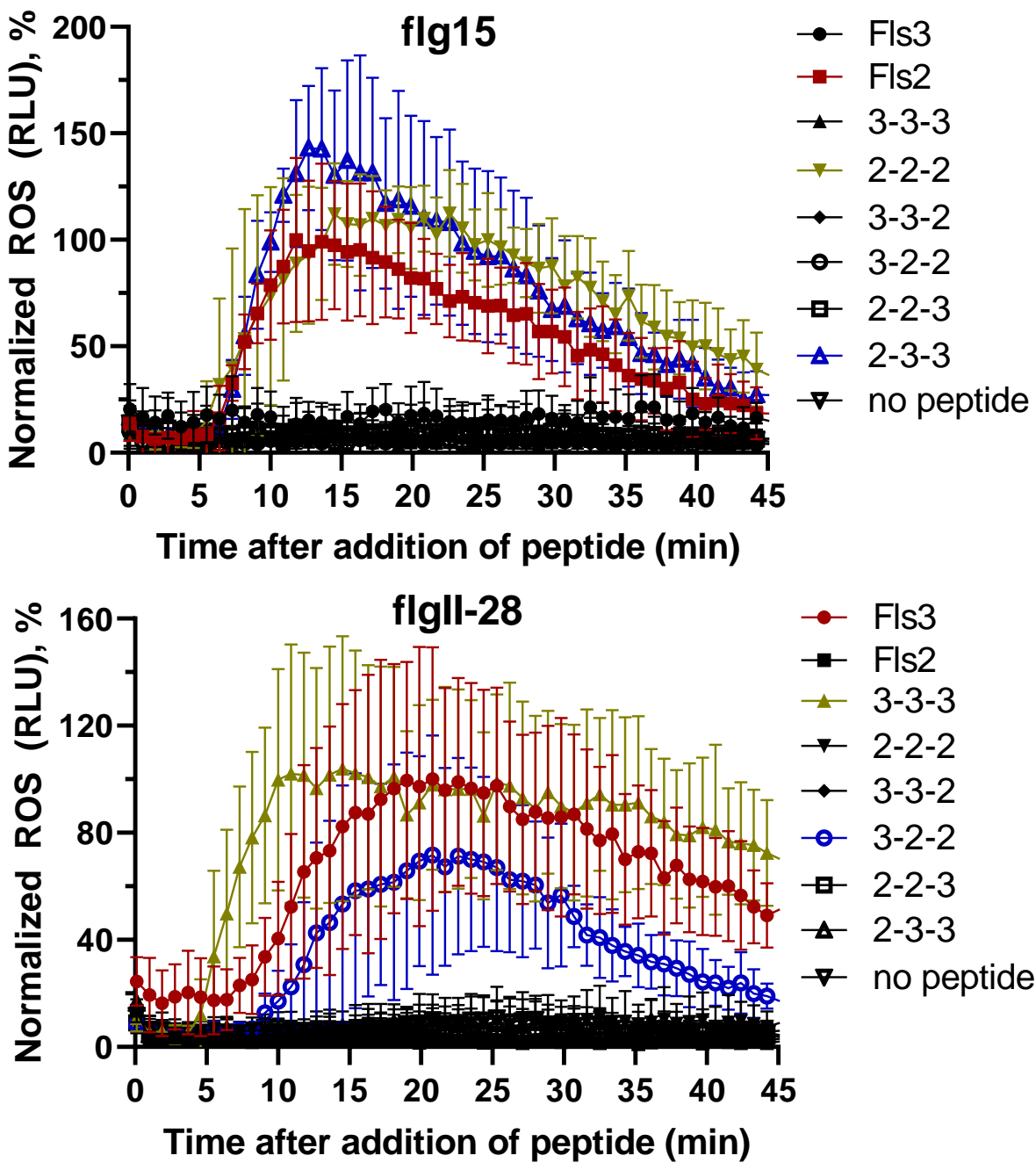

- Fls3

$\rightarrow$ Fls2

- 3-3-3

$\rightarrow 2-2-2$

$\rightarrow$ 3-3-2

- $3-2-2$

- 2-2-3

$\triangle$ 2-3-3

$\nabla$ no peptide

$$
45
$$


bioRxiv preprint doi: https://doi.org/10.1101/2020.02.04.934133; this version posted February 5, 2020. The copyright holder for this preprint A (which was not certified by peer review) is the author/funder, who has granted bioRxiv a license to display the preprint in perpetuity. It is made available under aCC-BY-ND 4.0 International license.

\section{Fls2.1}

Fls2.1

$\triangle \mathrm{Fls} 2.1$

Fls2.1

$\triangle \mathrm{F} 1 \mathrm{~s} 2.1$

Fls2.1/2.2

Fls2.1

$\triangle \mathrm{Fls} 2.1 / 2.2$

Fls2.2

$\triangle \mathrm{F} 1 \mathrm{~s} 2.1 / 2.2$

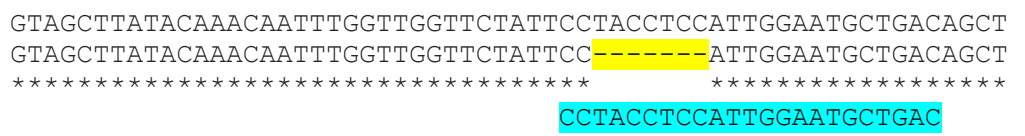

TTAGAAGCTTATAGAAACAATTTGGTTGGTTCTATTCCTACC-TCCATTGGAATGCTGAC TTAGAAGCTTATAGAAACAATTTGGTTGGTTCTATTCCTACCATCCATTGGAATGCTGAC

CCTACC TCCATTGGAATGCTGAC

Fls3

F1s3

$\triangle \mathrm{Fls} 3$
AAGACAGTTGTATATGCATTAGCCATATTTTCTATTACCTTTCTCATACCTTTGTCATCT AAGACAGTTGTATATGCATTAGCCATATTTTCTATTACCTTTCTCATA-------CATCT $* * * * * * * * * * * * * * * * * * * * * * * * * * * * * * * * * * * * * * * * * * * * * * * *$
CCTTTGTCATCT

GGGCAAAATCCAAGATTTGAAGTTGAAGTTGCTGCTTTGAAAGCTTTCAAGAGCTCAATC GGGCAAAATCCAAGATTTGAAGTTGAAGTTGCTGCTTTGAAAGCTTTCAAGAGCTCAATC

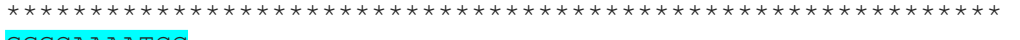
GGGCAAAATCC
ATGAGACTGATCAAGAAGCTCTACTAGCTTTTCGAAACCTTGT-TACGAGTGATTCTAGTC ATGAGACTGATCAAGAAGCTCTACTAGCTTTTCGAAACCTTGTCTACGAGTGATTCTAGTC

\section{Fls2.1/2.2/3}

Fls2.1
$\triangle F l s 2.1 / 2.2 / 3$
$F 1 s 2.2$
$\Delta F 1 s 2.1 / 2.2 / 3$
$F 1 s 3$
$\triangle F l s 2.1 / 2.2 / 3$

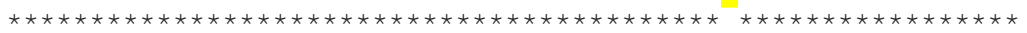

CCTTGT TACGAGTGATTCTAGTC

TTTGGTTGGTTTTATGCCTACCT-CCATTGGAATGCTGACAGCTTTGCATACCCTTGA
TTTGGTTGGTTTTATGCCTACCTTCCATTGGAATGCTGACAGCTTTGCATACCCTTGA
$* * * * * * * * * * * * * * * * * * * * * * * * * * * * * * * * * * * * * * * * * * * * * * *$
CCTACCT CCATTGGATGCTGAC

AGAAACAATTTGGTTGGTTCTATTCCTACCT-CCATTGGAATGCTGACAGCACTGCAAAC AGAAACAATTTGGTTGGTTCTATTCCTACCTTCCATTGGAATGCTGACAGCACTGCAAAC

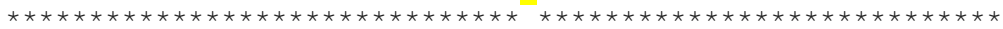
CCTACCT CCATTGGAATGCTGAC

GAAACCTTGT-TACGAGTGATTCTAGTCATTTTTTAGCCAATAATTGGACAAAAAACACTT GAAACCTTGTCTACGAGTGATTCTAGTCATTTTTTAGCCAATAATTGGACAAAAAACACTT

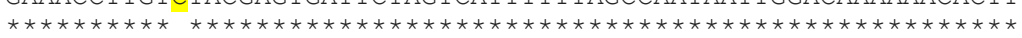
CCTTGT TACGAGTGATTCTAGTC

Figure S1. Development and characterization of CRISPR/Cas9-generated mutations in Fls2.1, Fls2.2 and Fls3. Related to Figure 1. (A) Alignment of CRISPR/Cas9-induced mutations (yellow) and gRNA target (blue) against each gene sequence. ((B), next page) (B) ROS showing loss of flg22 and flgII-28 perception in Rio Grande-prf3 tomato plants with mutations in Fls2.1 ( $\Delta$ Fls2.1), Fls3 ( $\Delta$ Fls3), Fls2.1 and Fls2.2 $(\Delta \mathrm{Fls} 2.1 / 2.2)$, or Fls2.1, Fls2.2, and Fls3 ( $\Delta$ Fls2.1/2.2/3). Shown are ROS produced in response to $100 \mathrm{nM}$ flgII-28 or flg22 peptide or water in the mutant plants (in the background of Rio Grande-prf3), Rio Grande-prf3, Yellow Pear, or $\Delta$ Fls2 in the background of M82 sp+ (Jacobs et al 2017). Results shown are the means of individual plants indicated as separate points, and horizontal lines are the means of the plants +/- s.d. $(\mathrm{n}=11 \mathrm{~T} 1$ plants for $\Delta \mathrm{Fls} 2.1 ; \mathrm{n}=4 \mathrm{~T} 2$ plants for $\Delta \mathrm{Fls} 3$ and $\Delta \mathrm{Fls} 2.1 / 2.2 ; \mathrm{n}=13 \mathrm{~T} 3$ plants for $\Delta \mathrm{Fls} 2.1 / 2.2 / 3 ; \mathrm{n}=2$ for Rio Grande, Yellow Pear, and $\Delta$ Fls2). Significance was determined via one-way ANOVA with Tukey's multiple comparisons test. 
B

$\Delta \mathrm{Fls} 2.1$

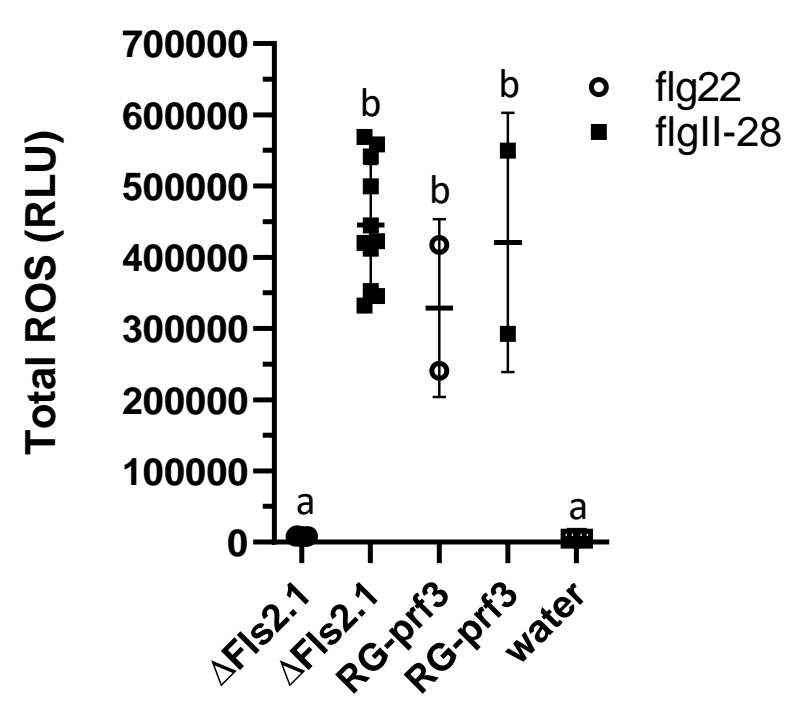

$\Delta$ Fls2.1/2.2

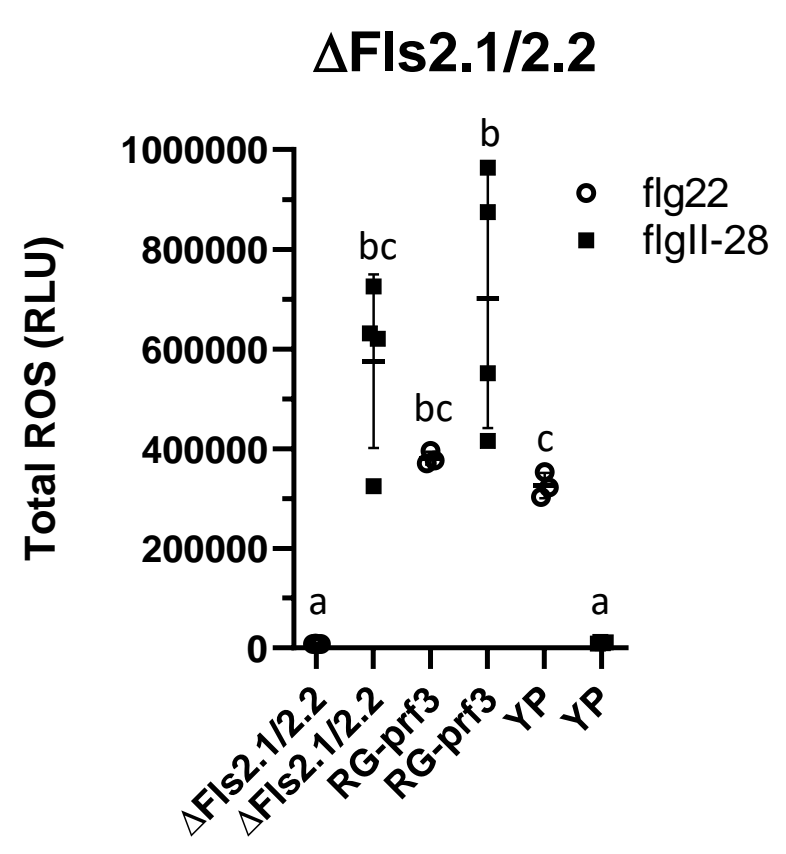

\section{$\Delta$ Fls3}

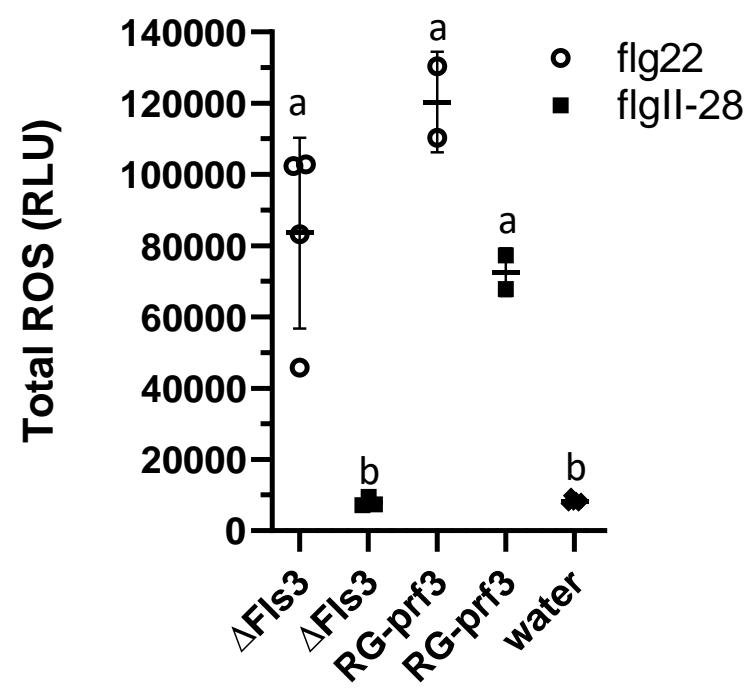

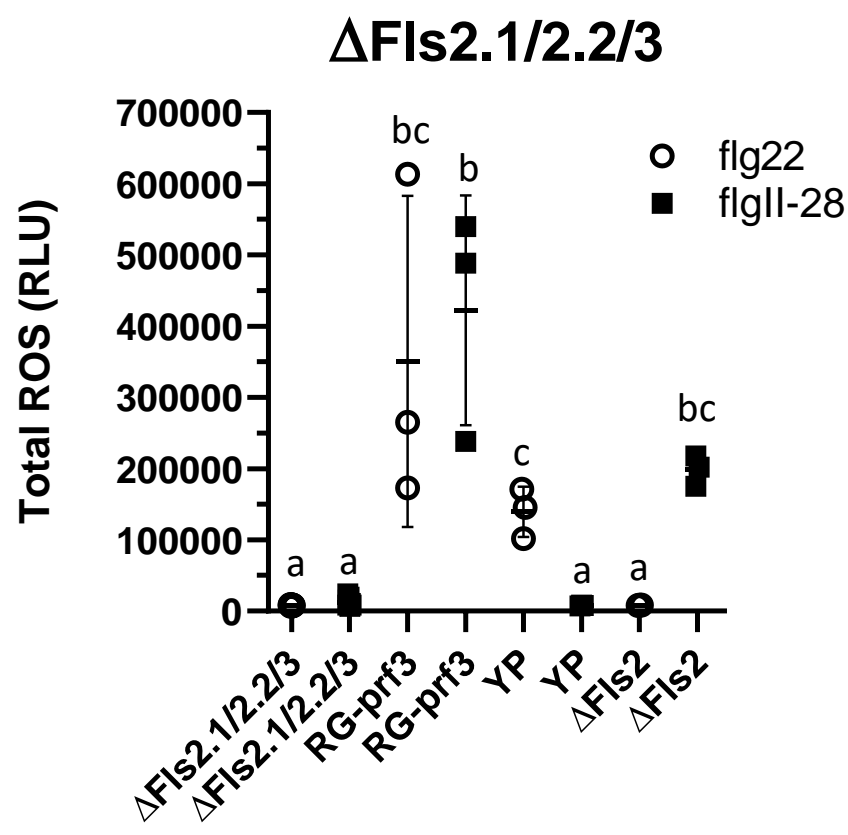

Figure S1. Development and characterization of CRISPR/Cas9-generated mutations in Fls2.1, Fls2.2 and Fls3. Related to Figure 1. ((A), previous page) Alignment of CRISPR/Cas9-induced mutations (yellow) and gRNA target (blue) against each gene sequence. (B) ROS showing loss of flg22 and flgII-28 perception in Rio Grande-prf3 tomato plants with mutations in $F l s 2.1$ $(\Delta$ Fls2.1), $F l s 3(\Delta$ Fls3), Fls2.1 and $F l s 2.2(\Delta$ Fls2.1/2.2), or $F l s 2.1, F l s 2.2$, and $F l s 3$ ( $\Delta$ Fls2.1/2.2/3). Shown are ROS produced in response to $100 \mathrm{nM}$ flgII-28 or flg22 peptide or water in the mutant plants (in the background of Rio Grande-prf3), Rio Grandeprf3, Yellow Pear, or $\Delta$ Fls2 in the background of M82 sp+ (Jacobs et al 2017). Results shown are the means of individual plants indicated as separate points, and horizontal lines are the means of the plants $+/$ - s.d. $(\mathrm{n}=11 \mathrm{~T} 1$ plants for $\Delta \mathrm{Fls} 2.1$; $\mathrm{n}=4 \mathrm{~T} 2$ plants for $\Delta \mathrm{Fls} 3$ and $\Delta \mathrm{Fls} 2.1 / 2.2 ; \mathrm{n}=13 \mathrm{~T} 3$ plants for $\Delta \mathrm{Fls} 2.1 / 2.2 / 3 ; \mathrm{n}=2$ for Rio Grande, Yellow Pear, and $\Delta \mathrm{Fls} 2)$. Significance was determined via one-way ANOVA with Tukey's multiple comparisons test. 


\section{A}

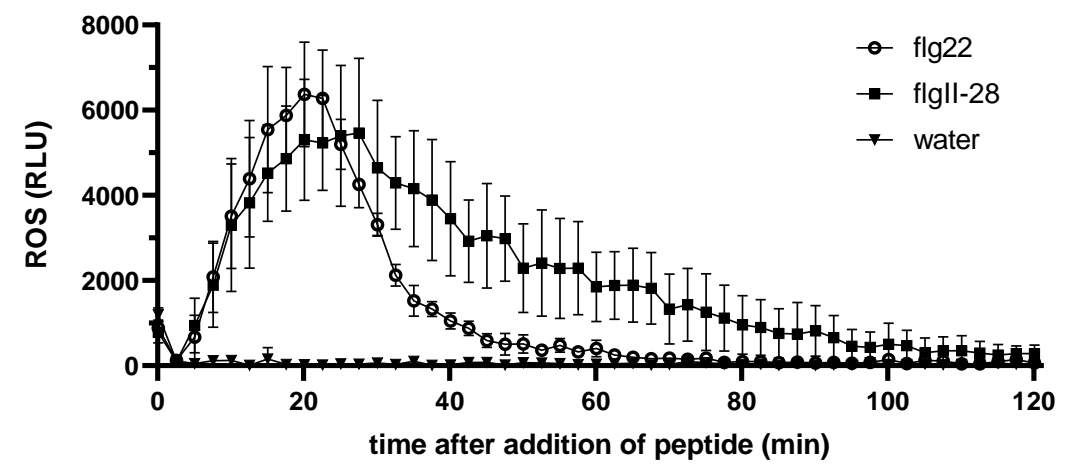

C Potato (Dakota Crisp)

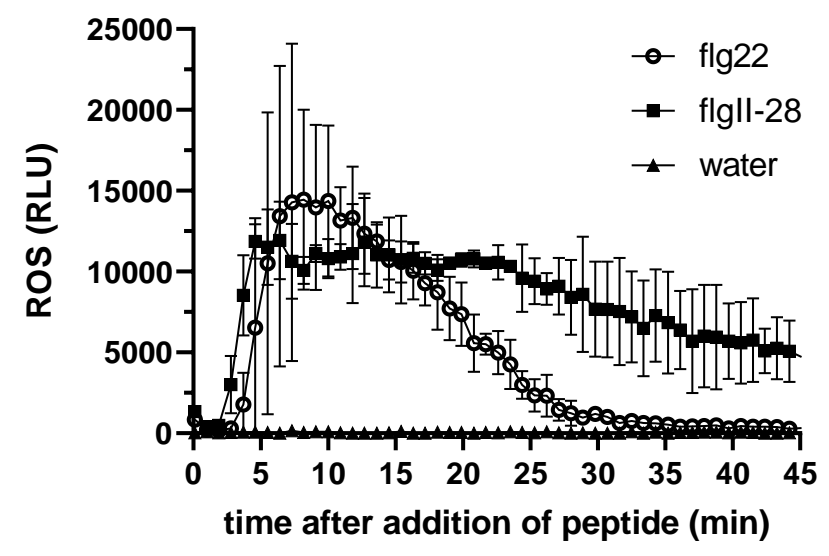

B
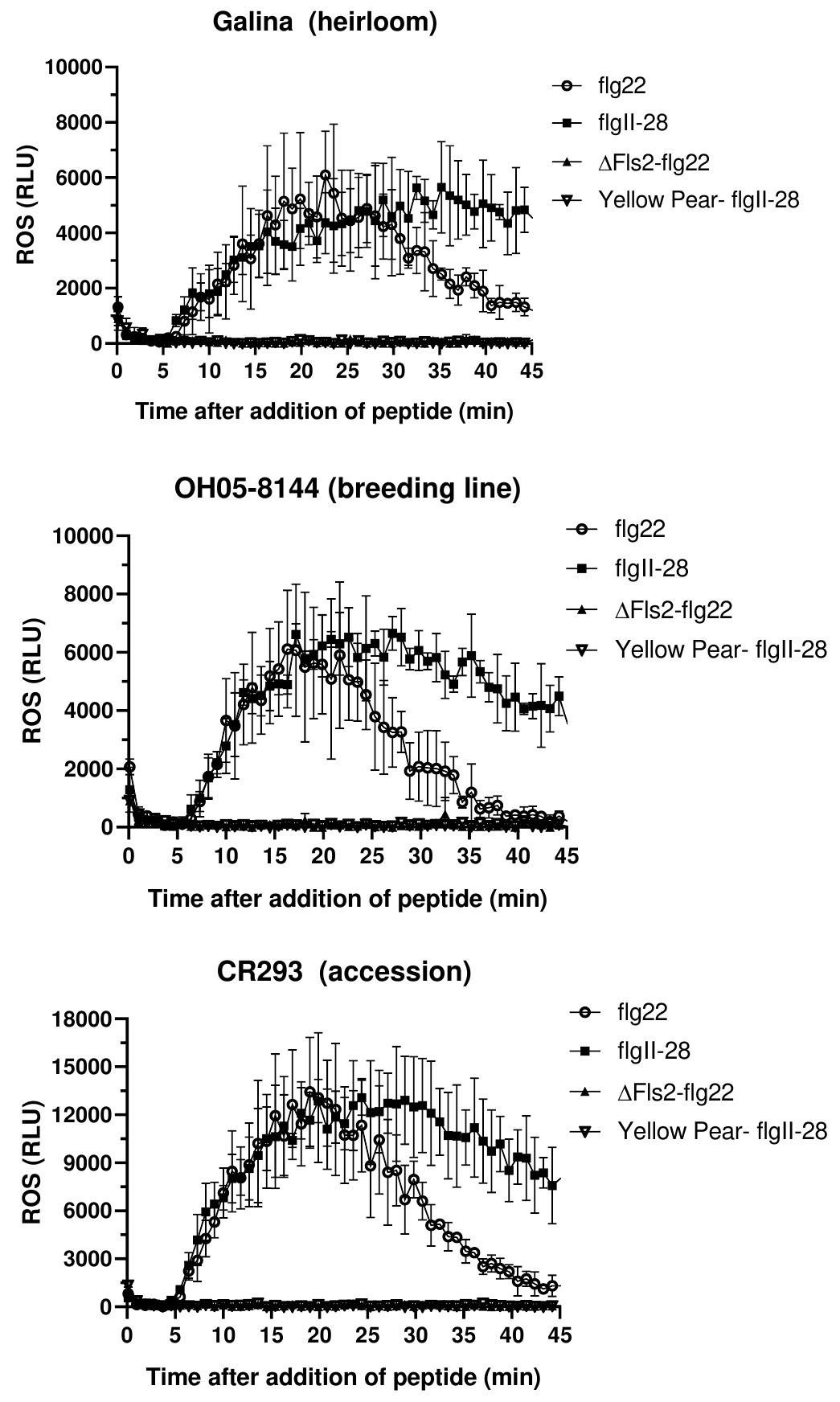

Figure S2. Additional tomato and potato accessions with sustained flgII-28 ROS response. Related to Figure 2. Oxidative burst (ROS) produced in response to $100 \mathrm{nM}$ flgII-28 or flg22 peptide or water in tomato and potato. (A) The tomato Rio Grande-prf3 flgII-28 response is sustained for 100 minutes, compared to 60 min for flg22. (B) Tomato heirloom Galina, breeding line OH05-8144, and accession CR293, or (C) Potato var. Dakota Crisp, all have sustained ROS responses to flgII-28. ROS was measured in relative light units (RLU). Results shown are means $+/$ - s.d. ( $\mathrm{n}=3$ for tomato, $\mathrm{n}=2$ for potato) and are representative of two (tomato) or three (potato) independent experiments. 


\section{Eggplant var. Shikou}

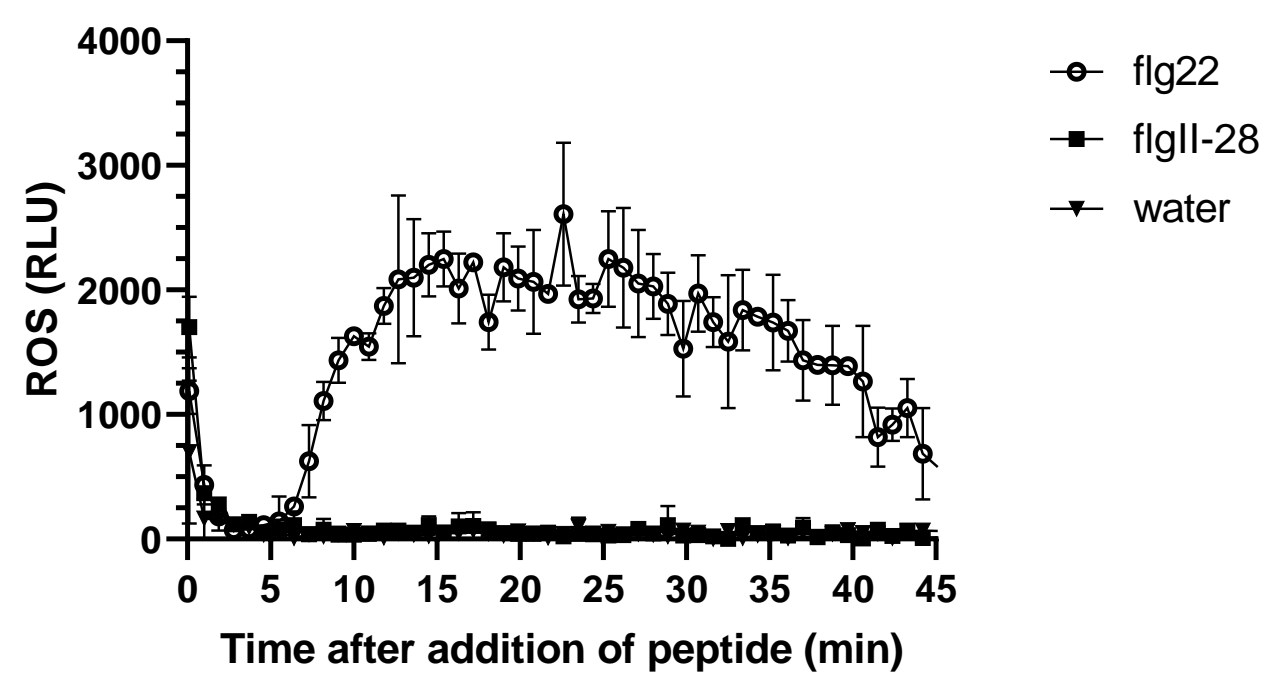

Figure S3. Eggplant var. Shikou does not respond to flgII-28. Related to Figure 2. Oxidative burst (ROS) produced in response to $100 \mathrm{nM}$ flgII-28 or flg22 peptide or water, measured in relative light units (RLU). Results shown are means $+/$ - s.d. ( $n=3$ plants). The four oldest leaves from each of three 6-week-old plants were tested, and shown are the results from leaf 4 . Similar observations were made for each of the four leaves. 
bioRxiv preprint doi: https://doi.org/10.1101/2020.02.04.934133; this version posted February 5, 2020. The copyright holder for this preprint (which was not certified by peer review) is the author/funder, who has granted bioRxiv a license to display the preprint in perpetuity. It is made

A
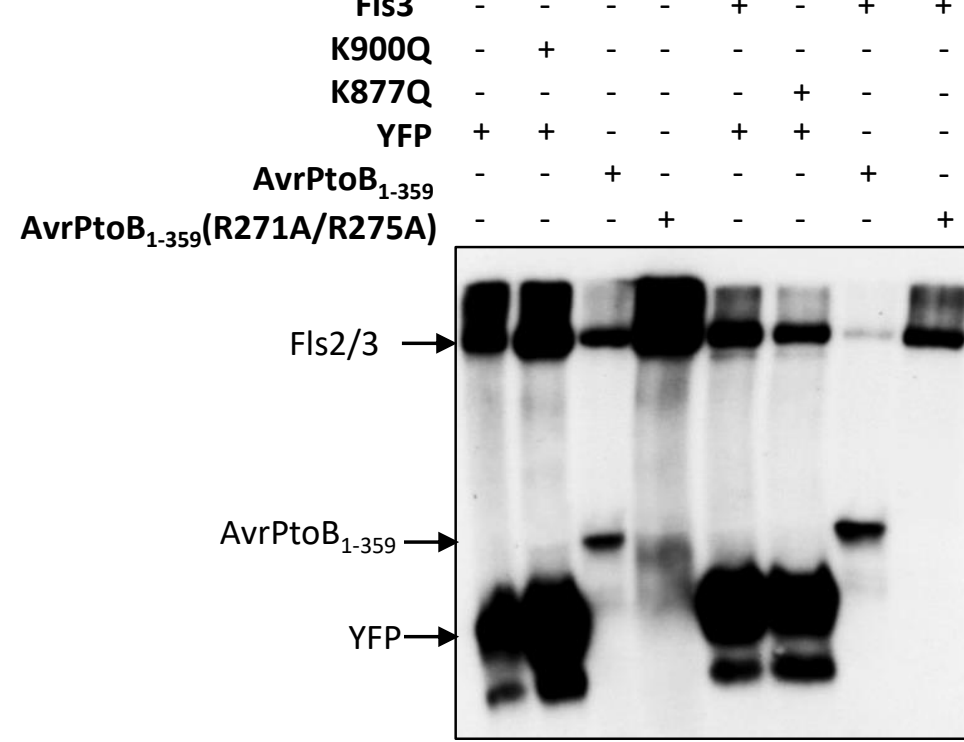

WB: $\alpha-m y c$

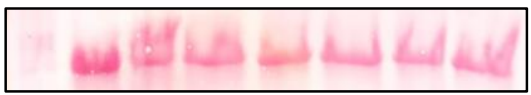

Ponceau

B

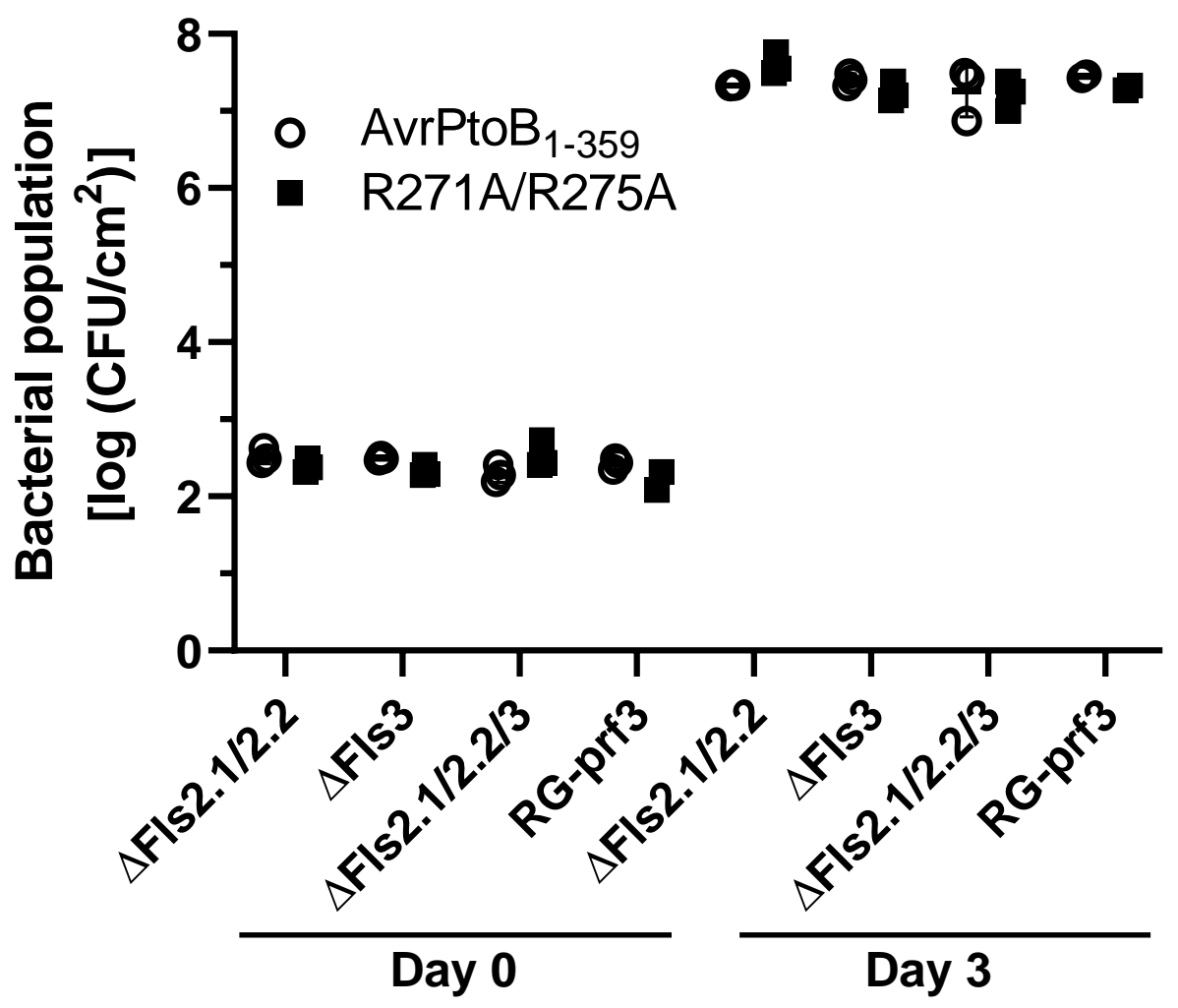

C

\begin{tabular}{|c|c|c|c|c|}
\hline & \multicolumn{2}{|c|}{ DC3000::AvrPtoB $_{\text {1-359 }}$} & \multicolumn{2}{c|}{$\begin{array}{c}\text { DC3000::AvrPtoB }_{\text {1-359 }} \\
\text { (R271A/R275A) }\end{array}$} \\
\hline CRISPR/Cas9 & Average & SD & Average & SD \\
\hline$\Delta$ Fls2.1/2.2 & 7.33 & \pm 0.01 & 7.60 & \pm 0.14 \\
\hline$\Delta$ Fls3 & 7.40 & \pm 0.08 & 7.24 & \pm 0.12 \\
\hline$\Delta$ FIs2.1/2.2/3 & 7.26 & \pm 0.34 & 7.21 & \pm 0.19 \\
\hline RG-prf3 & 7.45 & \pm 0.03 & 7.30 & \pm 0.04 \\
\hline
\end{tabular}

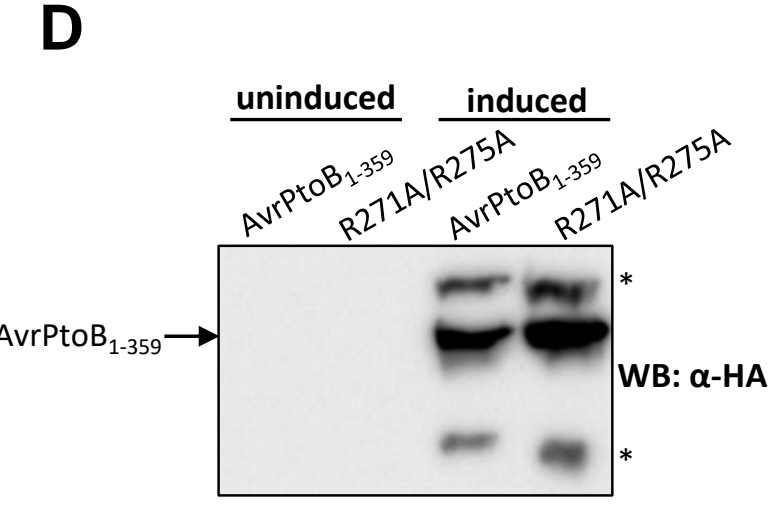

Ponceau

Figure S4. Supplementary information related to Figure 4. (A) Immunoblots showing protein expression of constructs in Figure 4A. 10 $\mu \mathrm{g}$ of total protein extracted from $N$. benthamiana leaves coagroinfiltrated and overexpressed with Fls2, Fls3, Fls2(K900Q), Fls3(K887Q), or YFP and AvrPtoB $_{1-}$ 359 or $\mathrm{AvrPtoB}_{1-359}(\mathrm{R} 271 \mathrm{~A} / \mathrm{R} 275 \mathrm{~A})$ was run on SDSPAGE and immunoblotted using an anti-cMyc antibody. Ponceau stain of the PVDF membrane was performed to compare loading. Constructs were overexpressed under a $35 \mathrm{~S}$ promoter. (B) Bacterial populations in CRISPR/Cas9 mutant tomato plants Fls2.1 and Fls2.2 ( $\Delta \mathrm{Fls} 2.1 / 2.2), F l s 3(\Delta \mathrm{Fls} 3)$, or Fls2.1, Fls2.2, and $F l_{s} 3(\Delta \mathrm{Fls} 2.1 / 2.2 / 3)$ or wild type Rio Grande-prf3 (RG-prf3). Bacteria were measured 0 and 3 days after vacuum-infiltrating plants with bacterial suspensions $\left(1 \times 10^{4} \mathrm{CFU} / \mathrm{mL}\right)$ using a DC3000 strain expressing AvrPtoB ${ }_{1-359}$ (labeled AvrPtoB ${ }_{1-359}$ ) or its variant $\mathrm{AvrPtoB}_{1-}$ ${ }_{359}(\mathrm{R} 271 \mathrm{~A} / \mathrm{R} 275 \mathrm{~A})$ (labeled R271A/R275A). Shown are the means of three individual plants indicated as separate points, and horizontal lines are the means of the three plants +/- s.d.. There were no statistically significant differences using a pairwise t-test in bacterial growth between the two strains for each plant $(P>0.05)$. (C) Table showing the averages and standard deviations (SD) of the data in (B). (D) Immunoblot showing expression of $\mathrm{AvrPtoB}_{1-359}$ and $\mathrm{AvrPtoB}_{1-359}(\mathrm{R} 271 \mathrm{~A} / \mathrm{R} 275 \mathrm{~A})$ expressed from a plasmid in DC3000 before and after induction of the hrp inducible promoter in minimal media. The Cterminal HA tag was detected using an anti-HA antibody, and Ponceau staining was performed to compare loading. *Protein of unexpected size specifically detected in $\mathrm{AvrPtoB}_{1-359^{-}}$expressing bacteria, as previously reported in Cheng et al (2011). 
bioRxiv preprint doi: https://doi.org/10.1101/2020.02.04 934133; this version posted February 5, 2020. The copyright holder for this preprint (which was not certified by peer review) is the author/funder, who has granted bioRxiv a license to display the preprint in perpetuity. It is made available under aCC-BY-ND 4.0 International license.

\begin{tabular}{|c|c|c|}
\hline Consensus & $\begin{array}{l}\text { IL I GSGSFG } \\
\text { GxGxxG }\end{array}$ & G-TLIK D ĠTXIVAV \\
\hline Identity & & \\
\hline $\begin{array}{l}\text { 1. Solyc12g008400.2.1. } \\
\text { 2. Solyc11g020260.2.1 } \\
\text { 3. Solyc10g085120.2.1 } \\
\text { 4. Solyc08g075610.2.1. } \\
\text { 5. Solyc08g075600.2.1 } \\
\text { 6. Solyc08g075590.2.1. } \\
\text { 7. Solyc07g018190.3.1. } \\
\text { 8. Solyc07g018180.2.1. } \\
\text { 9. Solyc07g006770.2.1. } \\
\text { 10. Solyc07g005920.2.1 } \\
\text { 11. Solyc06g076910.2.1 } \\
\text { 12. Solyc06g048740.2.1 } \\
\text { 13. Solyc06g006040.2.1 } \\
\text { 14. Solyc06g006020.2.1 } \\
\text { 15. Solyc06g006010.2.1 } \\
\text { 16. Solyc05g007230.3.1 } \\
\text { 17. Solyc04g015980.3.1 } \\
\text { 18. Solyc04g014900.3.1 } \\
\text { 19. Solyc04g014650.3.1 } \\
\text { 20. Solyc04g012190.2.1 } \\
\text { 21. Solyc04g012110.2.1 } \\
\text { 22. Solyc04g012100.2.1 } \\
\text { 23. Solyc04g012090.2.1 } \\
\text { 24. Solyc04g009640.3.1 } \\
\text { Fls3 25. Solyc04g009040.3.1 } \\
\text { 26. Solyc03g098400.2.1 } \\
\text { 27. Solyc03g096190.2.1 } \\
\text { 28. Solyc03g019980.2.1 } \\
\text { 29. Solyc03g006100.3.1 } \\
\text { 30. Solyc03g006080.3.1 } \\
\text { 31. Solyc03g006030.3.1 } \\
\text { 32. Solyc02g072480.3.1 } \\
\text { 33. Solyc02g072470.3.1 } \\
\text { 34. Solyc02g072440.3.1 } \\
\text { 35. Solyc02g072400 } \\
\text { Is2.2 36. Solyc02g070910.3.1 } \\
\text { Is2.1 37. Solyc02g070890.3.1 } \\
\text { 38. Solyc02g068830.2.1 } \\
\text { 39. Solyc02g068820.2.1 } \\
\text { 40. Solyc02g031790.2.1 }\end{array}$ & 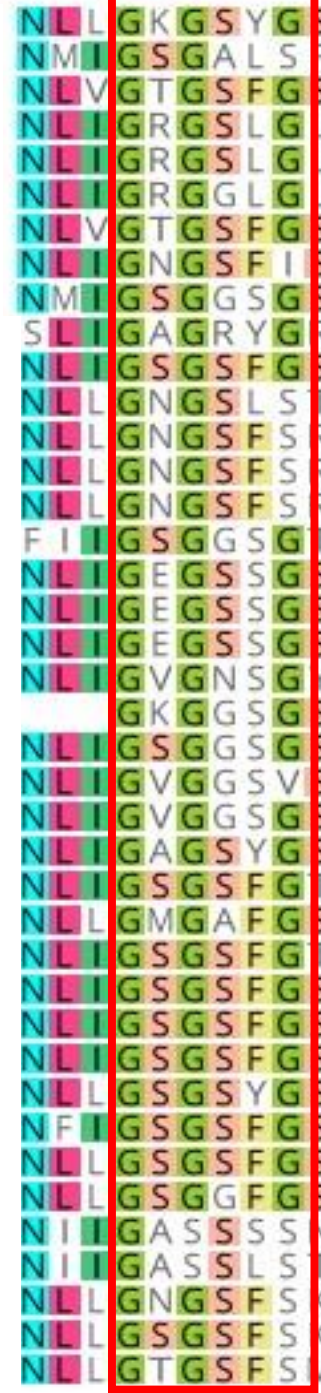 & 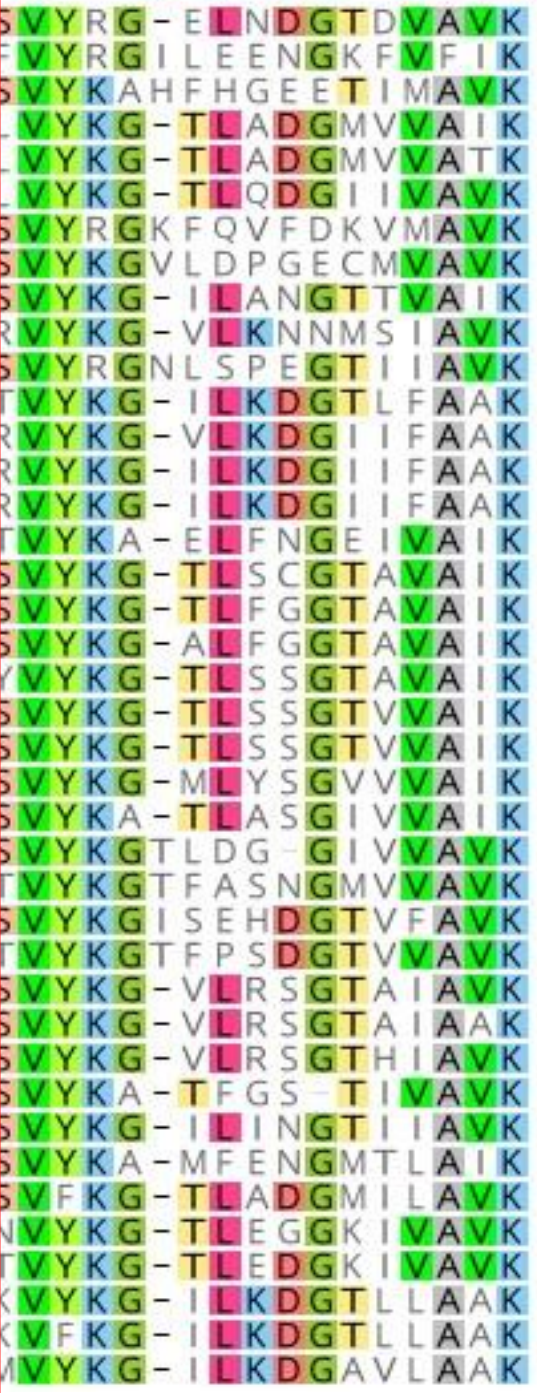 \\
\hline
\end{tabular}

B

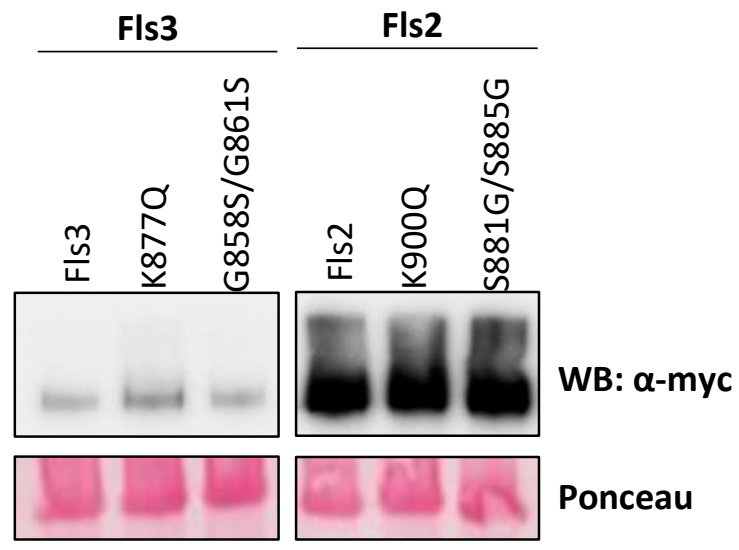

Figure S5. Supplementary information related to Figure 6. (A) Alignment of subdomain I of the RLKs present in class IX-a. The GxGxxG motif and the lysine (K) involved in ATP binding are annotated in yellow. The GxGxxG motif is also boxed in red and the lysine is indicated with a red arrow. Identity is shown with green indicating conservation, olive indicating high identity, and pink indicating low identity. Of the 42 RLKs in the class, two genes (Solyc10g085110 and Solyc03g118330) do not encode a subdomain I region or have the active lysine residue and are not included in the alignment. The alignment was assembled using the Geneious R11 software. (B) Immunoblot showing expression of proteins related to Figure $6.5 \mu \mathrm{g}$ of total protein was extracted from N. benthamiana leaves agroinfiltrated with each of the Fls3 and Fls2 constructs or their variants and run on SDS-PAGE. Proteins were detected using an anti-cMyc antibody and Ponceau staining of the PVDF membrane was performed to compare loading. Constructs were overexpressed under the control of the CaMV 35S promoter. All samples shown are from the same experiment. 
A Total ROS at Maximum Amplitude

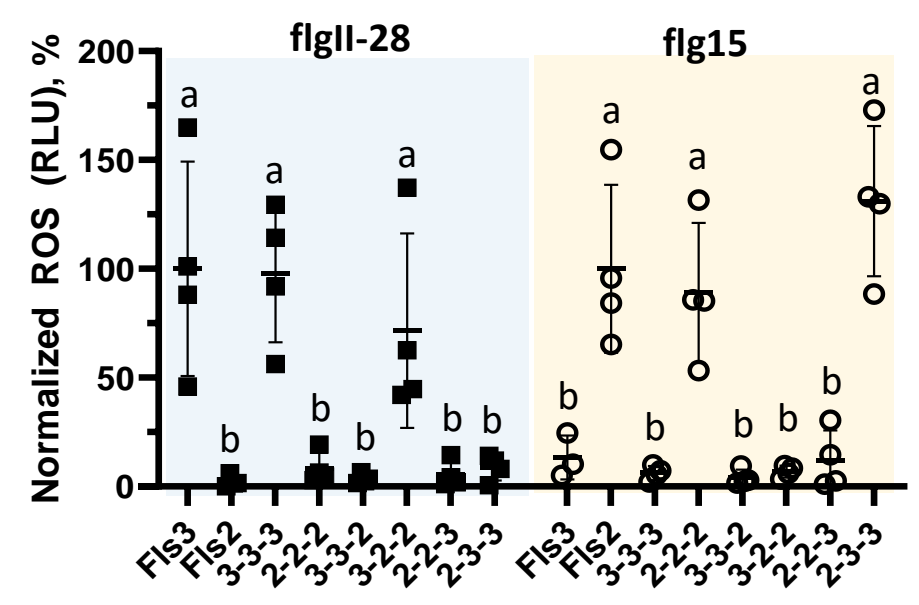

chimeric construct

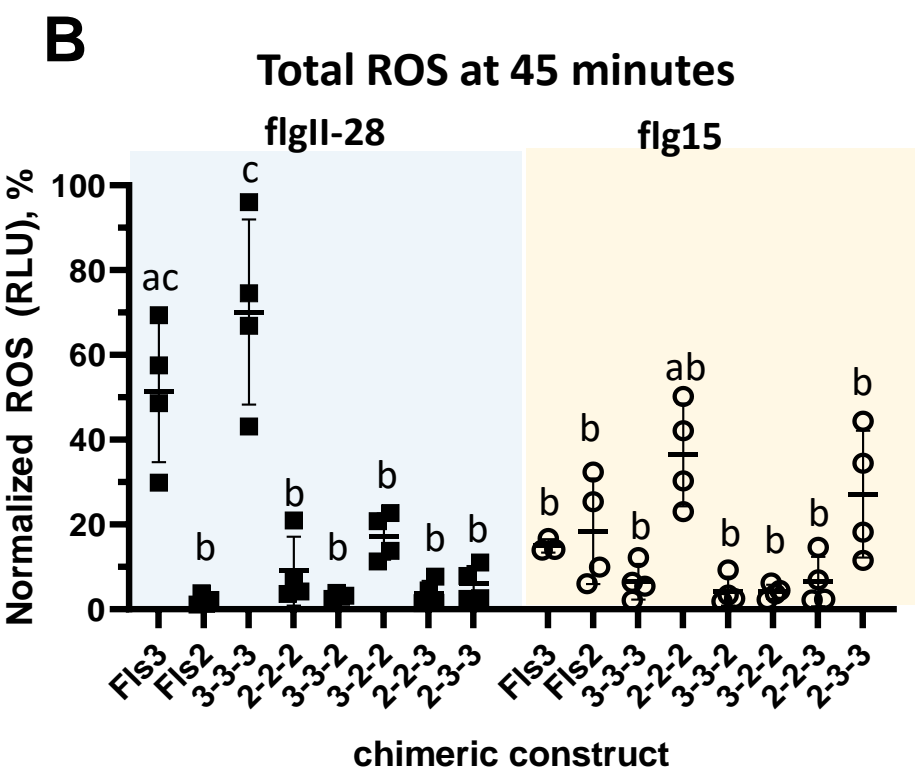

$3-3-2 \quad 3-2-2 \quad 2-2-3 \quad 2-3-3$

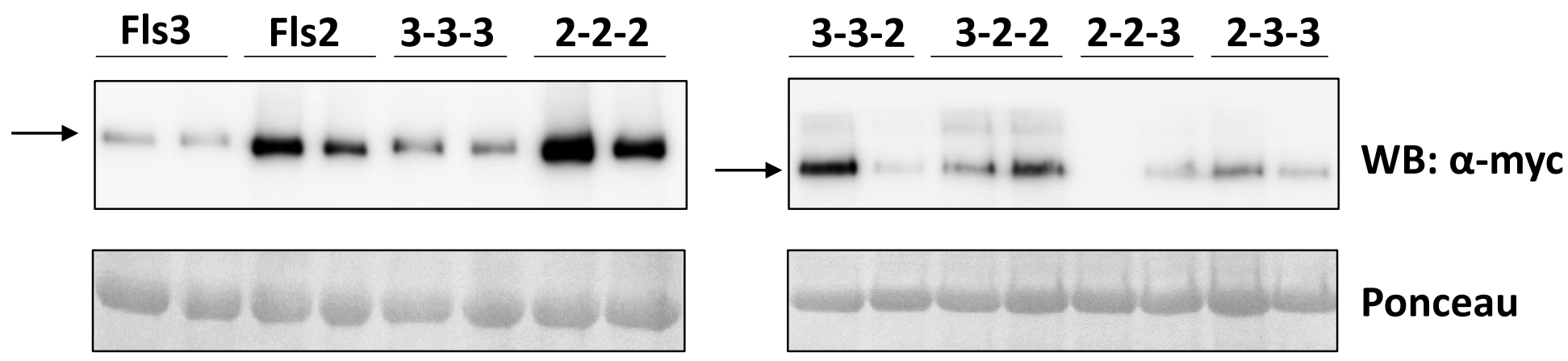

Figure S6. Only the chimeric constructs with a TM and KD originating from the same receptor respond to flgII-28 or flg15 peptide. Related to Figure 7. (A) Normalized total ROS response at the maximum amplitude for Fls3-flgII-28 or Fls2-flg15, $20 \mathrm{~min}$ (Fls3) or 12 min (Fls2) after peptide treatment (10nM flgII-28 or flg15). (B) Normalized total ROS response at 45 minutes after peptide treatment (10nM flgII-28 or flgII-15). Values in (A) and (B) were normalized to the maximum ROS (RLU) of the positive controls (Fls3 or Fls2) on each ROS plate. Constructs were transiently expressed in $N$. benthamiana under control of the CaMV $35 \mathrm{~S}$ promoter. Data shown are the means of four individual plants indicated as separate points, and horizontal lines are the means of the four plants $+/$ - s.d. ( $n=4$ plants). Statistical significance was determined by Two-way ANOVA followed by Tukey's multiple comparisons post-test. Data are representative of three independent experiments. Black squares signify samples treated with flgII-28, and open circles were samples treated with flg15. (C) Immunoblot showing expression of the chimeric constructs transiently expressed in N. benthamiana. Two samples from two individual plants (technical replicates) of the same experimental replicate (biological replicate) are shown for each infiltrated construct. $5 \mu \mathrm{g}$ of total protein was run on SDS-PAGE and proteins were detected using an anti-cMyc antibody. 
bioRxiv preprint doi: https://doi.org/10.1101/2020.02.04.934133; this version posted February 5, 2020. The copyright holder for this preprint (which was not certified by peer review) is the author/funder, who has granted bioRxiv a license to display the preprint in perpetuity. It is made available under aCC-BY-ND 4.0 International license.

\section{Reference}

Jacobs, T.B., Zhang, N., Patel, D., and Martin, G.B. (2017). Generation of a collection of mutant tomato lines using pooled CRISPR libraries. Plant Physiol 174:2023-2037. 


\begin{tabular}{|c|c|c|c|c|c|c|c|c|}
\hline CRISPR line & Solyc \# & Gene & Mutation & Mutation sequence & gRNA that induced the mutation & Cas9 & Morphology & $\begin{array}{l}\text { Immune } \\
\text { response }\end{array}$ \\
\hline$\Delta \mathrm{Fls} 2.1$ & Solyc02g070890 & Fls 2.1 & -7 & ССТТТСТСАТА(-7bp)САТСТ & GGATTTTGCCCAGATGACAA & - & normal & $\begin{array}{c}\text { flg22 } \\
\text { insensitive }\end{array}$ \\
\hline \multirow{2}{*}{$\Delta$ Fls2.1/2.2 } & Solyc02g070890 & Fls2.1 & -7 & GGTTCTATTCC(-7bp)ATTGG & \multirow{2}{*}{ GTCAGCATTCCAATGGAGGT } & \multirow[b]{2}{*}{+} & \multirow{2}{*}{$\begin{array}{l}\text { few fruit, many } \\
\text { parthenocarpic }\end{array}$} & \multirow{2}{*}{$\begin{array}{c}\text { flg22 } \\
\text { insensitive }\end{array}$} \\
\hline & Solyc02g070910 & $F l s 2.2$ & +1 & TCCTACC+ATCCATTGG & & & & \\
\hline$\Delta \mathrm{Fls} 3$ & Solyc04g009640 & Fls3 & +1 & AAACCTTGT+CTACGAG & GACTAGAATCACTCGTAACA & - & normal & $\begin{array}{c}\text { flgII-28 } \\
\text { insensitive }\end{array}$ \\
\hline \multirow{3}{*}{$\Delta \mathrm{Fls} 2.1 / 2.2 / 3$} & Solyc04g009640 & Fls3 & +1 & GAAACCTTGT+CTACGAG & GACTAGAATCACTCGTAACA & & \multirow{3}{*}{ normal } & \multirow{3}{*}{$\begin{array}{l}\text { flg22 and } \\
\text { flgII-28 } \\
\text { insensitive }\end{array}$} \\
\hline & Solyc02g070890 & Fls 2.1 & +1 & TGCCTACCT+TCCATTGGAATG & \multirow{2}{*}{ GTCAGCATTCCAATGGAGGT } & - & & \\
\hline & Solyc02g070910 & Fls 2.2 & +1 & CCTACCT+TCCATTGGAATG & & & & \\
\hline
\end{tabular}




\begin{tabular}{|c|c|c|c|c|c|c|c|c|}
\hline GeneID & Human readable description & Mock 6h & flg22 $6 h$ & ratio & adjust $\mathbf{p}$ & flgII $286 h$ & ratio & adjust $p$ \\
\hline Solyc03g114230.1.1 & $\begin{array}{l}\text { BHLH transcription factor (AHRD V1 } * * * * \\
\text { A9JX09_ORYSJ); contains Interpro domain(s) } \\
\text { IPR011598 Helix-loop-helix DNA-binding }\end{array}$ & 0.02 & 0.26 & 11.56 & 0.7641042 & 22.67 & 1007.41 & $2.52 \mathrm{E}-50$ \\
\hline Solyc09g098080.2.1 & $\begin{array}{l}\text { UDP-glucosyltransferase (AHRD V1 } * * * * \\
\text { A7M6U9_IPONI); contains Interpro domain(s) } \\
\text { IPR002213 UDP-glucuronosyl/UDP-glucosyltransferase }\end{array}$ & 0.01 & 0.25 & 20 & 0.2636374 & 4.99 & 398.93 & $3.87 \mathrm{E}-19$ \\
\hline Solyc12g088850.1.1 & $\begin{array}{l}\text { EF hand family protein (AHRD V1 } * *_{--} \\
\text {B4G1S0_MAIZE); contains Interpro domain(s) } \\
\text { IPR011992 EF-Hand type }\end{array}$ & 0 & 0 & 1 & NA & 3.72 & 372 & $1.04 \mathrm{E}-06$ \\
\hline Solyc09g074500.1.1 & Unknown Protein (AHRD V1) & 0 & 0.57 & 57 & 0.7099617 & 3.06 & 306 & $6.23 \mathrm{E}-05$ \\
\hline Solyc04g079260.2.1 & $\begin{array}{l}\text { Patatin-like protein } 1 \text { (AHRD V1 } * * * \text { - } \\
\text { Q9FZ09_TOBAC); contains Interpro domain(s) } \\
\text { IPR002641 Patatin }\end{array}$ & 0.06 & 0.06 & 1 & 1 & 4.02 & 73.03 & $1.60 \mathrm{E}-11$ \\
\hline Solyc05g052650.2.1 & $\begin{array}{l}\text { N-hydroxycinnamoyl/benzoyltransferase } 6 \text { (AHRD V1 } \\
\text { ***-Q00M84_SOYBN); contains Interpro domain(s) } \\
\text { IPR003480 Transferase }\end{array}$ & 0.06 & 0.27 & 4.91 & 0.8573557 & 3.85 & 70.06 & 0.0015624 \\
\hline Solyc08g036620.2.1 & $\begin{array}{l}\text { Protein TIFY 5A (AHRD V1 } * * * \text { - TIF5A_ARATH); } \\
\text { contains Interpro domain(s) IPR010399 Tify }\end{array}$ & 0.08 & 0.61 & 7.69 & 0.8230538 & 3.12 & 39 & $1.09 \mathrm{E}-05$ \\
\hline Solyc05g053330.2.1 & $\begin{array}{l}\text { MYB transcription factor (AHRD V1 } * * *- \\
\text { Q39550_CRAPL); contains Interpro domain(s) } \\
\text { IPR015495 Myb transcription factor }\end{array}$ & 0.17 & 0.1 & 0.54 & 1 & 6.36 & 36.32 & $2.26 \mathrm{E}-05$ \\
\hline Solyc10g011930.1.1 & $\begin{array}{l}\text { Phenylalanine ammonia-lyase (AHRD V1 } * * *- \\
\text { B5LAW0_CAPAN); contains Interpro domain(s) } \\
\text { IPR001106 Phenylalanine/histidine ammonia-lyase }\end{array}$ & 0.14 & 0.93 & 6.49 & 0.6823666 & 4.3 & 30.18 & $3.50 \mathrm{E}-07$ \\
\hline Solyc01g095080.2.1 & $\begin{array}{l}\text { 1-aminocyclopropane-1-carboxylate synthase (AHRD V1 } \\
* * * * \text { O65209_NICGU); contains Interpro domain(s) } \\
\text { IPR004839 Aminotransferase, class I and II IPR004838 } \\
\text { Aminotransferases, class-I, pyridoxal-phosphate-binding } \\
\text { site }\end{array}$ & 0.11 & 0.06 & 0.57 & 1 & 3 & 28.6 & $1.16 \mathrm{E}-16$ \\
\hline Solyc10g008260.1.1 & $\begin{array}{l}\text { Helix-loop-helix DNA-binding (AHRD V1 } * * * \text { - } \\
\text { Q2HTT3_MEDTR); contains Interpro domain(s) } \\
\text { IPR001092 Basic helix-loop-helix dimerisation region } \\
\text { bHLH }\end{array}$ & 0.23 & 0.89 & 3.77 & 0.5468346 & 4.75 & 20.23 & $1.91 \mathrm{E}-11$ \\
\hline Solyc07g064650.1.1 & $\begin{array}{l}\text { Arabidopsis thaliana genomic DNA chromosome } 5 \text { P1 } \\
\text { clone MOK16 (AHRD V1 *--- Q9LYW2_ARATH); } \\
\text { contains Interpro domain(s) IPR007608 Protein of } \\
\text { unknown function DUF584 }\end{array}$ & 0.85 & 3.04 & 3.59 & 0.7342515 & 14.97 & 17.66 & $6.66 \mathrm{E}-21$ \\
\hline
\end{tabular}




\begin{tabular}{|c|c|c|c|c|c|c|c|c|}
\hline Solyc08g067630.2.1 & Unknown Protein (AHRD V1) & 1.52 & 2.01 & 1.32 & 1 & 22.74 & 14.94 & $8.48 \mathrm{E}-15$ \\
\hline Solyc08g075550.2.1 & $\begin{array}{l}\text { Alternative oxidase (AHRD V1 } * * * * \text { Q84V46_SOLLC); } \\
\text { contains Interpro domain(s) IPR002680 Alternative } \\
\text { oxidase }\end{array}$ & 1.31 & 1.46 & 1.11 & 1 & 18 & 13.69 & 8.79E-08 \\
\hline Solyc09g082270.2.1 & $\begin{array}{l}\text { Non-specific lipid-transfer protein (AHRD V1 } * *_{-} \\
\text {B9SFJ1_RICCO); contains Interpro domain(s) } \\
\text { IPR013770 Plant lipid transfer protein and hydrophobic } \\
\text { protein, helical }\end{array}$ & 2.73 & 1.56 & 0.57 & 1 & 36.48 & 13.36 & 0.008666 \\
\hline Solyc12g010970.1.1 & $\begin{array}{l}\text { Alcohol dehydrogenase-like protein (AHRD V1 } * *_{-} \\
\text {Q2KNL2_OCIBA); contains Interpro domain(s) } \\
\text { IPR002085 Alcohol dehydrogenase superfamily, zinc- } \\
\text { containing }\end{array}$ & 0.38 & 0.35 & 0.92 & 1 & 4.31 & 11.33 & 0.0385502 \\
\hline Solyc03g117860.2.1 & $\begin{array}{l}\text { IBR finger domain protein (AHRD V1 } * *-- \\
\text { Q4WL53_ASPFU); contains Interpro domain(s) } \\
\text { IPR002867 Zinc finger, C6HC-type }\end{array}$ & 4.98 & 3.62 & 0.73 & 1 & 53.18 & 10.68 & $8.97 \mathrm{E}-33$ \\
\hline Solyc05g014590.2.1 & $\begin{array}{l}\text { Transcription Factor (AHRD V1 } * * * * \\
\text { Q84LF9_ORYSA); contains Interpro domain(s) } \\
\text { IPR011598 Helix-loop-helix DNA-binding }\end{array}$ & 1.99 & 2.94 & 1.47 & 1 & 20.84 & 10.46 & $6.47 \mathrm{E}-16$ \\
\hline Solyc03g098720.2.1 & $\begin{array}{l}\text { Kunitz trypsin inhibitor (AHRD V1 } * * *_{-} \\
\text {Q4W188_POPTN); contains Interpro domain(s) } \\
\text { IPR011065 Kunitz inhibitor ST1-like }\end{array}$ & 0.47 & 1.16 & 2.48 & 0.7503725 & 4.82 & 10.31 & 0.0031629 \\
\hline Solyc10g076510.1.1 & $\begin{array}{l}\text { Pyruvate decarboxylase (AHRD V1 } * * * * \\
\text { B0ZS79_PRUAR); contains Interpro domain(s) } \\
\text { IPR012110 Pyruvate decarboxylase/indolepyruvate } \\
\text { decarboxylase }\end{array}$ & 2.45 & 2.32 & 0.94 & 1 & 25.16 & 10.26 & $2.31 \mathrm{E}-06$ \\
\hline Solyc03g119370.1.1 & $\begin{array}{l}\text { MYB transcription factor (AHRD V1 } *_{-} *_{-} \\
\text {Q9C9G7_ARATH); contains Interpro domain(s) } \\
\text { IPR015495 Myb transcription factor }\end{array}$ & 1.13 & 1.27 & 1.12 & 1 & 10.97 & 9.68 & $2.18 \mathrm{E}-08$ \\
\hline Solyc10g005320.2.1 & $\begin{array}{l}\text { Tryptophan synthase beta chain } 1 \text { (AHRD V1 } * * * * \\
\text { Q0WUI8_ARATH); contains Interpro domain(s) } \\
\text { IPR006654 Tryptophan synthase, beta chain }\end{array}$ & 1.07 & 0.96 & 0.89 & 1 & 9.05 & 8.42 & 0.0420321 \\
\hline Solyc01g108480.1.1 & $\begin{array}{l}\text { Serine carboxypeptidase } 1 \text { (AHRD V1 } * * * * \\
\text { B6SW38_MAIZE); contains Interpro domain(s) } \\
\text { IPR001563 Peptidase S10, serine carboxypeptidase }\end{array}$ & 0.59 & 0.96 & 1.63 & 0.93263 & 4.95 & 8.36 & 0.0011708 \\
\hline Solyc08g074630.1.1 & $\begin{array}{l}\text { Polyphenol oxidase (AHRD V1 } * * * \text { _ Q41428_SOLTU); } \\
\text { contains Interpro domain(s) IPR016213 Polyphenol } \\
\text { oxidase, plant }\end{array}$ & 32.58 & 32.89 & 1.01 & 1 & 264.93 & 8.13 & 0.0050633 \\
\hline Solyc03g118970.2.1 & $\begin{array}{l}\text { Multidrug resistance protein mdtK (AHRD V1 } * * * *_{-} \\
\text {MDTK_CITK8); contains Interpro domain(s) IPR015521 } \\
\text { MATE family transporter related protein }\end{array}$ & 12.57 & 11.29 & 0.9 & 0.6652333 & 101.52 & 8.08 & $5.21 \mathrm{E}-31$ \\
\hline
\end{tabular}




\begin{tabular}{|c|c|c|c|c|c|c|c|c|}
\hline Solyc03g098100.2.1 & $\begin{array}{l}\text { Reductase } 2 \text { (AHRD V1 } * * * \text { - Q6TY50_HYDMC); } \\
\text { contains Interpro domain(s) IPR020471 Aldo/keto } \\
\text { reductase subgroup }\end{array}$ & 2.99 & 0.76 & 0.25 & 1 & 24.16 & 8.08 & 0.0019436 \\
\hline Solyc01g107400.2.1 & $\begin{array}{l}\text { Indole-3-acetic acid-amido synthetase GH3.8 (AHRD V1 } \\
* * * \text { - B6U4E2_MAIZE) }\end{array}$ & 0.47 & 0.12 & 0.26 & 0.9323824 & 3.36 & 7.15 & 0.0034934 \\
\hline Solyc03g116900.2.1 & $\begin{array}{l}\text { Manganese transporter MntH (AHRD V1 } * * \text { ** } \\
\text { D3KPZ0_LISMO); contains Interpro domain(s) } \\
\text { IPR001046 Natural resistance-associated macrophage } \\
\text { protein }\end{array}$ & 25.29 & 28.85 & 1.14 & 1 & 178.6 & 7.06 & $2.46 \mathrm{E}-05$ \\
\hline Solyc02g082450.2.1 & $\begin{array}{l}\text { Auxin efflux carrier family protein (AHRD V1 **** } \\
\text { D7L9F0_ARALY); contains Interpro domain(s) } \\
\text { IPR004776 Auxin efflux carrier }\end{array}$ & 1.03 & 0.82 & 0.79 & 1 & 7.14 & 6.91 & $6.13 \mathrm{E}-05$ \\
\hline Solyc06g073760.2.1 & $\begin{array}{l}\text { Beta-D-glucosidase (AHRD V1 } * * * * \text { O82074_TROMA); } \\
\text { contains Interpro domain(s) IPR001764 Glycoside } \\
\text { hydrolase, family } 3 \text {, N-terminal }\end{array}$ & 0.89 & 0.81 & 0.92 & 1 & 5.61 & 6.3 & 0.0026693 \\
\hline Solyc09g084440.2.1 & $\begin{array}{l}\text { Proteinase inhibitor I (AHRD V1 } * * * \text { _ Q3S484_SOLTU); } \\
\text { contains Interpro domain(s) IPR000864 Proteinase } \\
\text { inhibitor I13, potato inhibitor I }\end{array}$ & 0.73 & 0.89 & 1.22 & 1 & 4.02 & 5.51 & 0.0006214 \\
\hline Solyc02g088090.1.1 & $\begin{array}{l}\text { Calmodulin-like protein (AHRD V1 } *_{-} *_{-} \\
\text {B6TXW9_MAIZE); contains Interpro domain(s) } \\
\text { IPR011992 EF-Hand type }\end{array}$ & 4.99 & 3.19 & 0.64 & 0.9625018 & 25.86 & 5.19 & 0.0002988 \\
\hline Solyc12g010960.1.1 & $\begin{array}{l}\text { Alcohol dehydrogenase zinc-binding domain protein } \\
\text { (AHRD V1 } *_{--} \text {A8H5V5_SHEPA); contains Interpro } \\
\text { domain(s) IPR002085 Alcohol dehydrogenase } \\
\text { superfamily, zinc-containing }\end{array}$ & 1.53 & 1.56 & 1.02 & 1 & 7.71 & 5.05 & 0.0288931 \\
\hline Solyc03g020030.2.1 & $\begin{array}{l}\text { Proteinase inhibitor II (AHRD V1 } * * * *_{-} \\
\text {B3F0C1_TOBAC); contains Interpro domain(s) } \\
\text { IPR003465 Proteinase inhibitor I20, Pin2 }\end{array}$ & 0.94 & 1.42 & 1.51 & 0.9441933 & 4.3 & 4.58 & 0.0001705 \\
\hline Solyc02g082920.2.1 & $\begin{array}{l}\text { Endochitinase (Chitinase) (AHRD V1 } * * * * \\
\text { Q43184_SOLTU); contains Interpro domain(s) } \\
\text { IPR000726 Glycoside hydrolase, family } 19 \text {, catalytic }\end{array}$ & 149.07 & 64.52 & 0.43 & 0.3622328 & 679.99 & 4.56 & $2.58 \mathrm{E}-14$ \\
\hline Solyc07g064600.2.1 & $\begin{array}{l}\text { Endoribonuclease L-PSP family protein (AHRD V1 } * *_{-} * \\
\text { D7LAZ0_ARALY); contains Interpro domain(s) } \\
\text { IPR019897 YjgF-like protein, conserved site }\end{array}$ & 168.92 & 182.07 & 1.08 & 1 & 722.36 & 4.28 & 0.0284856 \\
\hline Solyc07g053230.2.1 & $\begin{array}{l}\text { Myb-related transcription factor (AHRD V1 } *--- \\
\text { B5RHV2_MUSBA); contains Interpro domain(s) } \\
\text { IPR015495 Myb transcription factor }\end{array}$ & 1.36 & 1.38 & 1.01 & 1 & 5.6 & 4.11 & $2.54 \mathrm{E}-06$ \\
\hline Solyc03g005040.1.1 & $\begin{array}{l}\text { Calmodulin-like protein (AHRD V1 ***- } \\
\text { Q0VJ70_DATME); contains Interpro domain(s) } \\
\text { IPR011992 EF-Hand type }\end{array}$ & 1.84 & 1.75 & 0.95 & 1 & 7.55 & 4.1 & 0.0047506 \\
\hline
\end{tabular}




\begin{tabular}{|c|c|c|c|c|c|c|c|c|}
\hline Solyc09g089490.2.1 & $\begin{array}{l}\text { Proteinase inhibitor I (AHRD V1 ***-Q33S492_SOLTU); } \\
\text { contains Interpro domain(s) IPR000864 Proteinase } \\
\text { inhibitor I13, potato inhibitor I }\end{array}$ & 27.38 & 14.73 & 0.54 & 0.863136 & 100.64 & 3.68 & 0.0039088 \\
\hline Solyc08g076970.2.1 & $\begin{array}{l}\text { Acetylornithine deacetylase or succinyl-diaminopimelate } \\
\text { desuccinylase (AHRD V1 **_- D4MFM7_9ENTE); } \\
\text { contains Interpro domain(s) IPR011650 Peptidase M20, } \\
\text { dimerisation }\end{array}$ & 32.32 & 33.96 & 1.05 & 1 & 111.05 & 3.44 & 0.0458934 \\
\hline Solyc09g097810.2.1 & $\begin{array}{l}\text { SAR8.2 protein (AHRD V1 } * * * \text { _ Q947G5_CAPAN); } \\
\text { contains Interpro domain(s) IPR004297 Systemic } \\
\text { acquired resistance protein SAR }\end{array}$ & 0.92 & 0.91 & 0.98 & 1 & 3.12 & 3.38 & 0.0404133 \\
\hline Solyc08g006470.2.1 & $\begin{array}{l}\text { Zinc finger family protein (AHRD V1 } * * *- \\
\text { D7MB67_ARALY); contains Interpro domain(s) } \\
\text { IPR007087 Zinc finger, C2H2-type }\end{array}$ & 3.55 & 2.38 & 0.67 & 1 & 11.78 & 3.32 & 0.0185228 \\
\hline Solyc09g089510.2.1 & $\begin{array}{l}\text { Proteinase inhibitor I (AHRD V1 } * * * \text { - Q3S492_SOLTU); } \\
\text { contains Interpro domain(s) IPR000864 Proteinase } \\
\text { inhibitor I13, potato inhibitor I }\end{array}$ & 162.95 & 99.66 & 0.61 & 0.9585738 & 522.39 & 3.21 & 0.0177765 \\
\hline Solyc12g010980.1.1 & $\begin{array}{l}\text { Acyltransferase-like protein (AHRD V1 } *^{*} *_{--} \\
\text {Q589X7_TOBAC); contains Interpro domain(s) } \\
\text { IPR003480 Transferase }\end{array}$ & 22.02 & 22.69 & 1.03 & 1 & 64.41 & 2.93 & 0.0103028 \\
\hline
\end{tabular}




\begin{tabular}{|c|c|c|c|c|c|c|c|c|}
\hline GO.ID & Term & Annotated & Count & Expected & p-value & q-value & Aspect & Genes \\
\hline GO:0030162 & regulation of proteolysis & 118 & 6 & 0.2 & $4.20 \mathrm{E}-08$ & 7.79E-05 & $\mathrm{P}$ & $\begin{array}{l}\text { Solyc03g020030.2, Solyc03g098720.2, } \\
\text { Solyc03g117860.2, Solyc09g084440.2, } \\
\text { Solyc09g089490.2, Solyc09g089510.2 }\end{array}$ \\
\hline GO:0010466 & $\begin{array}{l}\text { negative regulation of peptidase } \\
\text { activity }\end{array}$ & 64 & 5 & 0.11 & $7.30 \mathrm{E}-08$ & $7.79 \mathrm{E}-05$ & $\mathrm{P}$ & $\begin{array}{l}\text { Solyc03g020030.2, Solyc03g098720.2, } \\
\text { Solyc09g0844440.2, Solyc09g089490.2, } \\
\text { Solyc09g089510.2 }\end{array}$ \\
\hline GO:0010951 & $\begin{array}{l}\text { negative regulation of } \\
\text { endopeptidase activity }\end{array}$ & 64 & 5 & 0.11 & $7.30 \mathrm{E}-08$ & 7.79E-05 & $P$ & $\begin{array}{l}\text { Solyc03g020030.2, Solyc03g098720.2, } \\
\text { Solyc09g0844440.2, Solyc09g089490.2, } \\
\text { Solyc09g089510.2 }\end{array}$ \\
\hline GO:0052547 & regulation of peptidase activity & 64 & 5 & 0.11 & $7.30 \mathrm{E}-08$ & $7.79 \mathrm{E}-05$ & $\mathrm{P}$ & $\begin{array}{l}\text { Solyc03g020030.2, Solyc03g098720.2, } \\
\text { Solyc09g0844440.2, Solyc09g089490.2, } \\
\text { Solyc09g089510.2 }\end{array}$ \\
\hline GO:0052548 & $\begin{array}{l}\text { regulation of endopeptidase } \\
\text { activity }\end{array}$ & 64 & 5 & 0.11 & 7.30E-08 & 7.79E-05 & $\mathrm{P}$ & $\begin{array}{l}\text { Solyc03g020030.2, Solyc03g098720.2, } \\
\text { Solyc09g084440.2, Solyc09g089490.2, } \\
\text { Solyc09g089510.2 }\end{array}$ \\
\hline GO:0051346 & $\begin{array}{l}\text { negative regulation of hydrolase } \\
\text { activity }\end{array}$ & 69 & 5 & 0.12 & $1.10 \mathrm{E}-07$ & $9.78 \mathrm{E}-05$ & $\mathrm{P}$ & $\begin{array}{l}\text { Solyc03g020030.2, Solyc03g098720.2, } \\
\text { Solyc09g0844440.2, Solyc09g089490.2, } \\
\text { Solyc09g089510.2 }\end{array}$ \\
\hline GO:0045861 & $\begin{array}{l}\text { negative regulation of } \\
\text { proteolysis }\end{array}$ & 76 & 5 & 0.13 & $1.80 \mathrm{E}-07$ & $1.37 \mathrm{E}-04$ & $\mathrm{P}$ & $\begin{array}{l}\text { Solyc03g020030.2, Solyc03g098720.2, } \\
\text { Solyc09g0844440.2, Solyc09g089490.2, } \\
\text { Solyc09g089510.2 }\end{array}$ \\
\hline GO:0032269 & $\begin{array}{l}\text { negative regulation of cellular } \\
\text { protein metabolic process }\end{array}$ & 145 & 5 & 0.25 & 4.40E-06 & 2.67E-03 & $\mathrm{P}$ & $\begin{array}{l}\text { Solyc03g020030.2, Solyc03g098720.2, } \\
\text { Solyc09g0844440.2, Solyc09g089490.2, } \\
\text { Solyc09g089510.2 }\end{array}$ \\
\hline GO:0051248 & $\begin{array}{l}\text { negative regulation of protein } \\
\text { metabolic process }\end{array}$ & 146 & 5 & 0.25 & 4.50E-06 & $2.67 \mathrm{E}-03$ & $\mathrm{P}$ & $\begin{array}{l}\text { Solyc03g020030.2, Solyc03g098720.2, } \\
\text { Solyc09g0844440.2, Solyc09g089490.2, } \\
\text { Solyc09g089510.2 }\end{array}$ \\
\hline GO:0051336 & regulation of hydrolase activity & 207 & 5 & 0.36 & $2.40 \mathrm{E}-05$ & $1.28 \mathrm{E}-02$ & $\mathrm{P}$ & $\begin{array}{l}\text { Solyc03g020030.2, Solyc03g098720.2, } \\
\text { Solyc09g084440.2, Solyc09g089490.2, } \\
\text { Solyc09g089510.2 }\end{array}$ \\
\hline GO:0032268 & $\begin{array}{l}\text { regulation of cellular protein } \\
\text { metabolic process }\end{array}$ & 370 & 6 & 0.64 & $3.30 \mathrm{E}-05$ & $1.60 \mathrm{E}-02$ & $\mathrm{P}$ & $\begin{array}{l}\text { Solyc03g020030.2, Solyc03g098720.2, } \\
\text { Solyc03g117860.2, Solyc09g084440.2, } \\
\text { Solyc09g089490.2, Solyc09g089510.2 }\end{array}$ \\
\hline GO:0051246 & $\begin{array}{l}\text { regulation of protein metabolic } \\
\text { process }\end{array}$ & 379 & 6 & 0.65 & $3.70 \mathrm{E}-05$ & $1.64 \mathrm{E}-02$ & $\mathrm{P}$ & $\begin{array}{l}\text { Solyc03g020030.2, Solyc03g098720.2, } \\
\text { Solyc03g117860.2, Solyc09g084440.2, } \\
\text { Solyc09g089490.2, Solyc09g089510.2 }\end{array}$ \\
\hline GO:0043086 & $\begin{array}{l}\text { negative regulation of catalytic } \\
\text { activity }\end{array}$ & 234 & 5 & 0.4 & $4.40 \mathrm{E}-05$ & $1.81 \mathrm{E}-02$ & $\mathrm{P}$ & $\begin{array}{l}\text { Solyc03g020030.2, Solyc03g098720.2, } \\
\text { Solyc09g0844440.2, Solyc09g089490.2, } \\
\text { Solyc09g089510.2 }\end{array}$ \\
\hline
\end{tabular}




\begin{tabular}{|c|c|c|c|c|c|c|c|c|}
\hline GO:0031324 & $\begin{array}{l}\text { negative regulation of cellular } \\
\text { metabolic process }\end{array}$ & 400 & 6 & 0.69 & $5.00 \mathrm{E}-05$ & $1.88 \mathrm{E}-02$ & $\mathrm{P}$ & $\begin{array}{l}\text { Solyc03g020030.2, Solyc03g098720.2, } \\
\text { Solyc08g036620.2, Solyc09g084440.2, } \\
\text { Solyc09g089490.2, Solyc09g089510.2 }\end{array}$ \\
\hline GO:0044092 & $\begin{array}{l}\text { negative regulation of molecular } \\
\text { function }\end{array}$ & 243 & 5 & 0.42 & $5.30 \mathrm{E}-05$ & $1.88 \mathrm{E}-02$ & $\mathrm{P}$ & $\begin{array}{l}\text { Solyc03g020030.2, Solyc03g098720.2, } \\
\text { Solyc09g0844440.2, Solyc09g089490.2, } \\
\text { Solyc09g089510.2 }\end{array}$ \\
\hline GO:0009611 & response to wounding & 148 & 4 & 0.25 & 0.00011 & $3.67 \mathrm{E}-02$ & $\mathrm{P}$ & $\begin{array}{l}\text { Solyc08g036620.2, Solyc09g084440.2, } \\
\text { Solyc09g089490.2, Solyc09g089510.2 }\end{array}$ \\
\hline GO:0004866 & endopeptidase inhibitor activity & 67 & 5 & 0.13 & $1.60 \mathrm{E}-07$ & $1.10 \mathrm{E}-04$ & $\mathrm{~F}$ & $\begin{array}{l}\text { Solyc03g020030.2, Solyc03g098720.2, } \\
\text { Solyc09g0844440.2, Solyc09g089490.2, } \\
\text { Solyc09g089510.2 }\end{array}$ \\
\hline GO:0061135 & endopeptidase regulator activity & 67 & 5 & 0.13 & $1.60 \mathrm{E}-07$ & $1.10 \mathrm{E}-04$ & $\mathrm{~F}$ & $\begin{array}{l}\text { Solyc03g020030.2, Solyc03g098720.2, } \\
\text { Solyc09g0844440.2, Solyc09g089490.2, } \\
\text { Solyc09g089510.2 }\end{array}$ \\
\hline GO:0030414 & peptidase inhibitor activity & 68 & 5 & 0.13 & $1.70 \mathrm{E}-07$ & $1.10 \mathrm{E}-04$ & $\mathrm{~F}$ & $\begin{array}{l}\text { Solyc03g020030.2, Solyc03g098720.2, } \\
\text { Solyc09g0844440.2, Solyc09g089490.2, } \\
\text { Solyc09g089510.2 }\end{array}$ \\
\hline GO:0061134 & peptidase regulator activity & 68 & 5 & 0.13 & $1.70 \mathrm{E}-07$ & $1.10 \mathrm{E}-04$ & $\mathrm{~F}$ & $\begin{array}{l}\text { Solyc03g020030.2, Solyc03g098720.2, } \\
\text { Solyc09g0844440.2, Solyc09g089490.2, } \\
\text { Solyc09g089510.2 }\end{array}$ \\
\hline GO:0004867 & $\begin{array}{l}\text { serine-type endopeptidase } \\
\text { inhibitor activity }\end{array}$ & 42 & 4 & 0.08 & $1.10 \mathrm{E}-06$ & $5.70 \mathrm{E}-04$ & $\mathrm{~F}$ & $\begin{array}{l}\text { Solyc03g020030.2, Solyc09g084440.2, } \\
\text { Solyc09g089490.2, Solyc09g089510.2 }\end{array}$ \\
\hline GO:0004857 & enzyme inhibitor activity & 224 & 5 & 0.42 & $6.00 \mathrm{E}-05$ & $2.59 \mathrm{E}-02$ & $\mathrm{~F}$ & $\begin{array}{l}\text { Solyc03g020030.2, Solyc03g098720.2, } \\
\text { Solyc09g0844440.2, Solyc09g089490.2, } \\
\text { Solyc09g089510.2 }\end{array}$ \\
\hline
\end{tabular}




\begin{tabular}{|c|c|c|}
\hline Name & Sequence & Purpose \\
\hline FLS3_pJLSMART/F & ATTCCAAGCTTGGGGCCCATGGAGAAACACATTTTCTTATTG & $\begin{array}{l}\text { Forward primer for cloning chimeric constructs with the } \\
\text { FLS3 LRR domain into pJLSMART }\end{array}$ \\
\hline FLS3_pJLSMART/R & AATTCGGATCCGCCCGGGTATTTACTTCTATGTTTCCAAATG & $\begin{array}{l}\text { Reverse primer for cloning chimeric constructs with the } \\
\text { FLS3 KD domain into pJLSMART }\end{array}$ \\
\hline FLS2_pJLSMART /F & ATTCCAAGCTTGGGGCCCATGATGATGTTAAAGACAGTTG & $\begin{array}{l}\text { Forward primer for cloning chimeric constructs with the } \\
\text { FLS2 LRR domain into pJLSMART }\end{array}$ \\
\hline FLS2_pJLSMART /R & AATTCGGATCCGCCCGGGTATCTTTTACCAAATGAGAAG & $\begin{array}{l}\text { Reverse primer for cloning chimeric constructs with the } \\
\text { FLS2 KD domain into pJLSMART }\end{array}$ \\
\hline $\mathrm{X}-3-2 \_1-2 / \mathrm{R} \_2$ & GTTTTCCGGACGGAAATTAAGTTGCTCGTTGAATCTCATGATAAG & \multirow{2}{*}{$\begin{array}{l}\text { To clone chimeric construct 3-3-2 using overlap } \\
\text { extension PCR }\end{array}$} \\
\hline $\mathrm{X}-3-2 \_3 / \mathrm{F} \_2$ & GATTTCTTATCATGAGATTCAACGAGCAACTTAATTTCCGTCCGG & \\
\hline 3-2-X_1/R_2 & GTTGAATACACCCCCCTTAGGTATTTCACCTTCTAAAACATTAAACGAG & \multirow{2}{*}{$\begin{array}{l}\text { To clone chimeric construct 3-2-2 using overlap } \\
\text { extension PCR }\end{array}$} \\
\hline 3-2-X_2/F_2 & CTCGTTTAATGTTTTAGAAGGTGAAATACCTAAGGGGGGTGTATTCAAC & \\
\hline $\mathrm{X}-2-3 \_2 / \mathrm{R} \_2$ & CAAATTGGATTCATCAAAATTGTTATTGGTAGCATGTTCCAAATCC & \multirow{2}{*}{$\begin{array}{l}\text { To clone chimeric construct 2-2-3 using overlap } \\
\text { extension PCR }\end{array}$} \\
\hline $\mathrm{X}-2-3 \_3 / \mathrm{F} \_2$ & GGATTTGGAACATGCTACCAATAACAATTTTGATGAATCCAATTTG & \\
\hline $\mathrm{X}-3-3 \_3 / \mathrm{F}$ & GGATTCATCAAAATTGTTAGTTGCTCGTTGAATCTC & \multirow{2}{*}{$\begin{array}{l}\text { To clone chimeric constructs } 3-3-3 \text { and } 2-3-3 \text { using } \\
\text { overlap extension PCR }\end{array}$} \\
\hline $\mathrm{X}-3-3 \_1-2 / \mathrm{R}$ & GGATTCATCAAAATTGTTAGTTGCTCGTTGAATCTC & \\
\hline FLS2-iJM_F & ATTCCAAGCTTGGGGCCCGTGAATGACACGGAG & \multirow{2}{*}{ To clone 2JM-3KD } \\
\hline FLS3-KD_pJLSMART /R & AATTCGGATCCGCCCGGGCTAATTTACTTCTATGTTTCC & \\
\hline FLS3-iJM_F & AAAGGGAAGTCCAAAGATGTTGAAAAGGTTCCGG & \multirow[b]{2}{*}{ To clone 3JM-2KD } \\
\hline $\begin{array}{l}\text { FLS2.1-KD_pJLSMART } \\
\text { /R }\end{array}$ & AATTCGGATCCGCCCGGGTTAATCTTTTACCAAATGAGAAG & \\
\hline FLS3-G861S_F & GGGAGGTTCTagcTCTGTGTACA & \multirow{2}{*}{ To mutagenize pJLSMART::3JMKD to 3JMKD(G861S) } \\
\hline FLS3-G861S_R & ACGCCAATCAAATTGGATTC & \\
\hline FLS2-S939A_F & CGCTTGGGAAgccAAGAAGCTAAG & \multirow{2}{*}{ To mutagenize pJLSMART::2JMKD to 2JMKD(S939A) } \\
\hline FLS2-S939A_R & TAACCTAGCACCTTAACTAG & \\
\hline FLS3-G858S_F & GAT TGG CGT GTC AGG TTC TGG CT & \multirow{2}{*}{ To mutagenize pJLSMART::3JMKD to 3JMKD(G858S) } \\
\hline FLS3-G858S_R & AAA TTG GAT TCA TCA AAA TTG TTA GTT G & \\
\hline FLS2-S884G_F & CAGCAGTTTAggtACTGTGTACAAAG & \multirow{2}{*}{ To mutagenize pJLSMART::2JMKD to 2JMKD(S884G) } \\
\hline FLS2-S884G_R & GCTCCAATAATGTTTTCCG & \\
\hline FLS3-G858S/G861S_F & tctagcTCTGTGTACAAAGCCACATTAG & \multirow{2}{*}{$\begin{array}{l}\text { To mutagenize pJLSMART::3JMKD to } \\
\text { 3JMKD(G858S/G861S) and pENTR/D-TOPO::FLS3 to } \\
\text { FLS3(G858S/G861S) }\end{array}$} \\
\hline FLS3-G858S/G861S_R & acctgaCACGCCAATCAAATTGGATTC & \\
\hline FLS3-S914A_F & TACGTGTTCTgctGAACACATAAGAGC & \multirow{2}{*}{ To mutagenize pJLSMART::3JMKD to 3JMKD(S914A) } \\
\hline FLS3-S914A_R & GTGATCACCGAAACAAGG & \\
\hline FLS2-S881G_F & TATTGGAGCCGGCAGTTTAAGTA & \multirow{2}{*}{ To mutagenize pJLSMART::2JMKD to $2 \mathrm{JMKD}(\mathrm{S} 881 \mathrm{G})$} \\
\hline FLS2-S881G_R & ATGTTTTCCGGACGGAAATTATTG & \\
\hline FLS2-K900Q_F & TGTAGCAGTTCAGAAGCTGAATC & \multirow{2}{*}{$\begin{array}{l}\text { To mutagenize pJLSMART::2JMKD to } 2 \mathrm{JMKD}(\mathrm{K} 900 \mathrm{Q}) \\
\text { and pENTR/D-TOPO::FLS2 to FLS2(K900Q) }\end{array}$} \\
\hline FLS2-K900Q_R & ATCTTCCCATCTTCCAGTG & \\
\hline
\end{tabular}




\begin{tabular}{|c|c|c|}
\hline FLS3-G858S_F & GATTGGCGTGTCAGGTTCTGGCT & \multirow{2}{*}{ To mutagenize pJLSMART::3JMKD to 3JMKD(G858S) } \\
\hline FLS3-G858S_R & AAATTGGATTCATCAAAATTGTTAGTTG & \\
\hline FLS3-K877Q_F & GGTTGCAATTCAGGTACTGGATTTG & \multirow{2}{*}{ To mutagenize pJLSMART::3JMKD to 3JMKD(K877Q) } \\
\hline FLS3-K877Q_R & ACAATTCCACTAGCTAATG & \\
\hline FLS2-S881G/S884G_F & ttaggtACTGTGTACAAAGGAACAC & \multirow{2}{*}{$\begin{array}{l}\text { To mutagenize pJLSMART::2JMKD to } \\
\text { 2JMKD(S881G/S884G) and pENTR/D-TOPO::FLS2 to } \\
\text { FLS2(S881G/S884G) }\end{array}$} \\
\hline FLS2-S881G/S884G_R & actgccGGCTCCAATAATGTTTTC & \\
\hline FLS2.1-1 & $\begin{array}{l}\text { TCAAGCGAACCAGTAGGCTTGGTGAAATTCCTGCCGAGCTGTTTTAGAGC } \\
\text { TAGAAATAGC }\end{array}$ & To clone the $\Delta$ FLS2.1 CRISPR/Cas9 construct \\
\hline BothFLS2-1 & $\begin{array}{l}\text { TCAAGCGAACCAGTAGGCTTGTCAGCATTCCAATGGAGGTGTTTTAGAGC } \\
\text { TAGAAATAGC }\end{array}$ & To clone the $\Delta \mathrm{FLS} 2.1 / 2.2 \mathrm{CRISPR} / \mathrm{Cas} 9$ construct \\
\hline FLS3-1 & $\begin{array}{l}\text { TCAAGCGAACCAGTAGGCTTGACTAGAATCACTCGTAACAGTTTTAGAGC } \\
\text { TAGAAATAGC }\end{array}$ & To clone the $\Delta$ FLS3 CRISPR/Cas9 construct \\
\hline BHLH_F & AAGTTCACCATGCAAGAGGT & \multirow{2}{*}{$\begin{array}{l}\text { qRT-PCR primer set for Solyc03g114230.1, BHLH } \\
\text { transcription factor. }\end{array}$} \\
\hline BHLH_R & ATGCATCAACCCCTAATCTCG & \\
\hline UDP_1F & GCAGAACAGGTCAAAGAAATAGC & \multirow{2}{*}{$\begin{array}{l}\text { qRT-PCR primer set for Solyc09g098080.2.1, UDP- } \\
\text { glucosyltransferase }\end{array}$} \\
\hline UDP_1R & GGCAAAACTTCTTCATAGTCGC & \\
\hline MtU6252F & GCATCCCAGTAGGTGAAAGTCGAG & \multirow{2}{*}{$\begin{array}{l}\text { To confirm the loss of the plasmid in CRISPR/Cas9 } \\
\text { plants }\end{array}$} \\
\hline p201R & CGCGCCGAATTCTAGTGATCG & \\
\hline Cas9F7 & GGGTCTCCCGAAGATAATGAGC & \multirow{2}{*}{$\begin{array}{l}\text { To confirm the loss of the Cas9 transgene in } \\
\text { CRISPR/Cas9 plants }\end{array}$} \\
\hline nosT-rev2 & TGATAATCATCGCAAGACC & \\
\hline NPT II F & GGTGGAGAGGCTATTCGGCTATGA & \multirow{2}{*}{$\begin{array}{l}\text { To confirm the loss of the nptII transgene in } \\
\text { CRISPR/Cas9 plants }\end{array}$} \\
\hline NPT II R & CCACAGTCGATGAATCCAGAAAAG & \\
\hline FLS3_-200F & GCAAGTTGACTGACTTTGTAGTCATAC & \multirow{2}{*}{ To Sanger sequence FLS3 in CRISPR/Cas9 knockouts } \\
\hline FLS3_552R & CACCTGTAATGGTATTGTTCCTGAG & \\
\hline S1FLS2_526_F & GGCTTCAACAACAACAATTTCA & \multirow{2}{*}{ To Sanger sequence FLS2.1 in CRISPR/Cas9 knockouts } \\
\hline FLS2.1_1113R & GTTATAGTTGATGGGATCTCCCCGGA & \\
\hline S1FLS2.2_526_F & CTCATCAACAACAGCCTCACA & \multirow{2}{*}{ To Sanger sequence FLS2.2 in CRISPR/Cas9 knockouts } \\
\hline SIFLS2.2_1034_R & TGG AGG GTA AGC ACT TGT AGT GAC A & \\
\hline EF1-a For & GGTGGTTTTGAAGCTGGTATCTCC & \multirow{2}{*}{$\begin{array}{l}\text { To amplify EF1 } \alpha \text { as a positive genomic DNA control in } \\
\text { transgene PCRs }\end{array}$} \\
\hline EF1-a Rev & CCAGTAGGGCCAAAGGTCACA & \\
\hline FLS2.1/2.2-off_target/F & GGC GAA ATG TGG AGA GGG & \multirow{2}{*}{$\begin{array}{l}\text { To amplify a potential off-target region in the } \\
\Delta \text { FLS2.1/2.2 CRISPR/Cas9 plant }\end{array}$} \\
\hline FLS2.1/2.2-off_target/F & GGG TAT GTC TCC AAT TAT GTT GTT GTA AGA C & \\
\hline
\end{tabular}




\begin{tabular}{|c|c|c|}
\hline Name & Reference & Comments \\
\hline \multicolumn{3}{|c|}{ Cloning templates and Gateway entry vectors } \\
\hline pENTR/D-TOPO::FLS3 & (Hind et al., 2016) & DNA template and entry vector for Gateway cloning \\
\hline pENTR/D-TOPO::FLS2 & (Hind et al., 2016) & DNA template and entry vector for Gateway cloning \\
\hline pJLSMART::3-3-3 & This study & $\begin{array}{l}\text { Overlap extension PCR using pENTR/D-TOPO::FLS3 as the DNA template and followed by In- } \\
\text { Fusion cloning at SmaI site }\end{array}$ \\
\hline pJLSMART::2-2-2 & This study & $\begin{array}{l}\text { Overlap extension PCR using pENTR/D-TOPO::FLS2 as DNA template and followed by In- } \\
\text { Fusion cloning at SmaI site }\end{array}$ \\
\hline pJLSMART::3-3-2 & This study & $\begin{array}{l}\text { Overlap extension PCR using pENTR/D-TOPO::FLS2 and pENTR/D-TOPO::FLS3 as DNA } \\
\text { templates and followed by In-Fusion cloning at SmaI site }\end{array}$ \\
\hline pJLSMART::3-2-2 & This study & $\begin{array}{l}\text { Overlap extension PCR using pENTR/D-TOPO::FLS2 and pENTR/D-TOPO::FLS3 as DNA } \\
\text { templates and followed by In-Fusion cloning at SmaI site }\end{array}$ \\
\hline pJLSMART::2-2-3 & This study & $\begin{array}{l}\text { Overlap extension PCR using pENTR/D-TOPO::FLS2 and pENTR/D-TOPO::FLS3 as DNA } \\
\text { templates and followed by In-Fusion cloning at SmaI site }\end{array}$ \\
\hline pJLSMART::2-3-3 & This study & $\begin{array}{l}\text { Overlap extension PCR using pENTR/D-TOPO::FLS2 and pENTR/D-TOPO::FLS3 as DNA } \\
\text { templates and followed by In-Fusion cloning at SmaI site }\end{array}$ \\
\hline $\begin{array}{l}\text { pENTR/D- } \\
\text { TOPO::FLS3(G858S/G861S) }\end{array}$ & This study & Site-directed mutagenesis of pENTR/D-TOPO::FLS3 \\
\hline pENTR/D-TOPO::FLS3(T846A) & This study & Site-directed mutagenesis of pENTR/D-TOPO::FLS3 \\
\hline pENTR/D-TOPO::FLS2(K900Q) & This study & Site-directed mutagenesis of pENTR/D-TOPO::FLS2 \\
\hline $\begin{array}{l}\text { pENTR/D- } \\
\text { TOPO::FLS2(S881G/S884G) }\end{array}$ & This study & Site-directed mutagenesis of pENTR/D-TOPO::FLS2 \\
\hline pENTR/D-TOPO::FLS2(T869A) & This study & Site-directed mutagenesis of pENTR/D-TOPO::FLS2 \\
\hline pJLSMART::3JMKD & This study & PCR using pENTR/D-TOPO::FLS3 as template and followed by In-Fusion cloning at SmaI site \\
\hline pJLSMART::2JMKD & This study & PCR using pENTR/D-TOPO::FLS2 as template and followed by In-Fusion cloning at SmaI site \\
\hline pJLSMART::3JMKD(K877Q) & This study & Site-directed mutagenesis of pJLSMART::3JMKD \\
\hline pJLSMART::2JMKD(K900Q) & This study & Site-directed mutagenesis of pJLSMART::2JMKD \\
\hline pJLSMART::3JMKD(S914A) & This study & Site-directed mutagenesis of pJLSMART::3JMKD \\
\hline pJLSMART::2JMKD(S939A) & This study & Site-directed mutagenesis of pJLSMART::2JMKD \\
\hline pJLSMART::3JMKD(T846A) & This study & Site-directed mutagenesis of pJLSMART::3JMKD \\
\hline pJLSMART::2JMKD(T869A) & This study & Site-directed mutagenesis of pJLSMART::2JMKD \\
\hline pJLSMART::3JMKD(G858S) & This study & Site-directed mutagenesis of pJLSMART::3JMKD \\
\hline pJLSMART::3JMKD(G861S) & This study & Site-directed mutagenesis of pJLSMART::3JMKD \\
\hline pJLSMART::3JMKD(G858S/G861S) & This study & Site-directed mutagenesis of pJLSMART::3JMKD \\
\hline pJLSMART::2JMKD(S881G) & This study & Site-directed mutagenesis of pJLSMART::2JMKD \\
\hline pJLSMART::2JMKD(S884G) & This study & Site-directed mutagenesis of pJLSMART::2JMKD \\
\hline pJLSMART::2JMKD(S881G/S884G) & This study & Site-directed mutagenesis of pJLSMART::2JMKD \\
\hline pJLSMART::3JM-2KD & This study & $\begin{array}{l}\text { Overlap extension PCR using pENTR/D-TOPO::FLS2 and pENTR/D-TOPO::FLS3 as DNA } \\
\text { templates and followed by In-Fusion cloning at SmaI site }\end{array}$ \\
\hline
\end{tabular}




\begin{tabular}{|c|c|c|}
\hline pJLSMART::2JM-3KD & This study & $\begin{array}{l}\text { Overlap extension PCR using pENTR/D-TOPO::FLS2 and pENTR/D-TOPO::FLS3 as DNA } \\
\text { templates and followed by In-Fusion cloning at SmaI site }\end{array}$ \\
\hline pJM51::AvrPtoB $1-359$ & (Cheng et al., 2011) & Entry vector for Gateway cloning \\
\hline pJM51::AvrPtoB ${ }_{1-359}(\mathrm{R} 271 \mathrm{~A} / \mathrm{R} 275 \mathrm{~A})$ & (Cheng et al., 2011) & Entry vector for Gateway cloning \\
\hline \multicolumn{3}{|c|}{ Transient ROS Assays } \\
\hline pGWB417::FLS3 & (Hind et al., 2016) & For expression in plants \\
\hline pGWB417::FLS2 & This study & LR recombination from pENTR/D-TOPO::FLS2 \\
\hline pGWB417::3-3-3 & This study & LR recombination from pJLSMART::3-3-3 \\
\hline pGWB417::2-2-2 & This study & LR recombination from pJLSMART::2-2-2 \\
\hline pGWB417::3-3-2 & This study & LR recombination from pJLSMART::3-3-2 \\
\hline pGWB417::3-2-2 & This study & LR recombination from pJLSMART::3-2-2 \\
\hline pGWB417::2-2-3 & This study & LR recombination from pJLSMART::2-2-3 \\
\hline pGWB417::2-3-3 & This study & LR recombination from pJLSMART::2-3-3 \\
\hline pGWB417::FLS3(K877Q) & (Hind et al., 2016) & For expression in plants \\
\hline pGWB417::FLS3(G858S/G861S) & This study & LR recombination from pJLSMART::FLS3(G858S/G861S) \\
\hline pGWB417::FLS2(K900Q) & This study & LR recombination from pJLSMART::FLS2(K900Q) \\
\hline pGWB417::FLS2(S881G/S884G) & This study & LR recombination from pJLSMART::FLS2(S881G/S884G) \\
\hline pGWB417::FLS2(T869A) & This study & LR recombination from pJLSMART::FLS2(T869A) \\
\hline \multicolumn{3}{|c|}{ In vitro kinase assays } \\
\hline pDEST-HisMBP::3JMKD & This study & LR recombination from pJLSMART::3JMKD \\
\hline pDEST-HisMBP::2JMKD & This study & LR recombination from pJLSMART::2JMKD \\
\hline pDEST-HisMBP::3JMKD(K877Q) & This study & LR recombination from pJLSMART::3JMKD(K877Q) \\
\hline pDEST-HisMBP::2JMKD(K900Q) & This study & LR recombination from pJLSMART::2JMKD(K900Q) \\
\hline pDEST-HisMBP::3JMKD(S914A) & This study & LR recombination from pJLSMART::3JMKD(S914A) \\
\hline pDEST-HisMBP::2JMKD(S939A) & This study & LR recombination from pJLSMART::2JMKD(S939A) \\
\hline pDEST-HisMBP::3JMKD(T846A) & This study & LR recombination from pJLSMART::3JMKD(T846A) \\
\hline pDEST-HisMBP::2JMKD(T869A) & This study & LR recombination from pJLSMART::2JMKD(T869A) \\
\hline pDEST-HisMBP::3JMKD(G858S) & This study & LR recombination from pJLSMART::3JMKD(G858S) \\
\hline pDEST-HisMBP::3JMKD(G861S) & This study & LR recombination from pJLSMART::3JMKD(G861S) \\
\hline $\begin{array}{l}\text { pDEST- } \\
\text { HisMBP::3JMKD(G858S/G861S) }\end{array}$ & This study & LR recombination from pJLSMART::3JMKD(G858S/G861S) \\
\hline pDEST-HisMBP::2JMKD(S884G) & This study & LR recombination from pJLSMART::2JMKD(S884G) \\
\hline $\begin{array}{l}\text { pDEST- } \\
\text { HisMBP::2JMKD(S881G/S884G) }\end{array}$ & This study & LR recombination from pJLSMART::2JMKD(S881G/S884G) \\
\hline pDEST-HisMBP::3JM-2KD & This study & LR recombination from pJLSMART::3JM-2KD \\
\hline pDEST-HisMBP::2JM-3KD & This study & LR recombination from pJLSMART::2JM-3KD \\
\hline \multicolumn{3}{|c|}{ CRISPR-Cas9 } \\
\hline p201N::Cas9 & (Jacobs et al., 2017) & CRISPR/Cas9 cloning vector \\
\hline p201N::Cas9-FLS2.1 & This study & CRISPR/Cas9 knockout vector \\
\hline
\end{tabular}




\begin{tabular}{|c|c|c|}
\hline p201N::Cas9-FLS2.1/FLS2.2 & This study & Gibson cloning for CRISPR/Cas9 knockouts \\
\hline p201N::Cas9-FLS3 & This study & Gibson cloning for CRISPR/Cas9 knockouts \\
\hline \multicolumn{2}{|c|}{ Effector Suppression } & \\
\hline pGWB417::AvrPtoB ${ }_{1-359}$ & this study & LR recombination from pJM51::AvrPtoB1-359 \\
\hline $\begin{array}{l}\text { pGWB417::AvrPtoB } \\
\text { 359(R271A/R275A) }\end{array}$ & This study & LR recombination from pJM51::AvrPtoB1-359(R271A/R275A) \\
\hline pGWB417::YFP & (Roberts et al., 2019) & For expression in plants \\
\hline \multicolumn{2}{|c|}{ Bacterial Strains } & \\
\hline DC3000 $\Delta a v r P t o \Delta a v r P t o B$ & (Lin and Martin, 2005) & \multirow{2}{*}{ Used for bacterial growth curves of CRISPR/Cas9-mediated knockouts } \\
\hline DC3000 $2 a v r P t o \Delta a v r P t o B \Delta f l i C$ & (Kvitko et al., 2009) & \\
\hline DC3000::AvrPtoB ${ }_{1-359}$ & (Cheng et al., 2011) & \multirow{2}{*}{ Used for bacterial growth curves in the effector inhibition assay } \\
\hline DC3000::AvrPtoB ${ }_{1-359}(\mathrm{R} 271 \mathrm{~A} / \mathrm{R} 275 \mathrm{~A})$ & (Cheng et al., 2011) & \\
\hline
\end{tabular}

\section{References}

Cheng, W., Munkvold, K.R., Gao, H., Mathieu, J., Schwizer, S., Wang, S., Yan, Y.-b., Wang, J., Martin, G.B., and Chai, J. (2011). Structural Analysis of Pseudomonas syringae AvrPtoB Bound to Host BAK1 Reveals Two Similar Kinase-Interacting Domains in a Type III Effector. Cell Host \& Microbe 10:616-626.

Hind, S.R., Strickler, S.R., Boyle, P.C., Dunham, D.M., Bao, Z., O'Doherty, I.M., Baccile, J.A., Hoki, J.S., Viox, E.G., Clarke, C.R., et al. (2016). Tomato receptor FLAGELLIN-SENSING 3 binds flgII-28 and activates the plant immune system. Nat Plants 2:16128.

Jacobs, T.B., Zhang, N., Patel, D., and Martin, G.B. (2017). Generation of a collection of mutant tomato lines using pooled CRISPR libraries. Plant Physiol 174:2023-2037.

Kvitko, B.H., Park, D.H., Velasquez, A.C., Wei, C.-F., Russell, A.B., Martin, G.B., Schneider, D.J., and Collmer, A. (2009). Deletions in the repertoire of Pseudomonas syringae pv. tomato DC3000 type III secretion effector genes reveal functional overlap among effectors. PLoS Pathog 5:e1000388.

Lin, N.C., and Martin, G.B. (2005). An avrPto/avrPtoB mutant of Pseudomonas syringae pv. tomato DC3000 does not elicit Pto-mediated resistance and is less virulent on tomato. Mol Plant-Microbe Interact 18:43-51.

Pombo, M.A., Zheng, Y., Fei, Z., Martin, G.B., and Rosli, H.G. (2017). Use of RNA-seq data to identify and validate RT-qPCR reference genes for studying the tomato-Pseudomonas pathosystem. Sci Rep 7:44905.

Roberts, R., Hind, S.R., Pedley, K.F., Diner, B.A., Szarzanowicz, M.J., Luciano-Rosario, D., Majhi, B.B., Popov, G., Sessa, G., Oh, C.S., et al. (2019). Mai1 protein acts between host recognition of pathogen effectors and MAPK signaling. Mol Plant Microbe Interact 32:1496-1507.

Rosli, H.G., Zheng, Y., Pombo, M.A., Zhong, S., Bombarely, A., Fei, Z., Collmer, A., and Martin, G.B. (2013). Transcriptomics-based screen for genes induced by flagellin and repressed by pathogen effectors identifies a cell wall-associated kinase involved in plant immunity. Genome Biol 14:R139. 


\section{Supplemental Methods}

\section{Plant growth conditions, inoculations, and bacterial growth assays}

Tomato seedlings were grown under the conditions described in (Roberts et al., 2019). P. syringae strains were grown on King's B medium and suspended to a final concentration of 2

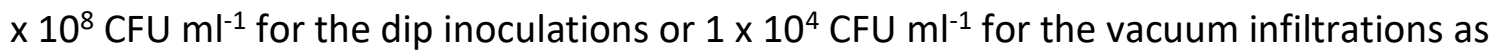
described previously (Roberts et al., 2019), and Silwet-L77 was added to a final concentration of $0.04 \%$. Vacuum infiltrations were performed as previously described (Roberts et al., 2019). For the dip inoculations, three-week-old seedlings were placed in a $100 \%$ relative humidity chamber for 14 hours prior to inoculation, then dipped into the bacterial suspension for 10 seconds. Seedlings were placed back into the humidity chamber for 2 hours after inoculation. Leaflets were then excised from the plants and surface disinfected for two minutes in halfstrength bleach before sampling, and bacterial populations were quantified on Day 0 and Day 2 or Day 3 as described in (Roberts et al., 2019). Experiments were repeated three times ( $n=3$ plants) and shown is a single representative replicate. Data are means of individual plants in a single replicate (represented as points) and horizontal lines are the means of the three plants in a single experiment. Error bars are +/- s.d. and significance was determined using the Prism 8 program (GraphPad Software, https://www.graphpad.com/scientific-software/prism/). For a list of bacterial strains used in this study, see Table S3.

\section{Generation of CRISPR Cas9-mediated knockout lines}

Geneious R9 software ( https://www.geneious.com/) was used to design gRNAs to target Fls3, Fls2.1, or both Fls2.1/Fls2.2 as described previously (Jacobs et al., 2017; Zhang et al., 2020) using the tomato genome version SL2.5 (Tomato Genome Consortium, 2012). The gRNAs selected for each of the genes were: Fls3, TGTTACGAGTGATTCTAGTC; Fls2.1, GAAATTCCTGCCGAGCTGGG; Fls2.1/FIs2.2, TACCTCCATTGGAATGCTGAC. Possible off-targets were predicted using the Geneious software. The gRNAs were cloned into the p201N::Cas9 vector using the Gibson assembly kit (New England Biolabs, www.neb.com), transformed into Agrobacterium tumafaciens strain LBA4404, and transformed into tomato (Rio Grande-prf3) as 
described in (Jacobs et al., 2017; Zhang et al., 2020). To induce mutations in both Fls3 and Fls2.1/2.2 in the same plant ( $\Delta \mathrm{Fls} 2.1 / 2.2 / 3)$, the constructs used to induce the individual mutations were transformed into LBA4404 and the Agrobacterium cultures were mixed 1:1 prior to tomato transformation. Tomato transformations were performed at the Boyce Thompson Institute transformation facility (Gupta and Van Eck, 2016; Van Eck et al., 2019). More information may be found in Table S1-3 and Fig. S1, and on the Plant CRISPR database at plantcrispr.org.

\section{Peptides used in the ROS bioassays}

The flgII-28 (ESTNILQRMRELAVQSRNDSNSSTDRDA), flg22 (QRLSTGSRINSAKDDAAGLQIA), or E. coli flg15 (RINSAKDDAAGLQIA) peptides were commercially ordered from GenScript (https://www.genscript.com/) with >95\% purity. Solubility testing was performed by the company, and all peptides were soluble in water. Peptides were also all predicted soluble using a prediction software (https://pepcalc.com/peptide-solubility-calculator.php). Multiple independent batches of flgll-28, flg22, and flg15 peptides were used throughout this paper, and the same observations were made with all batches. Because it is known that flg22 can be a contaminant in commercial peptides (Mueller et al., 2012), all peptides were confirmed not to be contaminated with flg22 before experimental use by testing them in ROS assays using Rio Grande, $\Delta$ Fls2 plants (which do not respond to flg22 (Jacobs et al., 2017)), and Yellow Pear (which does not respond to flgll-28 (Hind et al., 2016)). New peptide batches were compared side-by-side with old batches in ROS assays to confirm the sensitivity was similar in Rio Grande. We did not observe any instances of contamination or changes in sensitivities between batches of peptides. The extended ROS response was always observed for flgll-28 in tomato and has been observed in previous studies of flgll-28 (Clarke et al., 2013; Hind et al., 2016). The extended ROS response to flgll-28 was observed regardless of peptide concentration (10 nM-1 $\mu \mathrm{M})$. 


\section{Cloning}

The chimeric constructs were generated via overlap extension PCR using pENTR/DTOPO::Fls3 and pENTR/D-TOPO::Fls2 as templates (Hind et al., 2016). The 2KD, 2JMKD, 3KD, and 3JMKD ORFs were amplified from pENTR/D-TOPO::Fls3 or pENTR/D-TOPO::Fls2 using PCR. PCR products were inserted into the entry vector pJLSMART (Mathieu et al., 2014) at the Smal cut site using the In-Fusion cloning kit and following the manufacturer's instructions (Takara, https://www.takarabio.com/). Sequences were confirmed via Sanger sequencing, and then the chimeric construct ORFs were recombined into the Gateway vector pGWB417 (Nakagawa et al., 2009; Nakagawa et al., 2007) using the LR Clonase II following the manufacturer's instructions (Thermo Fisher Scientific, https://www.thermofisher.com/us/en/home.html). Recombination was confirmed using Sanger sequencing.

Mutagenesis of the 2JMKD, 3JMKD, Fls2, and Fls3 clones was performed using the entry vectors and the Q5 Site-Directed Mutagenesis kit following the manufacturer's instructions (New England Biolabs, www.neb.com). After mutations were confirmed using Sanger sequencing, ORFs were recombined into pDEST-HisMBP (2JMKD or 3JMKD) (Nallamsetty et al., 2005) or pGWB417 (Fls2 or Fls3) using the LR Clonase II enzyme. For a full list of primers and constructs used in this study, see Table S2 and S3.

\section{Agroinfiltrations}

Binary vectors harboring Fls3 or Fls2 were transformed into Agrobacterium tumefaciens strain GV3101. Cultures were grown in induction medium for 5 hours at room temperature with shaking before adjusting the concentrations for infiltration. All cultures were prepared to a final $\mathrm{OD}_{600}$ of 0.2 and, the Fls3- and Fls2- containing bacterial cultures were mixed 1:1 with Agrobacterium cultures expressing the $p 19$ viral suppressor of silencing. For Figure 4, the bacterial cultures containing Fls3 or Fls2 and AvrPtoB $_{1-359}$ or AvrPtoB $_{1-359}(R 271 A / R 275 A)$ were mixed in equal parts with the $p 19$ (1:1:1) before infiltration. 


\section{References}

Clarke, C.R., Chinchilla, D., Hind, S.R., Taguchi, F., Miki, R., Ichinose, Y., Martin, G.B., Leman, S., Felix, G., and Vinatzer, B.A. (2013). Allelic variation in two distinct Pseudomonas syringae flagellin epitopes modulates the strength of plant immune responses but not bacterial motility. New Phytol 200:847-860.

Gupta, S., and Van Eck, J. (2016). Modification of plant regeneration medium decreases the time for recovery of Solanum lycopersicum cultivar M82 stable transgenic lines. Plant Cell, Tissue and Organ Culture (PCTOC) 127:417-423.

Hind, S.R., Strickler, S.R., Boyle, P.C., Dunham, D.M., Bao, Z., O'Doherty, I.M., Baccile, J.A., Hoki, J.S., Viox, E.G., Clarke, C.R., et al. (2016). Tomato receptor FLAGELLIN-SENSING 3 binds flgll-28 and activates the plant immune system. Nat Plants 2:16128.

Jacobs, T.B., Zhang, N., Patel, D., and Martin, G.B. (2017). Generation of a collection of mutant tomato lines using pooled CRISPR libraries. Plant Physiol 174:2023-2037.

Mathieu, J., Schwizer, S., and Martin, G.B. (2014). Pto kinase binds two domains of AvrPtoB and its proximity to the effector E3 ligase determines if it evades degradation and activates plant immunity. PLoS Pathog 10:e1004227.

Mueller, K., Chinchilla, D., Albert, M., Jehle, A.K., Kalbacher, H., Boller, T., and Felix, G. (2012). Contamination risks in work with synthetic peptides: flg22 as an example of a pirate in commercial peptide preparations. The Plant cell 24:3193-3197.

Nakagawa, T., Ishiguro, S., and Kimura, T. (2009). Gateway vectors for plant transformation. Plant Biotechnology 26:275-284.

Nakagawa, T., Suzuki, T., Murata, S., Nakamura, S., Hino, T., Maeo, K., Tabata, R., Kawai, T., Tanaka, K., Niwa, Y., et al. (2007). Improved Gateway binary vectors: high-performance vectors for creation of fusion constructs in transgenic analysis of plants. Biosci Biotechnol Biochem 71:2095-2100.

Nallamsetty, S., Austin, B.P., Penrose, K.J., and Waugh, D.S. (2005). Gateway vectors for the production of combinatorially-tagged His6-MBP fusion proteins in the cytoplasm and periplasm of Escherichia coli. Protein Sci 14:2964-2971.

Roberts, R., Mainiero, S., Powell, A.F., Liu, A.E., Shi, K., Hind, S.R., Strickler, S.R., Collmer, A., and Martin, G.B. (2019). Natural variation for unusual host responses and flagellin-mediated immunity against Pseudomonas syringae in genetically diverse tomato accessions. New Phytol 223:447-461.

Tomato Genome Consortium. (2012). The tomato genome sequence provides insights into fleshy fruit evolution. Nature 485:635-641.

Van Eck, J., Keen, P., and Tjahjadi, M. (2019). Agrobacterium tumefaciens-Mediated Transformation of Tomato. Methods Mol Biol 1864:225-234.

Zhang, N., Roberts, H.M., Van Eck, J., and Martin, G.B. (2020). Generation and molecular characterization of CRISPR/Cas9-induced mutations in 63 immunity-associated genes in tomato reveals specificity and a range of gene modifications. Frontiers in Plant Science In Press. 